Geometry \& Topology

Volume 3 (1999) 405-466

Published: 17 December 1999

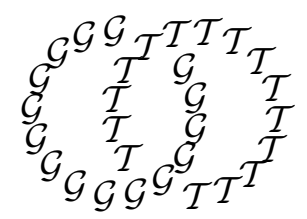

\title{
An elementary approach to the mapping class group of a surface
}

\author{
BRONISLAW WAJNRYB \\ Department of Mathematics \\ Technion, 32000 Haifa, Israel \\ Email: wajnryb@techunix.technion.ac.il
}

\begin{abstract}
We consider an oriented surface $S$ and a cellular complex $X$ of curves on $S$, defined by Hatcher and Thurston in 1980. We prove by elementary means, without Cerf theory, that the complex $X$ is connected and simply connected. From this we derive an explicit simple presentation of the mapping class group of $S$, following the ideas of Hatcher-Thurston and Harer.
\end{abstract}

\section{AMS Classification numbers Primary: 20F05,20F34,57M05}

Secondary: 20F38,57M60

Keywords: Mapping class group, surface, curve complex, group presentation

Proposed: Joan Birman

Seconded: Walter Neumann, Cameron Gordon
Received: 7 January 1999

Revised: 18 November 1999

Copyright Geometry and Topology 


\section{Introduction}

Let $S$ be a compact oriented surface of genus $g \geq 0$ with $n \geq 0$ boundary components and $k$ distinguished points. The mapping class group $\mathcal{M}_{g, n, k}$ of $S$ is the group of the isotopy classes of orientation preserving homeomorphisms of $S$ which keep the boundary of $S$ and all the distinguished points pointwise fixed. In this paper we study the problem of finding a finite presentation for $\mathcal{M}_{g, n}=\mathcal{M}_{g, n, 0}$. We restrict our attention to the case $n=1$. The case $n=0$ is easily obtained from the case $n=1$. In principle the case of $n>1$ (and the case of $k>0$ ) can be obtained from the case $n=1$ via standard exact sequences, but this method does not produce a global formula for the case of several boundary components and the presentation (in contrast to the ones we shall describe for the case $n=0$ and $n=1$ ) becomes rather ugly. On the other hand Gervais in [6] succeeded recently to produce a finite presentation of $\mathcal{M}_{g, n}$ starting from the results in [20] and using a new approach.

A presentation for $g=1$ has been known for a long time. A quite simple presentation for $g=2$ was established in 1973 in [1], but the method did not generalize to higher genus. In $1975 \mathrm{McCool}$ proved in [19], by purely algebraic methods, that $\mathcal{M}_{g, 1}$ is finitely presented for any genus $g$. It seems that extracting an explicit finite presentation from his proof is very difficult. In 1980 appeared the groundbreaking paper of Hatcher and Thurston [9] in which they gave an algorithm for constructing a finite presentation for the group $\mathcal{M}_{g, 1}$ for an arbitrary $g$. In 1981 Harer applied their algorithm in [7] to obtain a finite (but very unwieldy) explicit presentation of $\mathcal{M}_{g, 1}$. His presentation was simplified by Wajnryb in 1983 in [20]. A subsequent Errata [3] corrected small errors in the latter. The importance of the full circle of ideas in these papers can be jugded from a small sample of subsequent work which relied on the presentation in [20], eg [14], [16], [17], [18]. The proof of Hatcher and Thurston was deeply original, and solved an outstanding open problem using novel techniques. These included arguments based upon Morse and Cerf Theory, as presented by Cerf in [4].

In this paper we shall give, in one place, a complete hands-on proof of a simple presentation for the groups $\mathcal{M}_{g, 0}$ and $\mathcal{M}_{g, 1}$. Our approach will follow the lines set in [9], but we will be able to use elementary methods in the proof of the connectivity and simple connectivity of the cut system complex. In particular, our work does not rely on Cerf theory. At the same time we will gather all of the computational details in one place, making the result accesible for independent checks. Our work yields a slightly different set of generators and relations from the ones used in [20] and in [3]. The new presentation makes the computations 
in section 4 a little simpler. We shall give both presentations and prove that they are equivalent.

A consequence of this paper is that, using $\mathrm{Lu}$ [16] or Matveev and Polyak [18], the fundamental theorem of the Kirby calculus [13] now has a completely elementary proof (ie, one which makes no appeal to Cerf theory or high dimensional arguments).

This paper is organised as follows. In section 2 we give a new proof of the main theorem of Hatcher and Thurston. In section 3 we derive a presentation of the mapping class group following (and explaining) the procedure described in [9] and in [7]. In section 4 we reduce the presentation to the simple form of Theorem 1 repeating the argument from [20] with changes required by a slightly different setup. In section 5 we deduce the case of a closed surface and in section 6 we translate the presentation into the form given in [20], see Remark 1 below.

We start with a definition of a basic element of the mapping class group.

Definition 1 A (positive) Dehn twist with respect to a simple closed curve $\alpha$ on an oriented surface $S$ is an isotopy class of a homeomorphism $h$ of $S$, supported in a regular neighbourhood $N$ of $\alpha$ (an annulus), obtained as follows: we cut $S$ open along $\alpha$, we rotate one side of the cut by 360 degrees to the right (this makes sense on an oriented surface) and then glue the surface back together, damping out the rotation to the identity at the boundary of $N$. The Dehn twist (or simply twist) with respect to $\alpha$ will be denoted by $T_{\alpha}$. If curves $\alpha$ and $\beta$ intersect only at one point and are transverse then $T_{\alpha}(\beta)$, up to an isotopy, is obtained from the union $\alpha \cup \beta$ by splitting the union at the intersection point.

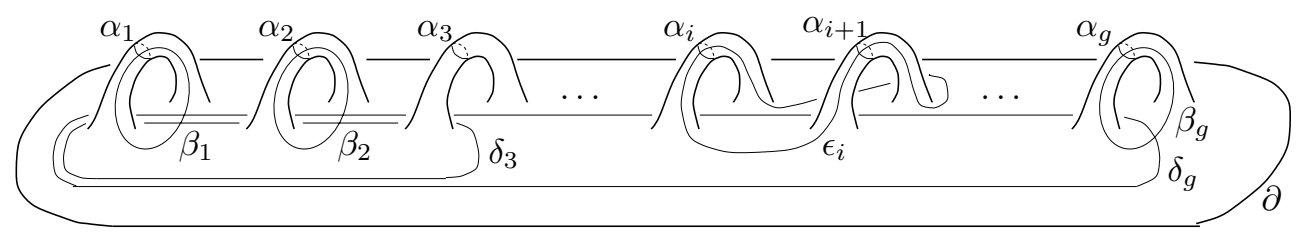

Figure 1: Surface $S_{g, 1}$

We shall say that two elements $a, b$ of a group are braided (or satisfy the braid relation) if $a b a=b a b$.

Theorem 1 Let $S_{g, 1}$ be a compact, orientable surface of genus $g \geq 3$ with one boundary component. Let $a_{i}, b_{i}, e_{i}$ denote Dehn twists about the curves 
$\alpha_{i}, \beta_{i}, \epsilon_{i}$ on $S_{g, 1}$ depicted on Figure 1. The mapping class group $\mathcal{M}_{g, 1}$ of $S_{g, 1}$ is generated by elements $b_{2}, b_{1}, a_{1}, e_{1}, a_{2}, e_{2}, \ldots, a_{g-1}, e_{g-1}, a_{g}$ and has defining relations:

(M1) Elements $b_{2}$ and $a_{2}$ are braided and $b_{2}$ commutes with $b_{1}$. Every other pair of consecutive elements (in the above order) is braided and every other pair of non-consecutive elements commute.

$$
\left(b_{1} a_{1} e_{1} a_{2}\right)^{5}=b_{2} a_{2} e_{1} a_{1} b_{1}^{2} a_{1} e_{1} a_{2} b_{2}
$$

(M3) $\quad d_{3} a_{1} a_{2} a_{3}=d_{1,2} d_{1,3} d_{2,3}$, where

$d_{1,2}=\left(a_{2} e_{1} a_{1} b_{1}\right)^{-1} b_{2}\left(a_{2} e_{1} a_{1} b_{1}\right), \quad d_{1,3}=t_{2} d_{1,2} t_{2}^{-1}, \quad d_{2,3}=t_{1} d_{1,3} t_{1}^{-1}$,

$t_{1}=e_{1} a_{1} a_{2} e_{1}, \quad t_{2}=e_{2} a_{2} a_{3} e_{2}, \quad d_{3}=b_{2} a_{2} e_{1} b_{1}^{-1} d_{1,3} b_{1} e_{1}^{-1} a_{2}^{-1} b_{2}^{-1}$.

Theorem 2 The mapping class group $\mathcal{M}_{2,1}$ of an orientable surface $S_{2,1}$ of genus $g=2$ with one boundary component is generated by elements $b_{2}, b_{1}, a_{1}$, $e_{1}, a_{2}$ and has defining relations (M1) and (M2).

Theorem 3 The mapping class group $\mathcal{M}_{g, 0}$ of a compact, closed, orientable surface of genus $g>1$ can be obtained from the above presentation of $\mathcal{M}_{g, 1}$ by adding one more relation:

$$
\left[b_{1} a_{1} e_{1} a_{2} \ldots a_{g-1} e_{g-1} a_{g} a_{g} e_{g-1} a_{g-1} \ldots e_{1} a_{1} b_{1}, d_{g}\right]=1 \text {, where }
$$

$t_{i}=e_{i} a_{i} a_{i+1} e_{i}$, for $i=1,2, \ldots, g-1, d_{2}=d_{1,2}$,

$d_{i}=\left(b_{2} a_{2} e_{1} b_{1}^{-1} t_{2} t_{3} \ldots t_{i-1}\right) d_{i-1}\left(b_{2} a_{2} e_{1} b_{1}^{-1} t_{2} t_{3} \ldots t_{i-1}\right)^{-1}$, for $i=3,4, \ldots, g$

The presentations in Theorems 1 and 3 are equivalent to but not the same as those in [20] and [3]. We now give alternative presentations of Theorems 1 and 3 , with the goal of correlating the work in this paper with that in [20] and [3]. See Remark 1, below, for a dictionary which allows one to move between Theorems $1^{\prime}$ and $3^{\prime}$ and the results in [20] and [3]. See Section 6 of this paper for a proof that the presentations in Theorems 1 and $1^{\prime}$, and also Theorems 3 and $3^{\prime}$, are equivalent.

Theorem $\mathbf{1}^{\prime}$ The mapping class group $\mathcal{M}_{g, 1}$ admits a presentation with generators $b_{2}, b_{1}, a_{1}, e_{1}, a_{2}, e_{2}, \ldots, a_{g-1}, e_{g-1}, a_{g}$ and with defining relations:

(A) Elements $b_{2}$ and $a_{2}$ are braided and $b_{2}$ commutes with $b_{1}$. Every other pair of consecutive elements (in the above order) is braided and every other pair of non-consecutive elements commute.

$$
\left(b_{1} a_{1} e_{1}\right)^{4}=\left(a_{2} e_{1} a_{1} b_{1}^{2} a_{1} e_{1} a_{2}\right) b_{2}\left(a_{2} e_{1} a_{1} b_{1}^{2} a_{1} e_{1} a_{2}\right)^{-1} b_{2}
$$

Geometry and Topology, Volume 3 (1999) 
(C) $\quad e_{2} e_{1} b_{1} \tilde{d}_{3}=\tilde{t}_{1}^{-1} \tilde{t}_{2}^{-1} b_{2} \tilde{t}_{2} \tilde{t}_{1} \tilde{t}_{2}^{-1} b_{2} \tilde{t}_{2} b_{2} \quad$ where

$\tilde{t}_{1}=a_{1} b_{1} e_{1} a_{1}, \quad \tilde{t}_{2}=a_{2} e_{1} e_{2} a_{2}, \quad \tilde{d}_{3}=\left(a_{3} e_{2} a_{2} e_{1} a_{1} u\right) v\left(a_{3} e_{2} a_{2} e_{1} a_{1} u\right)^{-1}$, $u=e_{2}^{-1} a_{3}^{-1} \tilde{t}_{2}^{-1} b_{2} \tilde{t}_{2} a_{3} e_{2}, \quad v=\left(a_{2} e_{1} a_{1} b_{1}\right)^{-1} b_{2}\left(a_{2} e_{1} a_{1} b_{1}\right)$.

Theorem $3^{\prime}$ The mapping class group $\mathcal{M}_{g, 0}$ of a compact, closed, orientable surface of genus $g>1$ can be obtained from the above presentation of $\mathcal{M}_{g, 1}$ by adding one more relation:

(D) $\left[a_{g} e_{g-1} a_{g-1} \ldots e_{1} a_{1} b_{1}^{2} a_{1} e_{1} \ldots a_{g-1} e_{g-1} a_{g}, \tilde{d}_{g}\right]=1$, where

$\tilde{d}_{g}=u_{g-1} u_{g-2} \ldots u_{1} b_{1}\left(u_{g-1} u_{g-2} \ldots u_{1}\right)^{-1}, \quad u_{1}=\left(b_{1} a_{1} e_{1} a_{2}\right)^{-1} v_{1} a_{2} e_{1} a_{1}$,

$u_{i}=\left(e_{i-1} a_{i} e_{i} a_{i+1}\right)^{-1} v_{i} a_{i+1} e_{i} a_{i}$ for $i=2, \ldots, g-1$,

$v_{1}=\left(a_{2} e_{1} a_{1} b_{1}^{2} a_{1} e_{1} a_{2}\right) b_{2}\left(a_{2} e_{1} a_{1} b_{1}^{2} a_{1} e_{1} a_{2}\right)^{-1}$,

$v_{i}=\tilde{t}_{i-1}^{-1} \tilde{t}_{i}^{-1} v_{i-1} \tilde{t}_{i} \tilde{t}_{i-1} \quad$ for $\quad i=2, \ldots, g-1$,

$\tilde{t}_{1}=a_{1} e_{1} b_{1} a_{1}, \quad \tilde{t}_{i}=a_{i} e_{i} e_{i-1} a_{i} \quad$ for $\quad i=2, \ldots, g-1$.

Remark 1 We now explain how to move back and forth between the results in this paper and those in [20] and [3].

(i) The surface and the curves in [20] look different from the surface and the curves on Figure 1. However if we compare the Dehn twist generators in Theorem 1' with those in Theorem 1 of [20] and [3] we see that corresponding curves have the same intersection pattern. Thus there exists a homeomorphism of one surface onto the other which takes the curves of one family onto the corresponding curves of the other family. The precise correspondence is given by:

$$
\longleftrightarrow \begin{aligned}
& \left(b_{2}, b_{1}, a_{1}, e_{1}, a_{2}, e_{2}, \ldots, a_{g-1}, e_{g-1}, a_{g}\right) \\
& \left(d, a_{1}, b_{1}, a_{2}, b_{2}, a_{3}, \ldots, b_{n-1}, a_{n}, b_{n}\right),
\end{aligned}
$$

where the top sequence refers to Dehn twists about the curves in Figure 1 of this paper and the bottom sequence refers to Dehn twists about the curves in Figure 1 on page 158 of [20].

(ii) Composition of homeomorphisms in [20] was performed from left to right, while in the present paper we use the standard composition from right to left.

(iii) The element $d_{g}$ in this paper represents a Dehn twist about the curve $\delta_{g}$ in Figure 1 of this paper. The element $\tilde{d}_{g}$ in relation (D) of Theorem $3^{\prime}$ represents a Dehn twist about the curve $\beta_{g}$ in Figure 1 . We wrote $d_{g}$ as a particular product of the generators in $\mathcal{M}_{g, 1}$. It follows from the argument in the last section that any other such product representing $d_{g}$ will also do. 
(iv) In the case of genus $g=2$ we should omit relation (C) in Theorems $1^{\prime}$ and $3^{\prime}$.

Here is the plan of the proof of the theorems. Following Hatcher and Thurston we define a 2-dimensional cell complex $X$ on which the mapping class group $\mathcal{M}_{g, 1}$ acts by cellular transformations and the action is transitive on the vertices of $X$. We give a new elementary proof of the fact that $X$ is connected and simply connected. We then describe the stabilizer $H$ of one vertex of $X$ under the action of $\mathcal{M}_{g, 1}$ and we determine an explicit presentation of $H$. Following the algorithm of Hatcher and Thurston we get from it a presentation of $\mathcal{M}_{g, 1}$. Finally we reduce the presentation to the form in Theorem 1 and as a corollary get Theorem 3.

Acknowledgements I wish to thank very much the referee, who studied the paper very carefully and made important suggestions to improve it.

This research was partially supported by the Fund for the Promotion of Research at the Technion and the Dent Charitable Trust - a non military research fund.

\section{Cut-system complex}

We denote by $S$ a compact, connected oriented surface of genus $g>0$ with $n \geq 0$ boundary components. We denote by $\bar{S}$ a closed surface obtained from $S$ by capping each boundary component with a disk. By a curve we shall mean a simple closed curve on $S$. We are mainly interested in the isotopy classes of curves on $S$. The main goal in the proofs will be to decrease the number of intersection points between different curves. If the Euler characteristic of $S$ is negative we can put a hyperbolic metric on $S$ for which the boundary curves are geodesics. Then the isotopy class of any non-separating curve on $S$ contains a unique simple closed geodesic, which is the shortest curve in its isotopy class. If we replace each non-separating curve by the unique geodesic isotopic to it we shall minimize the number of intersection points between every two non-isotopic curves, by Corollary 5.1. So we can think of curves as geodesics. In the proof we may construct new curves, which are not geodesics and which have small intersection number with some other curves. When we replace the new curve by the corresponding geodesic we further decrease the intersection number. If $S$ is a closed torus we can choose a flat metric on $S$. Now geodesics are not unique but still any choice of geodesics will minimize the intersection number 
of any pair of non-isotopic curves. If two curves are isotopic on $S$ then they correspond to the same geodesic, but we shall call them disjoint because we can isotop one off the other. Geodesics are never tangent.

If $\alpha$ and $\beta$ are curves then $|\alpha \cap \beta|$ denotes their geometric intersection number, ie, the number of intersection points of $\alpha$ and $\beta$. If $\alpha$ is a curve we denote by $[\alpha]$ the homology class represented by $\alpha$ in $H_{1}(\bar{S}, \mathbb{Z})$, up to a sign. We denote by $i(\alpha, \beta)$ the absolute value of the algebraic intersection number of $\alpha$ and $\beta$. It depends only on the classes $[\alpha]$ and $[\beta]$.

We shall describe now the cut-system complex $X$ of $S$. To construct $X$ we consider collections of $g$ disjoint curves $\gamma_{1}, \gamma_{2}, \ldots, \gamma_{g}$ in $S$ such that when we cut $S$ open along these curves we get a connected surface (a sphere with $2 g+n$ holes). An isotopy class of such a collection we call a cut system $\left\langle\gamma_{1}, \ldots, \gamma_{g}\right\rangle$. We can say that a cut system is a collection of geodesics. A curve is contained in a cut-system if it is one of the curves of the collection. If $\gamma_{i}^{\prime}$ is a curve in $S$, which meets $\gamma_{i}$ at one point and is disjoint from other curves $\gamma_{k}$ of the cut system $\left\langle\gamma_{1}, \ldots, \gamma_{g}\right\rangle$, then $\left\langle\gamma_{1}, \ldots, \gamma_{i-1}, \gamma_{i}^{\prime}, \gamma_{i+1}, \ldots, \gamma_{g}\right\rangle$ forms another cut system. In such a situation the replacement $\left\langle\gamma_{1}, \ldots, \gamma_{i}, \ldots, \gamma_{g}\right\rangle \rightarrow\left\langle\gamma_{1}, \ldots, \gamma_{i}^{\prime}, \ldots, \gamma_{g}\right\rangle$ is called a simple move. For brewity we shall often drop the symbols for unchanging circles and shall write $\left\langle\gamma_{i}\right\rangle \rightarrow\left\langle\gamma_{i}^{\prime}\right\rangle$. The cut systems on $S$ form the 0 -skeleton (the vertices) of the complex $X$. We join two vertices by an edge if and only if the corresponding cut systems are related by a simple move. We get the 1 -skeleton $X^{1}$. By a path we mean an edge-path in $X^{1}$. It consists of a sequence of vertices $\mathbf{p}=\left(v_{1}, v_{2}, \ldots, v_{k}\right)$ where two consecutive vertices are related by a simple move. A path is closed if $v_{1}=v_{k}$. We distinguish three types of closed paths:

If three vertices (cut-systems) have $g-1$ curves $\gamma_{1}, \ldots, \gamma_{g-1}$ in common and if the remaining three curves $\gamma_{g}, \gamma_{g}^{\prime}, \gamma_{g}^{\prime \prime}$ intersect each other once, as on Figure 2, C3, then the vertices form a closed triangular path:

(C3) a triangle $\left\langle\gamma_{g}\right\rangle \rightarrow\left\langle\gamma_{g}^{\prime}\right\rangle \rightarrow\left\langle\gamma_{g}^{\prime \prime}\right\rangle \rightarrow\left\langle\gamma_{g}\right\rangle$.

If four vertices have $g-2$ curves $\gamma_{1}, \ldots, \gamma_{g-2}$ in common and the remaining pairs of curves consist of $\left(\gamma_{g-1}, \gamma_{g}\right),\left(\gamma_{g-1}^{\prime}, \gamma_{g}\right),\left(\gamma_{g-1}^{\prime}, \gamma_{g}^{\prime}\right),\left(\gamma_{g-1}, \gamma_{g}^{\prime}\right)$ where the curves intersect as on Figure 2, C4, then the vertices form a closed square path:

(C4) a square $\left\langle\gamma_{g-1}, \gamma_{g}\right\rangle \rightarrow\left\langle\gamma_{g-1}^{\prime}, \gamma_{g}\right\rangle \rightarrow\left\langle\gamma_{g-1}^{\prime}, \gamma_{g}^{\prime}\right\rangle \rightarrow\left\langle\gamma_{g-1}, \gamma_{g}^{\prime}\right\rangle \rightarrow\left\langle\gamma_{g-1}, \gamma_{g}\right\rangle$.

If five vertices have $g-2$ curves $\gamma_{1}, \ldots, \gamma_{g-2}$ in common and the remaining pairs of curves consist of $\left(\gamma_{g-1}, \gamma_{g}\right),\left(\gamma_{g-1}, \gamma_{g}^{\prime}\right),\left(\gamma_{g-1}^{\prime}, \gamma_{g}^{\prime}\right),\left(\gamma_{g-1}^{\prime}, \gamma_{g}^{\prime \prime}\right)$ and $\left(\gamma_{g}, \gamma_{g}^{\prime \prime}\right)$ where the curves intersect as on Figure 2, C5, then the vertices form a closed pentagon path:

Geometry and Topology, Volume 3 (1999) 
(C5) a pentagon $\left\langle\gamma_{g-1}, \gamma_{g}\right\rangle \rightarrow\left\langle\gamma_{g-1}, \gamma_{g}^{\prime}\right\rangle \rightarrow\left\langle\gamma_{g-1}^{\prime}, \gamma_{g}^{\prime}\right\rangle \rightarrow\left\langle\gamma_{g-1}^{\prime}, \gamma_{g}^{\prime \prime}\right\rangle \rightarrow$ $\left\langle\gamma_{g}^{\prime \prime}, \gamma_{g}\right\rangle \rightarrow\left\langle\gamma_{g-1}, \gamma_{g}\right\rangle$.

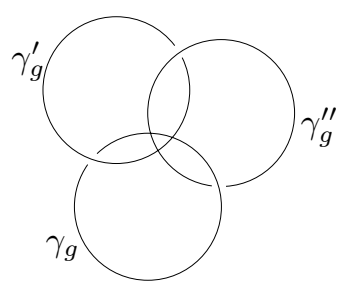

(C3)
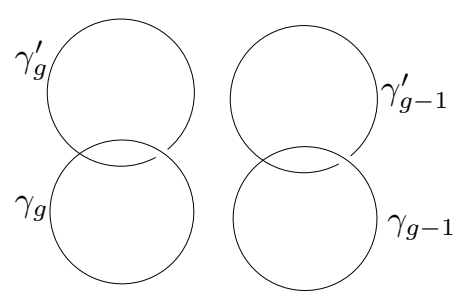

$(\mathrm{C} 4)$

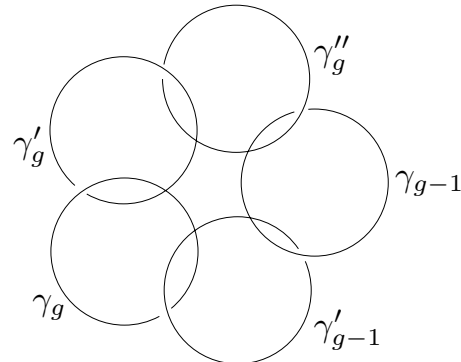

(C5)

Figure 2: Configurations of curves in paths (C3), (C4) and (C5)

$X$ is a 2 -dimensional cell complex obtained from $X^{1}$ by attaching a $2-$ cell to every closed edge-path of type (C3), (C4) or (C5). The mapping class group of $S$ acts on $S$ by homeomorphisms so it takes cut systems to cut systems. Since the edges and the faces of $X$ are determined by the intersections of pairs of curves, which are clearly preserved by homeomorphisms, the action on $X^{0}$ extends to a cellular action on $X$.

In this section we shall prove the main result of [9]:

Theorem $4 \quad X$ is connected and simply connected.

We want to prove that every closed path $\mathbf{p}$ is null-homotopic. If $\mathbf{p}$ is nullhomotopic we shall write $\mathbf{p} \sim \mathbf{o}$. We start with a closed path $\mathbf{p}=\left(v_{1}, \ldots, v_{k}\right)$ and try to simplify it. If $\mathbf{q}$ is a short-cut, an edge path connecting a vertex $v_{i}$ of $\mathbf{p}$ with $v_{j}, j>i$, we can split $\mathbf{p}$ into two closed edge-paths: $\mathbf{p}_{1}=\left(v_{j}, v_{j+1}, \ldots, v_{k}, v_{2}, \ldots, v_{i-1}, \mathbf{q}\right)$ and $\mathbf{p}_{2}=\left(\mathbf{q}^{-1}, v_{i+1}, v_{i+2}, \ldots, v_{j}\right)$.

If both paths are null-homotopic in $X$ then $\mathbf{p} \sim \mathbf{o}$. We want to prove Theorem 4 by splitting path $\mathbf{p}$ into simpler paths according to a notion of complexity which is described in the next definition.

Definition 2 Let $\mathbf{p}=\left(v_{1}, v_{2}, \ldots, v_{k}\right)$ be a path in $X$. Let $\alpha$ be a fixed curve of some fixed $v_{j}$. We define distance from $\alpha$ to a vertex $v_{i}$ to be $d\left(\alpha, v_{i}\right)=$ $\min \left\{|\alpha \cap \beta|: \beta \in v_{i}\right\}$. The radius of $\mathbf{p}$ around $\alpha$ is equal to the maximum distance from $\alpha$ to the vertices of $\mathbf{p}$. The path $\mathbf{p}$ is called a segment if every vertex of $\mathbf{p}$ contains a fixed curve $\alpha$. We shall write $\alpha$-segment if we want to stress the fact that $\alpha$ is the common curve of the segment. If the segment has several fixed curves we can write $(\alpha, \beta, \ldots, \gamma)$-segment. 
Theorem 4 will be proven by induction on the genus of $S$, for the given genus it will be proven by induction on the radius of a path $\mathbf{p}$ and for a given radius $m$ around a curve $\alpha$ we shall induct on the number of segments of $\mathbf{p}$ which have a common curve $\gamma$ with $|\gamma \cap \alpha|=m$. The main tool in the proof of Theorem 4 is a reduction of the number of intersection points of curves.

Definition 3 Curves $\alpha$ and $\beta$ on $S$ have an excess intersection if there exist curves $\alpha^{\prime}$ and $\beta^{\prime}$, isotopic to $\alpha$ and $\beta$ respectively, and such that $|\alpha \cap \beta|>$ $\left|\alpha^{\prime} \cap \beta^{\prime}\right|$. Curves $\alpha$ and $\beta$ form a 2-gon if there are arcs $a$ of $\alpha$ and $b$ of $\beta$, which meet only at their common end points and do not meet other points of $\alpha$ or $\beta$ and such that $a \cup b$ bound a disk, possibly with holes, on $S$. The disk is called a $2-$ gon. We can cut off the $2-$ gon from $\alpha$ by replacing the $\operatorname{arc} a$ of $\alpha$ by the arc $b$ of $\beta$. We get a new curve $\alpha^{\prime}$ (see Figure 3).

Lemma 5 (Hass-Scott, see [8], Lemma 3.1) If $\alpha, \beta$ are two curves on a surface $S$ having an excess intersection then they form a $2-$ gon (without holes) on $S$.

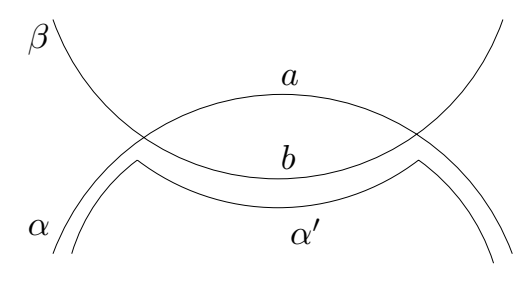

Figure 3: Curves $\alpha$ and $\beta$ form a 2-gon.

Corollary 5.1 Two simple closed geodesics on $S$ have no excess intersection. In particular if we replace two curves by geodesics in their isotopy class then the number of intersection points between the curves does not increase.

Proof If there is a 2-gon we can shorten one geodesic in its homotopy class, by first cutting off the 2 -gon and then smoothing corners.

Lemma 6 Consider a finite collection of simple closed geodesics on $S$. Suppose that curves $\alpha$ and $\beta$ of the collection form a minimal 2-gon (which does not contain another 2-gon). Let $\alpha^{\prime}$ be the curve obtained by cutting off the minimal 2-gon from $\alpha$ and passing to the isotopic geodesic. Then $\left|\alpha \cap \alpha^{\prime}\right|=0$, $\left|\beta \cap \alpha^{\prime}\right|<|\beta \cap \alpha|$ and $\left|\gamma \cap \alpha^{\prime}\right| \leq|\gamma \cap \alpha|$ for any other curve $\gamma$ of the collection. In particular if $|\gamma \cap \alpha|=1$ then $\left|\gamma \cap \alpha^{\prime}\right|=1$. Also $[\alpha]=\left[\alpha^{\prime}\right]$. 
Proof Since the 2-gon formed by arcs $a$ and $b$ of $\alpha$ and $\beta$ is minimal every other curve $\gamma$ of the collection intersects the 2-gon along arcs which meet $a$ and $b$ once. Thus cutting off the 2-gon will not change $|\alpha \cap \gamma|$ and it may only decrease after passing to the isotopic geodesic. Clearly $\alpha$ and $\alpha^{\prime}$ are disjoint and homologous on $\bar{S}$ and by passing to $\alpha^{\prime}$ we remove at least two intersection points of $\alpha$ with $\beta$.

\section{$2.1 \quad$ The case of genus 1}

In this section we shall assume that $S$ is a surface of genus one, possibly with boundary. By $\bar{S}$ we shall denote the closed torus obtained by glueing a disk to each boundary component of $S$. We want to prove:

Proposition 7 If $S$ has genus one then the cut system complex $X$ of $S$ is connected and simply connected.

On a closed torus $\bar{S}$ the homology class $[\alpha]$ of a curve $\alpha$ is defined by a pair of relatively prime integers, up to a sign, after a fixed choice of a basis of $H_{1}(\bar{S}, \mathbb{Z})$. If $[\alpha]=\left(a_{1}, a_{2}\right)$ and $[\beta]=\left(b_{1}, b_{2}\right)$ then the absolute value of their algebraic intersection number is equal $i(\alpha, \beta)=\left|a_{1} b_{2}-a_{2} b_{1}\right|$. If $\alpha$ and $\beta$ are geodesics on $\bar{S}$ then $|\alpha \cap \beta|=i(\alpha, \beta)$, therefore it is also true for curves on $S$ which form no 2 -gons, because then they have no excess intersection on $\bar{S}$.

Lemma 8 Let $\alpha, \beta$ be nonseparating curves on $S$ and suppose that $|\alpha \cap \beta|=$ $k \neq 1$. Then there exists a nonseparating curve $\delta$ such that if $k=0$ then $|\delta \cap \alpha|=|\delta \cap \beta|=1$ and if $k>1$ then $|\delta \cap \alpha|<k$ and $|\delta \cap \beta|<k$.

Proof If $|\alpha \cap \beta|=0$ then the curves are isotopic on $\bar{S}$ and they split $S$ into two connected components $S_{1}$ and $S_{2}$. We can choose points $P$ and $Q$ on $\alpha$ and $\beta$ respectively and connect them by simple arcs in $S_{1}$ and in $S_{2}$. The union of the arcs forms the required curve $\delta$. If $k>1$ and if the curves have an excess intersection on $\bar{S}$ then they form a 2 -gon on $S$. We can cut off the 2 -gon decreasing the intersection, by Lemma 6 . If there are no 2 -gons then the algebraic and the geometric intersection numbers are equal. In particular all intersections have the same sign. Consider two intersection points consecutive along $\beta$. Choose $\delta$ as on Figure 4. Then $|\delta \cap \alpha|=1$ so it is nonseparating and $|\delta \cap \beta|<k$. 


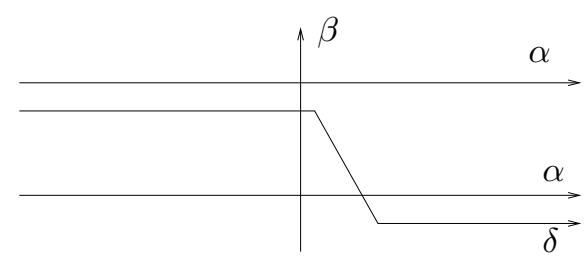

Figure 4: Curve $\delta$ has smaller intersection with $\alpha$ and $\beta$.

Corollary 8.1 If surface $S$ has genus 1 then the cut system complex of $S$ is connected.

Proof A cut system on $S$ is an isotopy class of a single curve. If two curves intersect once they are connected by an edge. It follows from the last lemma by induction that any two curves can be connected by an edge-path in $X$.

We now pass to a proof that closed paths are null-homotopic.

Lemma 9 A closed path $\mathbf{p}=\left(\delta_{1}, \delta_{2}, \delta_{3}, \delta_{4}, \delta_{1}\right)$ such that $\left|\delta_{2} \cap \delta_{4}\right|=0$ is nullhomotopic in $X$.

Proof Let $\beta=T_{\delta_{2}}^{ \pm 1}\left(\delta_{3}\right)$ be the image of $\delta_{3}$ by the Dehn Twist along $\delta_{2}$. Recall that as a set $\beta=\delta_{2} \cup \delta_{3}$ outside a small neighbourhood of $\delta_{2} \cap \delta_{3}$. From this we get that $\left|\beta \cap \delta_{2}\right|=1,\left|\beta \cap \delta_{3}\right|=1$ and $\left|\beta \cap \delta_{4}\right|=1$. Thus $\mathbf{p}^{\prime}=\left(\delta_{1}, \delta_{2}, \beta, \delta_{4}, \delta_{1}\right)$ is a closed path which is homotopic to $\mathbf{p}$ because $\mathbf{p}-\mathbf{p}^{\prime}$ splits into a sum of two triangles $\mathbf{t}_{\mathbf{1}}=\left(\beta, \delta_{2}, \delta_{3}, \beta\right)$ and $\mathbf{t}_{\mathbf{2}}=\left(\beta, \delta_{3}, \delta_{4}, \beta\right)$. We also have $i\left(\beta, \delta_{1}\right)=\left|i\left(\delta_{3}, \delta_{1}\right) \pm i\left(\delta_{2}, \delta_{1}\right)\right|$, so for a suitable choice of the sign of the twist we have $i\left(\delta_{3}, \delta_{1}\right)>i\left(\beta, \delta_{1}\right)$ unless $i\left(\delta_{3}, \delta_{1}\right)=0$. We may assume by induction that $i\left(\delta_{3}, \delta_{1}\right)=0$. If $\left|\delta_{1} \cap \delta_{3}\right|>0$ then $\delta_{1}$ and $\delta_{3}$ have an excess intersection on $\bar{S}$ and form a 2-gon on $S$. We can cut off the 2-gon from $\delta_{3}$ getting a new curve $\beta$ such that $[\beta]=\left[\delta_{3}\right],\left|\beta \cap \delta_{3}\right|=0,\left|\beta \cap \delta_{1}\right|<\left|\delta_{3} \cap \delta_{1}\right|$ and $\left|\beta \cap \delta_{i}\right|=\left|\delta_{3} \cap \delta_{i}\right|$ for $i \neq 1$, by Lemma 6 . We get a new closed path $\mathbf{p}^{\prime}=\left(\delta_{1}, \delta_{2}, \beta, \delta_{4}, \delta_{1}\right)$ and the difference between it and the old path is a closed path $\mathbf{q}=\left(\beta, \delta_{2}, \delta_{3}, \delta_{4}, \beta\right)$ with $\left|\beta \cap \delta_{3}\right|=0$ and $\left|\delta_{2} \cap \delta_{4}\right|=0$. So by induction it suffices to assume that $\left|\delta_{1} \cap \delta_{3}\right|=0$. If we now let $\beta=T_{\delta_{2}}\left(\delta_{3}\right)$ then $\beta$ intersects each of the four curves once so our path is a sum of four triangles and thus is null-homotopic in $X$.

Lemma 10 If $\mathbf{p}=\left(\alpha_{1}, \ldots, \alpha_{k}\right)$ is a closed path then there exists a closed path $\mathbf{p}^{\prime}=\left(\alpha_{1}^{\prime}, \ldots, \alpha_{k}^{\prime}\right)$ such that $\mathbf{p}^{\prime}$ is homotopic to $\mathbf{p}$ in $X,\left[\alpha_{i}\right]=\left[\alpha_{i}^{\prime}\right]$ for all $i$ and the collection of curves $\alpha_{1}^{\prime}, \ldots, \alpha_{k-1}^{\prime}$ forms no 2-gons. 
Proof Suppose that there exists a 2-gon bounded by arcs of two curves in $\mathbf{p}$. Then there also exists a minimal 2-gon bounded by arcs of curves $\alpha_{i}$ and $\alpha_{j}$. If we cut off the $2-$ gon from $\alpha_{i}$ we get a curve $\alpha_{i}^{\prime}$ such that $\left[\alpha_{i}\right]=\left[\alpha_{i}^{\prime}\right], \alpha_{i}^{\prime}$ is disjoint from $\alpha_{i},\left|\alpha_{j} \cap \alpha_{i}\right|>\left|\alpha_{j} \cap \alpha_{i}^{\prime}\right|$ and $\left|\alpha_{m} \cap \alpha_{i}^{\prime}\right| \leq\left|\alpha_{m} \cap \alpha_{i}\right|$ for any other curve $\alpha_{m}$, by Lemma 6 . It follows that if we replace $\alpha_{i}$ by $\alpha_{i}^{\prime}$ we get a new closed path $\mathbf{p}^{\prime}$ with a smaller number of intersection points between its curves. The difference of the two paths is a closed path $\mathbf{q}=\left(\alpha_{i-1}, \alpha_{i}, \alpha_{i+1}, \alpha_{i}^{\prime}, \alpha_{i-1}\right)$ which is null-homotopic by Lemma 9 . Thus $\mathbf{p}^{\prime}$ is homotopic to $\mathbf{p}$ in $X$. Lemma 10 follows by induction on the total number of intersection points between pairs of curves of $\mathbf{p}$.

Proof of Proposition 7 Let $\mathbf{p}=\left(\alpha_{1}, \ldots, \alpha_{k}, \alpha_{1}\right)$ be a closed path in $X^{1}$. We may assume that the path has no 2 -gons. By Lemma 5 there is no excess intersection on the closed torus $\bar{S}$. It means that the geometric intersection number of two curves of $\mathbf{p}$ is equal to their algebraic intersection number. Let $m=\max \left\{i\left(\alpha_{1}, \alpha_{j}\right) \mid j=1, \ldots, k\right\}$ be the radius of $\mathbf{p}$ around $\alpha_{1}$. Suppose first that $m=1$. Two disjoint curves on $\bar{S}$ have the same homology class, and two curves representing the same class have algebraic intersection equal to 0 . It follows that two consecutive curves in a path cannot be both disjoint from $\alpha_{1}$. If $k>4$ then either $\left|\alpha_{1} \cap \alpha_{3}\right|=1$ or $\left|\alpha_{1} \cap \alpha_{4}\right|=1$. We get an edge which splits $\mathbf{p}$ into two shorter closed paths with radius 1 . If $k=4$ and $\left|\alpha_{1} \cap \alpha_{3}\right|=0$ then $\mathbf{p} \sim \mathbf{o}$ by Lemma 9 . If $k=3$ then $\mathbf{p}$ is a triangle. Suppose now that $m>1$. We may assume by induction that every path of radius less than $m$ is null-homotopic and every path of radius $m$ which has less curves $\alpha_{j}$ with $\left|\alpha_{1} \cap \alpha_{j}\right|=m$ is also null-homotopic. Choose the smallest $i$ such that $i\left(\alpha_{1}, \alpha_{i}\right)=m$. Then $i\left(\alpha_{1}, \alpha_{i-1}\right)<m$ and $i\left(\alpha_{1}, \alpha_{i+1}\right) \leq m$. Choose a basis of the homology group $H_{1}(\bar{S})$ which contains the curve $\alpha_{1}$. A homology class of a curve is then represented by a pair of integers $(a, b)$. We consider the homology classes and their intersection numbers up to a sign. We have $\left[\alpha_{1}\right]=(1,0)$, $\left[\alpha_{i-1}\right]=(a, b),\left[\alpha_{i}\right]=(p, m)$ and $\left[\alpha_{i+1}\right]=(c, d)$. The intersection form is defined by $i((a, b),(c, d))=|a d-b c|$. Thus $a m-b p= \pm 1, c m-d p= \pm 1,|b|<$ $m,|d| \leq m$. We get $m(a d-b c)=( \pm d \pm b)$. Since $2 m>|b|+|d|$ we must have $|a d-b c|=1$ or $a d-b c=0$. In the first case $i\left(\alpha_{i-1}, \alpha_{i+1}\right)=\left|\alpha_{i-1} \cap \alpha_{i+1}\right|=1$. We can "cut off" the triangle $\mathbf{q}=\left(\alpha_{i-1}, \alpha_{i}, \alpha_{i+1}, \alpha_{i-1}\right)$ getting a path which is null-homotopic by the induction hypothesis. If $a d-b c=0$ then $i\left(\alpha_{i-1}, \alpha_{i+1}\right)=$ $\left|\alpha_{i-1} \cap \alpha_{i+1}\right|=0$. Let $\beta=T_{\alpha_{i-1}}^{ \pm 1}\left(\alpha_{i}\right)$. Then $\left|\beta \cap \alpha_{i-1}\right|=1$ and $\left|\beta \cap \alpha_{i+1}\right|=1$. We can replace $\alpha_{i}$ by $\beta$ getting a new closed path. Their difference is the closed path $\mathbf{q}=\left(\alpha_{i-1}, \alpha_{i}, \alpha_{i+1}, \beta, \alpha_{i-1}\right)$ which is null homotopic by lemma 9 . Thus the new path is homotopic to the old path. For a suitable choice of the sign of the Dehn twist we have $i\left(\beta, \alpha_{1}\right)<m$. It may happen that $\left|\beta \cap \alpha_{1}\right| \geq m$. We 
can get rid of 2-gons by Lemma 10 and thus get rid of the excess intersection. We get a homotopic path which is null-homotopic by the induction hypothesis. This concludes the proof of Proposition 7

\subsection{Paths of radius 0}

From now until the end of section 2, we assume that $S$ is a surface of genus $g>1$ with a finite number of boundary components. We denote by $X$ the cut-system complex of $S$. We assume:

Induction Hypothesis 1 The cut-system complex of a surface of genus less than $g$ is connected and simply-connected.

We want to prove that every closed path in $X$ is null-homotopic. We shall start with paths of radius zero. The simplest paths of radius zero are closed segments.

Lemma 11 A closed segment is null-homotopic in $X$.

Proof When we cut $S$ open along the common curve $\alpha$ the remaining curves of each vertex form a vertex of a closed path in the cut-system complex of a surface of a smaller genus. By Induction Hypothesis 1 it is a sum of paths of type (C3), (C4) and (C5) there. When we adjoin $\alpha$ to every vertex we get a splitting of the original paths into null-homotopic paths.

In a similar way we prove:

Lemma 12 If two vertices of $X$ have one or more curves in common we can connect them by a path all of whose vertices contain the common curves.

Proof If we cut $S$ open along the common curves the remaining collection of curves form two vertices of the cut-system complex on the new surface of smaller genus. They can be connected by a path. If we adjoin all the common curves to each vertex of this path we get a path in $X$ with the required properties.

Remark 2 If $\alpha$ and $\beta$ are two disjoint non-separating curves on $S$ then $\alpha \cup \beta$ does not separate $S$ if and only if $[\alpha] \neq[\beta]$. In this case the pair $\alpha, \beta$ can be completed to a cut-system on $S$. 
We shall now construct two simple types of null-homotopic paths in $X$.

Lemma 13 Let $\alpha_{1}, \alpha_{2}, \alpha_{3}$ be disjoint curves such that the union of any two of them does not separate $S$ but the union of all three separates $S$. Then there exist disjoint curves $\beta_{1}, \beta_{2}, \beta_{3}$ and a closed path

$$
\begin{aligned}
\left\langle\alpha_{1}, \alpha_{2}\right\rangle \rightarrow\left\langle\alpha_{1}, \beta_{2}\right\rangle \rightarrow\left\langle\alpha_{1}, \alpha_{3}\right\rangle \rightarrow\left\langle\beta_{1}, \alpha_{3}\right\rangle & \rightarrow\left\langle\alpha_{2}, \alpha_{3}\right\rangle \\
& \rightarrow\left\langle\alpha_{2}, \beta_{3}\right\rangle \rightarrow\left\langle\alpha_{2}, \alpha_{1}\right\rangle,
\end{aligned}
$$

which is null-homotopic in $X$.

Proof Let $\gamma_{3}, \ldots, \gamma_{g}$ be a cut system on a surface $S-\left(\alpha_{1} \cup \alpha_{2} \cup \alpha_{3}\right)$ (not connected), ie, a collection of curves which does not separate the surface any further. Then $\left\langle\alpha_{1}, \alpha_{2}, \gamma_{3}, \ldots, \gamma_{g}\right\rangle$ is a cut system on $S$. Let $S_{1}$ and $S_{2}$ be the components of a surface obtained by cutting $S$ open along all $\alpha_{i}$ 's and $\gamma_{j}$ 's. An arc connecting different components of the boundary does not separate the surface so we can find (consecutively) disjoint arcs $b_{1}$ connecting $\alpha_{1}$ with $\alpha_{2}$, $b_{2}$ connecting $\alpha_{2}$ with $\alpha_{3}$ and $b_{3}$ connecting $\alpha_{3}$ with $\alpha_{1}$ in $S_{1}$, and similar $\operatorname{arcs} b_{1}^{\prime}, b_{2}^{\prime}$ and $b_{3}^{\prime}$ in $S_{2}$ with the corresponding ends coinciding in $S$. The pairs of corresponding arcs form the required curves $\beta_{1}, \beta_{2}$ and $\beta_{3}$ and we get a closed path $\mathbf{p}$ described in (C6). Moreover the curves $\beta_{2}$ and $\beta_{3}$ are disjoint and $\left[\beta_{2}\right] \neq\left[\beta_{3}\right]$. To prove that the path is null-homotopic in $X$ we choose a curve $\delta=T_{\alpha_{2}}\left(\beta_{1}\right)$. Then $\left|\delta \cap \alpha_{1}\right|=\left|\delta \cap \alpha_{2}\right|=\left|\delta \cap \beta_{1}\right|=\left|\delta \cap \beta_{2}\right|=1$ and $\left|\delta \cap \alpha_{3}\right|=\left|\delta \cap \beta_{3}\right|=0$. Figure 5 shows how $\mathbf{p}$ splits into a sum of triangles (C3), squares (C4) and pentagons (C5) and therefore is null-homotopic in $X$.

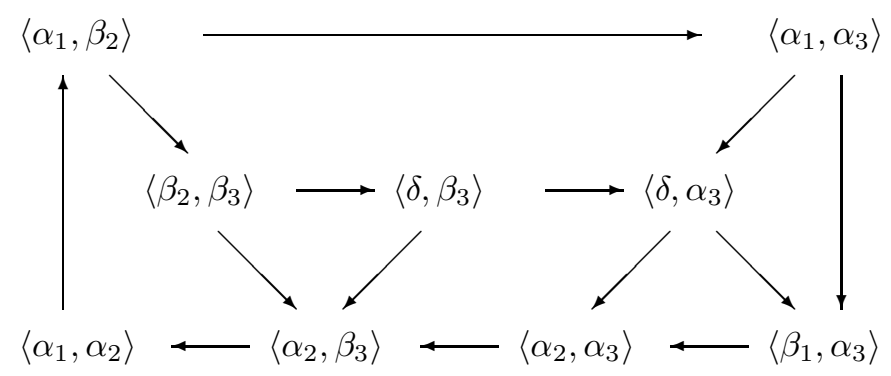

Figure 5: Hexagonal path

Proposition 14 If $\mathbf{p}$ is a path of radius 0 around a curve $\alpha$ then $\mathbf{p} \sim \mathbf{o}$. 
Proof Let $v_{0}$ be a vertex of $\mathbf{p}$ containing $\alpha$. We shall prove the proposition by induction on the number of segments of $\mathbf{p}$ having a fixed curve disjoint from $\alpha$. Consider the maximal $\alpha$-segment of $\mathbf{p}$ which contains the vertex $v_{0}$. We shall call it the first segment. Let $v_{1}$ be the last vertex of the first segment. The next vertex contains a curve $\beta$ disjoint from $\alpha$ such that $\beta$ is the common curve of the next segment of $\mathbf{p}$. Since $|\alpha \cap \beta|=0$ the simple move from $v_{1}$ to the next vertex does not involve $\beta$ hence $v_{1}$ also contains $\beta$. Let $v_{2}$ be the last vertex of the second segment. If there are only two segments then $v_{2}$ also contains both $\alpha$ and $\beta$. By Lemma 12 there is an $(\alpha, \beta)$-segment connecting $v_{1}$ and $v_{2}$. Then $\mathbf{p}$ is a sum of a closed $\alpha$-segment and a closed $\beta$-segment. So we may assume that there is a third segment. The vertex $\tilde{v}$ of $\mathbf{p}$ following $v_{2}$ contains a curve $\gamma$ disjoint from $\alpha$ and $\gamma$ is the common curve of the third segment. Let $v_{3}$ be the last vertex of the third segment. We shall reduce the number of segments. There are three cases.

Case 1 Vertex $v_{2}$ does not contain $\gamma$. Since $\tilde{v}$ contains $\gamma$ and does not contain $\beta$ we have $|\beta \cap \gamma|=1$. Let $S_{1}$ be a surface of genus $g-1$ obtained by cutting $S$ open along $\beta \cup \gamma$. Vertices $v_{2}$ and $\tilde{v}$ have $g-1$ curves in common and the common curves form a cut system $u$ on $S_{1}$. The union $\beta \cup \gamma$ cannot separate $S-\alpha$ hence $\alpha$ does not separate $S_{1}$ and it belongs to a cut-system $u^{\prime}$ on $S_{1}$. Vertices $u$ and $u^{\prime}$ can be connected by a path $\mathbf{q}$ in the cut-system complex of $S_{1}$. If we adjoin $\beta$ (respectively $\gamma$ ) to each vertex of $\mathbf{q}$ we get a path $\mathbf{q}_{2}$ (respectively $\mathbf{q}_{1}$ ). Path $\mathbf{q}_{2}$ connects $v_{2}$ to a vertex $u_{2}$ containing $\alpha$ and $\beta$ and path $\mathbf{q}_{1}$ connects $\tilde{v}$ to a vertex $u_{1}$ containing $\alpha$ and $\gamma$. The corresponding vertices of $\mathbf{q}_{1}$ and $\mathbf{q}_{2}$ are connected by an edge so the middle rectangle on Figure 6 splits into a sum of squares of type (C4) and is null-homotopic. We can connect $v_{1}$ to $u_{2}$ by an $(\alpha, \beta)$-segment so the triangle on Figure 6 is a closed $\beta$-segment and is also null-homotopic. The part of $\mathbf{p}$ between $v_{1}$ and $\tilde{v}$ can be replaced by the lower path on Figure 6 . We get a new path $\mathbf{p}^{\prime}$, which has a smaller number of segments (no $\beta$-segment) and is homotopic to $\mathbf{p}$ in $X$.

Case 2 Vertex $v_{2}$ contains $\gamma$ and $\alpha \cup \gamma$ does not separate $S$. If there exists a vertex $v$ which contains $\alpha$ and $\beta$ and $\gamma$ we can connect it to $v_{1}$ and $v_{2}$ using Lemma 12. We get a closed segment and the remaining path has one segment less (Figure 7). Otherwise $\alpha \cup \beta \cup \gamma$ separate $S$ and we can apply Lemma 13 . There exist vertices $w_{1}$ containing $\alpha$ and $\beta, w_{2}$ containing $\beta$ and $\gamma$ and $w_{3}$ containing $\alpha$ and $\gamma$ and a $\beta$-segment from $w_{1}$ to $w_{2}$, a $\gamma$-segment from $w_{2}$ to $w_{3}$ and an $\alpha$-segment from $w_{3}$ to $w_{1}$. The sum of the segments is nullhomotopic. We now connect $v_{1}$ to $w_{1}$ by an $(\alpha, \beta)$-segment and $v_{2}$ to $w_{2}$ by 


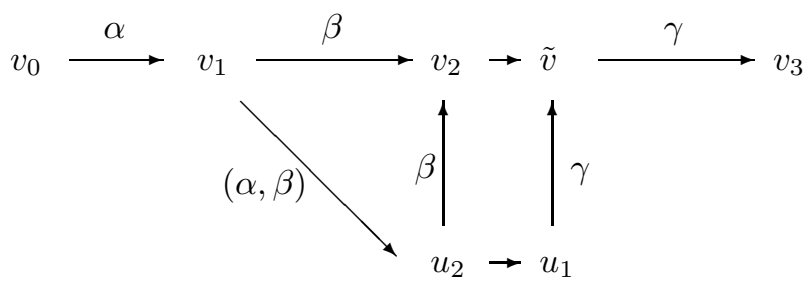

Figure 6: Path of radius 0, Case 1

a $(\beta, \gamma)$-segment. Thus the second segment of $\mathbf{p}$ can be replaced by a sum of an $\alpha$-segment and a $\gamma$-segment, and the difference is a closed $\beta$-segment plus a null-homotopic hexagonal path of Lemma 13 (see the right side of Figure 7).
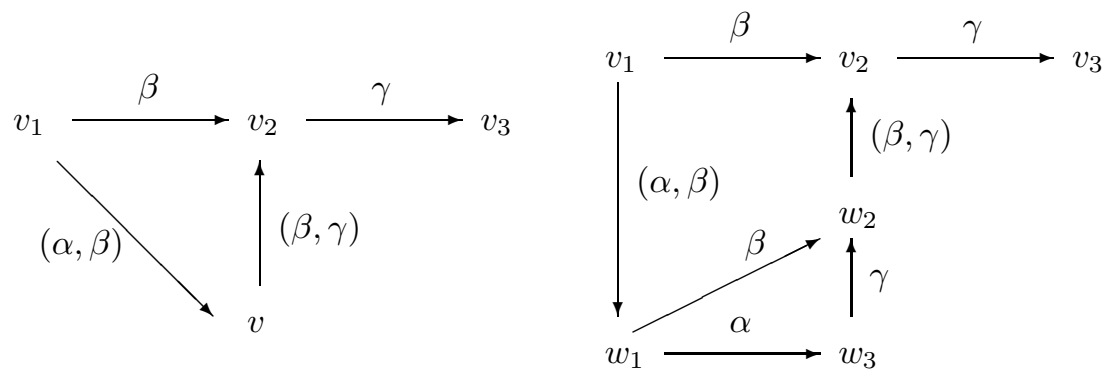

Figure 7: Path of radius 0, Case 2

Case 3 Vertex $v_{2}$ contains $\gamma$ and $\alpha \cup \gamma$ separates $S$ into two surfaces $S_{1}$ and $S_{2}$. If there were only three segments then, as at the vertex $v_{1}$, the first vertex of the first segment would contain both $\alpha$ and $\gamma$ and their union would not separate $S$. This contradicts our assumptions. It follows in particular that every closed path of radius zero with at most three segments (where the common curve of each segment is disjoint from a fixed curve of the first segment) is null-homotopic. We may assume that the path $\mathbf{p}$ has a fourth segment with a fixed curve $\delta$ disjoint from $\alpha$. Since $[\gamma]=[\alpha]$ we cannot have $|\gamma \cap \delta|=1$. Therefore $\delta$ is not involved in the simple move from $v_{3}$ to the next vertex and $v_{3}$ contains $\delta$. In particular $[\gamma] \neq[\delta]$ and $[\alpha] \neq[\delta]$. We may assume that $\beta$ lies in $S_{1}$. If $\delta$ lies in $S_{2}$ then there is a vertex $w$ which contains $\alpha$ and $\beta$ and $\delta$. We can connect $w$ to $v_{1}$ by an $(\alpha, \beta)$-segment, to $v_{2}$ by a $\beta$-segment and to $v_{3}$ by a $\delta$-segment. We get a new path, homotopic to $\mathbf{p}$, which does not contain $\beta$-segment nor $\gamma$-segment (see Figure 8, left part.) 
Suppose now that $\delta$ lies in $S_{1}$. Consider the cut-system complex $X_{1}$ of $S_{1}$ and choose a vertex $s$ of $X_{1}$ which contains $\delta$ and a vertex $s^{\prime}$ of $X_{1}$ which contains $\beta$. Let $\mathbf{q}$ be a path in $X_{1}$ which connects $s$ to $s^{\prime}$. Let $t$ be a fixed vertex of the cut-system complex $X_{2}$ of $S_{2}$ (if $X_{2}$ is not empty.) We add $\alpha$ and all curves of $t$ to each vertex of the path $\mathbf{q}$ and get an $\alpha$-segment in $X$ connecting a vertex $w_{2}$, containing $\delta$, to a vertex $w_{2}^{\prime}$, containing $\beta$. Then we add $\gamma$ and all curves of $t$ to each vertex of the path $\mathbf{q}$ and get a $\gamma$-segment in $X$ connecting a vertex $w_{3}$, containing $\delta$, to a vertex $w_{3}^{\prime}$, containing $\beta$. We now connect $v_{1}$ to $w_{2}$ by an $\alpha$-segment, $v_{2}$ to $w_{2}^{\prime}$ by a $\beta$-segment, $v_{3}$ to $w_{3}^{\prime}$ by a $\gamma$-segment and $v_{4}$ to $w_{3}$ by a $\delta$-segment (see Figure 8 , the right side.) Corresponding vertices of the two vertical segments on Figure 8, the right side, have a common curve $\delta_{i}$, a curve of a vertex of the path $\mathbf{q}$ disjoint from $\alpha$ and from $\gamma$, and can be connected by a $\delta_{i}$-segment. We get a "ladder" such that each small rectangle in this ladder has radius zero around $\gamma$ and consists of only three segments. Therefore it is null-homotopic. Every other closed path on Figure 8, the right side, has a similar property. We get a new path, homotopic to $\mathbf{p}$, which does not contain $\beta$-segment nor $\gamma$-segment.

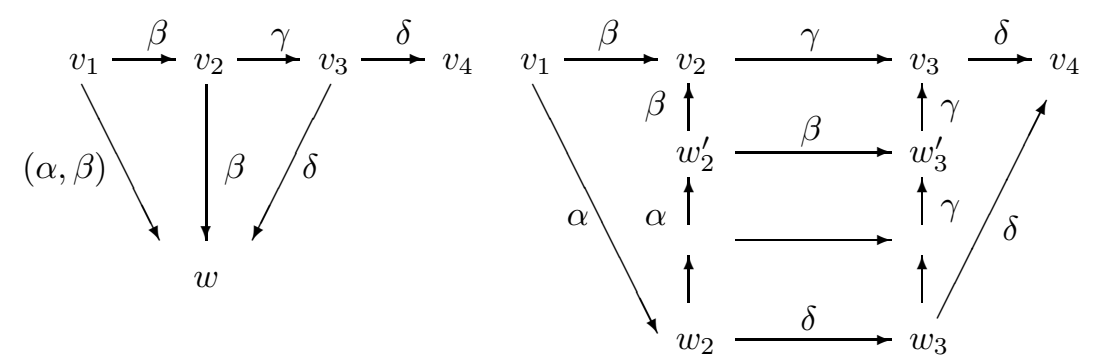

Figure 8: Path of radius 0, Case 3

\subsection{The general case}

We now pass to the general case and we want to prove it by induction on the radius of a closed path.

Induction hypothesis 2 A closed path of radius less than $m$ is null-homotopic.

We want to prove that a closed path $\mathbf{p}$ of radius $m$ around a curve $\alpha$ is nullhomotopic. The general idea is to construct a short-cut, an edge-path which 
splits $\mathbf{p}$ and is close to $\alpha$ and to a fixed $\beta$-segment. The first step is to construct one intermediate curve.

Lemma 15 Let $\gamma_{1}$ and $\gamma_{2}$ be non-separating curves on $S$ such that $\left|\gamma_{1} \cap \gamma_{2}\right|=$ $n>1$. Then there exists a non-separating curve $\delta$ such that $\left|\gamma_{1} \cap \delta\right|<n$ and $\left|\gamma_{2} \cap \delta\right|<n$. Suppose that we are also given non-separating curves $\alpha, \beta$ and an integer $m>0$ such that $|\alpha \cap \beta| \leq m,\left|\gamma_{1} \cap \alpha\right|<m,\left|\gamma_{2} \cap \alpha\right| \leq m$, and $\left|\beta \cap \gamma_{1}\right|=\left|\beta \cap \gamma_{2}\right|=0$. Then we can find a curve $\delta$ as above which also satisfies $|\delta \cap \alpha|<m$ and $|\delta \cap \beta|=0$.

Proof We orient the curves $\gamma_{1}$ and $\gamma_{2}$ and split the union $\gamma_{1} \cup \gamma_{2}$ into a different union of oriented simple closed curves as follows. We start near an intersection point, say $P_{1}$, on the side of $\gamma_{2}$ after $\gamma_{1}$ crosses it and on the side of $\gamma_{1}$ before $\gamma_{2}$ crosses it. Now we move parallel to $\gamma_{1}$ to the next intersection point with $\gamma_{2}$, say $P_{2}$. We do not cross $\gamma_{2}$ at $P_{2}$ and move parallel to $\gamma_{2}$, in the positive direction, back to $P_{1}$. We get a curve $\delta_{1}$. Now we start near $P_{2}$ and move parallel to $\gamma_{1}$ until we meet an intersection point, say $P_{3}$, which is either equal to $P_{1}$ or was not met before. We do not cross $\gamma_{2}$ at $P_{3}$ and move parallel to $\gamma_{2}$, in the positive direction, back to $P_{2}$. We get a curve $\delta_{2}$. And so on. Curve $\delta_{i}$ meets $\gamma_{1}$ near some points of $\gamma_{1} \cap \gamma_{2}$, but not near $P_{i}$ and it meets $\gamma_{2}$ near some points of $\gamma_{1} \cap \gamma_{2}$, but not near $P_{i+1}$. So $\delta_{i}$ meets both curves less than $n$ times. Let $\bar{\gamma}$ denote the (oriented) homology class represented by an oriented curve $\gamma$ in $H_{1}(\bar{S}, \mathbb{Z})$. We have $\bar{\gamma}_{1}+\bar{\gamma}_{2}=\bar{\delta}_{1}+\ldots+\bar{\delta}_{k}$. Now we repeat a similar construction for the opposite orientation of $\gamma_{2}$ starting near the same point $P_{1}$. We get new curves $\epsilon_{1}, \ldots, \epsilon_{r}$ and $\bar{\gamma}_{1}-\bar{\gamma}_{2}=\bar{\epsilon}_{1}+\ldots+\bar{\epsilon}_{r}$. Also $\bar{\delta}_{1}-\bar{\epsilon}_{1}=\bar{\gamma}_{2}$. Combining these equalities in $H_{1}(\bar{S}, \mathbb{Z})$ we get $\bar{\epsilon}_{1}+\sum_{i \neq 1} \bar{\delta}_{i}=\bar{\gamma}_{1}$, $\bar{\delta}_{1}+\sum_{i \neq 1} \bar{\epsilon}_{i}=\bar{\gamma}_{1}, \sum_{i \neq 1} \bar{\delta}_{i}-\sum_{i \neq 1} \bar{\epsilon}_{i}=\bar{\gamma}_{2}$. A simple closed curve separates $S$ if and only if it represents 0 in $H_{1}(\bar{S}, \mathbb{Z})$. Since $\gamma_{1}$ and $\gamma_{2}$ are non-separating it follows that either $\delta_{1}$ and some $\delta_{i}, i \neq 1$, are not separating or $\epsilon_{1}$ and some $\epsilon_{i}, i \neq 1$, are not separating. And each of them meets $\gamma_{1}$ and $\gamma_{2}$ less than $n$ times, so it can be chosen for $\delta$. If we are also given curves $\alpha$ and $\beta$ and integer $m>0$ which satisfy the assumptions of the Lemma then $\left|\gamma_{1} \cap \alpha\right|+\left|\gamma_{2} \cap \alpha\right|=$ $\Sigma\left|\delta_{i} \cap \alpha\right|=\Sigma\left|\epsilon_{i} \cap \alpha\right| \leq 2 m-1$ therefore one of the constructed nonseparating curves intersects $\alpha$ less than $m$ times and is disjoint from $\beta$.

Lemma 16 Let $\gamma_{1}$ and $\gamma_{2}$ be disjoint non-separating curves on $S$ such that $\gamma_{1} \cup \gamma_{2}$ separates $S$. Then there exists a non-separating curve $\delta$ such that $\left|\gamma_{1} \cap \delta\right|=1$ and $\left|\gamma_{2} \cap \delta\right|=1$. Suppose that we are also given non-separating curves $\alpha, \beta$ and an integer $m>0$ such that $|\alpha \cap \beta| \leq m,|\alpha \cap \beta|=1$ if $m=1$, 
$\left|\gamma_{1} \cap \alpha\right|<m,\left|\gamma_{2} \cap \alpha\right| \leq m$, and $\left|\beta \cap \gamma_{1}\right|=\left|\beta \cap \gamma_{2}\right|=0$. Then we can find a curve $\delta$ as above which also satisfies $|\delta \cap \alpha|<m$ and $|\delta \cap \beta|<m$.

Proof By our assumptions $\gamma_{1} \cup \gamma_{2}$ separates $S$ into two components $S_{1}$ and $S_{2}$. We can choose a simple arc $d_{1}$ in $S_{1}$ which connects $\gamma_{1}$ to $\gamma_{2}$ and a simple arc $d_{2}$ in $S_{2}$ which connects $\gamma_{1}$ to $\gamma_{2}$. Then we can slide the end-points of $d_{1}$ and $d_{2}$ along $\gamma_{1}$ and $\gamma_{2}$ to make the end-points meet. We get a nonseparating curve $\delta$ which intersects $\gamma_{1}$ and $\gamma_{2}$ once. Suppose that we are also given curves $\alpha$ and $\beta$ and an integer $m>0$. We need to alter the $\operatorname{arcs} d_{1}$ and $d_{2}$, if necessary, in order to decrease the intersection of $\delta$ with $\alpha$ and $\beta$. We may assume that $\beta$ lies in $S_{1}$. We have to consider several cases.

Case 1 Curve $\alpha$ lies in $S_{1}$. Then $d_{2}$ is disjoint from $\alpha$. If $m=1$ then $|\alpha \cap \beta|=1$ so the union $\alpha \cup \beta$ does not separate its regular neighbourhood and does not separate $S_{1}$. We can choose $d_{1}$ disjoint from $\alpha$ and $\beta$ and then $\delta$ is also disjoint from $\alpha$ and $\beta$.

Suppose that $m>1$. If $\alpha$ separates $S_{1}$ (but does not separate $S$ ) then it separates $\gamma_{1}$ from $\gamma_{2}$ in $S_{1}$. There exists an arc $d$ in $S_{1}$ which connects $\gamma_{1}$ with $\gamma_{2}$ and is disjoint from $\alpha$, if $\alpha$ does not separate $S_{1}$, or meets $\alpha$ once, if $\alpha$ separates $S_{1}$. We choose such an arc $d$ which has minimal number of intersections with $\beta$. If $|\beta \cap d|>m$ then there exist two points $P$ and $Q$ of $\beta \cap d$, consecutive along $\beta$, and not separated by a point of $\beta \cap \alpha$. We can move along $d$ to $P$ then along $\beta$, without crossing $\beta$, to $Q$, and then continue along $d$ to its end. This produces an arc which meets $\alpha$ at most once and has smaller number of intersections with $\beta$. So we may assume that $|\beta \cap d|=m$ and that every pair of points of $\beta \cap d$ consecutive along $\beta$ is separated by a point of $\beta \cap \alpha$. We now alter $d$ as follows. Consider the intersection $d \cap(\alpha \cup \beta)$. If the first or the last point along $d$ of this intersection belongs to $\alpha$ we start from this end of $d$. Otherwise we start from any end. We move along $d$ to the first point, say $P$, of intersection with $\alpha \cup \beta$. If $P \in \alpha$ we continue along $\alpha$, without crossing it, to the next point of $\alpha \cap \beta$. Then along $\beta$, without crossing it, to the last point, say $Q$, of $\beta \cap d$ on $d$, and then along $d$ to its end. The new arc crosses $\beta$ at most once, near $Q$, and crosses $\alpha$ less than $m$ times. If $P \in \beta$ we continue along $\beta$, without crossing $\beta$, to $Q$ and then along $d$ to its end, which produces a similar result. We can choose such an arc for $d_{1}$ and then the curve $\delta$ satisfies the Lemma.

Case 2 Curve $\alpha$ meets $\gamma_{1}$ or $\gamma_{2}$. Then $m>1$, because if $m=1$ and $\left|\gamma_{1} \cap \alpha\right|=0$ and $\alpha$ crosses $\gamma_{2}$ into $S_{1}$ then it must cross it again in order to 
exit $S_{1}$, and this contradicts $\left|\gamma_{2} \cap \alpha\right| \leq m$. The arcs of $\alpha$ split $S_{1}$ (and $S_{2}$ ) into connected components. One of the components must meet both $\gamma_{1}$ and $\gamma_{2}$ (Otherwise the union of all components meeting $\gamma_{1}$ has $\alpha$ for a boundary component and then $\alpha$ is disjoint from $\gamma_{1}$ and $\gamma_{2}$.) Choosing $d_{1}$ (respectively $\left.d_{2}\right)$ in such a component we can make them disjoint from $\alpha$. Now we want to modify $d_{1}$ in such a way that $\left|d_{1} \cap \alpha\right|=0$ and $\left|d_{1} \cap \beta\right|<m$. There are three subcases.

Case 2a There exists an arc $a_{1}$ of $\alpha$ in $S_{1}$ which connects $\gamma_{1}$ and $\gamma_{2}$. Choose $d_{1}$ parallel to this arc. It may happen that $d_{1}$ meets $\beta m$ times. Then $a_{1}$ is the only arc of $\alpha$ which meets $\beta$. We then modify $d_{1}$ as follows. We move from $\gamma_{1}$ along $d_{1}$ until it meets $\beta$. Then we turn along $\beta$, away from $a_{1}$, to the next point of $a_{1}$. We turn before crossing $a_{1}$ and move parallel to $a_{1}$ to $\gamma_{2}$. The new arc does not meet $\alpha$ and meets $\beta$ less than $m$ times.

Case $2 \mathbf{b}$ There exists an arc of $\alpha$ in $S_{1}$ which connects $\gamma_{1}$ and $\beta$ and there exists an arc of $\alpha$ which connects $\gamma_{2}$ and $\beta$. Then there exist points $P$ and $Q$ of $\alpha \cap \beta$, consecutive along $\beta$, and $\operatorname{arcs} a_{1}$ and $a_{2}$ of $\alpha$ such that $a_{1}$ connects $\gamma_{1}$ to $P$ and $a_{2}$ connects $Q$ to $\gamma_{2}$. We move along $a_{1}$ to $P$ then along $\beta$, without crossing $\beta$, to $Q$, and then along $a_{2}$ to $\gamma_{2}$. The new arc does not meet $\alpha$ and meets $\beta$ less than $m$ times.

Case 2c If an arc of $\alpha$ in $S_{1}$ meets $\beta$ then it meets only $\gamma_{1}$. (The case of $\gamma_{2}$ is similar.) We consider an arc $d$ in $S_{1}$ which is disjoint from $\alpha$ and connects $\gamma_{1}$ and $\gamma_{2}$. We start at $\gamma_{2}$ and move along $d$ to the first point of intersection with $\beta$. Then we move along $\beta$, without crossing it, to the first point of intersection with $\alpha$. Then we move along $\alpha$, away from $\beta$, to $\gamma_{1}$. The new arc does not meet $\alpha$ and meets $\beta$ less than $m$ times. If $\beta$ is disjoint from $\alpha$ then $\beta$ is either disjoint from a component of $S_{1}-\alpha$ which connects $\gamma_{1}$ to $\gamma_{2}$ or is contained in it. We can find an arc in the component (disjoint from $\alpha$ ) which connects $\gamma_{1}$ with $\gamma_{2}$ and meets $\beta$ at most once.

So in each case we have an arc $d_{1}$ which is disjoint from $\alpha$ and meets $\beta$ less than $m$ times. We now slide the end-points of $d_{1}$ along $\gamma_{1}$ and $\gamma_{2}$ to meet the end-points of $d_{2}$. Each slide can be done along one of two arcs of $\gamma_{i}$. Choosing suitably we may assume that $d_{1}$ meets at most $m / 2$ points of $\alpha$ sliding along $\gamma_{2}$ and at most $(m-1) / 2$ points of $\alpha$ sliding along $\gamma_{1}$. The curve $\delta$ obtained from $d_{1}$ and $d_{2}$ meets $\alpha$ and $\beta$ less than $m$ times.

Case 3 The curve $\alpha$ lies in $S_{2}$. Then $|\alpha \cap \beta|=0$ so we must have $m>1$. We can choose $d_{2}$ which is disjoint from $\beta$ and meets $\alpha$ at most once and we 
can choose $d_{1}$ which is disjoint from $\alpha$ and meets $\beta$ at most once. The curve $\delta$ obtained from $d_{1}$ and $d_{2}$ meets $\alpha$ and $\beta$ less than $m$ times.

Lemma 17 Let $\delta_{1}$ and $\delta_{2}$ be non-separating curves on $S$ and let $w_{1}$ be a vertex of $X$ containing $\delta_{1}$ and let $w_{2}$ be a vertex of $X$ containing $\delta_{2}$. Then there exists an edge-path $\mathbf{q}=\left(w_{1}=z_{1}, z_{2}, \ldots, z_{k}=w_{2}\right)$ connecting $w_{1}$ and $w_{2}$. Suppose that we are also given non-separating curves $\alpha, \beta$ and an integer $m>0$ such that $|\alpha \cap \beta| \leq m,|\alpha \cap \beta|=1$ if $m=1,\left|\delta_{1} \cap \alpha\right|<m,\left|\delta_{2} \cap \alpha\right| \leq m$, and $\left|\beta \cap \delta_{1}\right|=\left|\beta \cap \delta_{2}\right|=0$. Then there exists a path $\mathbf{q}$ as above and an integer $j, 1 \leq j<k$, such that $d\left(z_{i}, \beta\right)<m$ for all $i, d\left(z_{i}, \alpha\right)<m$ for $1 \leq i \leq j<k$ and $z_{i}$ contains $\delta_{2}$ for $j<i \leq k$.

Proof We shall prove the lemma by induction on $\left|\delta_{1} \cap \delta_{2}\right|=n$.

If $\delta_{1}=\delta_{2}$ we can connect $w_{1}$ and $w_{2}$ by a $\delta_{1}$-segment, by Lemma 12 .

If $n=1$ there exist vertices $u_{1}, u_{2}$ in $X$ which are connected by an edge and such that $\delta_{1} \in u_{1}, \delta_{2} \in u_{2}$. Now we can connect $u_{1}$ to $w_{1}$ and $w_{2}$ to $u_{2}$ as in the previous case.

If $n=0$ and $\delta_{2} \cup \delta_{1}$ does not separate $S$ then there exists a vertex $v$ containing both curves $\delta_{2}$ and $\delta_{1}$. We can connect $v$ to $w_{1}$ and $w_{2}$ as in the first case.

Suppose now that $n=0$ and that $\delta_{2} \cup \delta_{1}$ separates $S$. Then, by Lemma 16 , there exists a curve $\delta$ such that $\left|\delta_{2} \cap \delta\right|=\left|\delta_{1} \cap \delta\right|=1$. We can find a vertex $v$ containing $\delta$ and we can connected $v$ to $w_{1}$ and $w_{2}$ as in the second case. If we are also given curves $\alpha$, and $\beta$ and an integer $m$ we can choose $\delta$ which also satisfies $|\alpha \cap \delta|<m$ and $|\beta \cap \delta|<m$. Then the path obtained by connecting $v$ to $w_{1}$ and $w_{2}$ have all vertices in a distance less than $m$ from $\beta$ and in a distance less than $m$ from $\alpha$, except for the final $\delta_{2}$-segment which ends at $w_{2}$ (Curve $\delta_{2}$ may have distance $m$ from $\alpha$.)

If $n>1$ then by Lemma 15 there exists a curve $\delta$ such that $\left|\delta_{1} \cap \delta\right|<n$ and $\left|\delta_{2} \cap \delta\right|<n$. We choose a vertex $v$ containing $\delta$. By induction on $n$ we can connect $v$ to $w_{1}$ and $w_{2}$. If we are also given curves $\alpha$ and $\beta$ and an integer $m$ then we can find $\delta$ which also satisfies $|\delta \cap \alpha|<m$ and $|\delta \cap \beta|=0$. By induction on $n$ we can connect $w_{1}$ to $v$ and $v$ to $w_{2}$ by a path the vertices of which are closer to $\beta$ than $m$, and closer to $\alpha$ than $m$ except for a final $\delta_{2}$-segment which ends at $w_{2}$.

As an immediate corollary we get:

Corollary 17.1 Complex $X$ is connected. 
We need one more lemma before we prove that every closed path is nullhomotopic in $X$.

Lemma 18 Let $\alpha, \beta, \gamma$ be non-separating curves on $S$ such that $|\alpha \cap \beta|=m$, $|\alpha \cap \gamma| \leq m,|\beta \cap \gamma|=1$. There exists a non-separating curve $\delta$ such that $|\delta \cap \alpha|<m,|\delta \cap \beta|=0$ and $|\delta \cap \gamma| \leq 1$. If $m=1$ then $|\delta \cap \gamma|=0$ and $[\delta]$ is different from $[\alpha],[\beta]$ and $[\gamma]$.

Proof When we split $S$ along $\gamma \cup \beta$ we get a surface $S_{1}$ with a "rectangular" boundary component $\partial$ consisting of two $\beta$-edges (vertical) and two $\gamma$-edges (horizontal on pictures of Figure 9). We can think of $S_{1}$ as a rectangle with holes and with some handles attached to it. Curve $\alpha$ intersects $S_{1}$ along some $\operatorname{arcs} a_{i}$ with end-points $P_{i}$ and $Q_{i}$ on $\partial$. If, for some $i$, points $P_{i}$ and $Q_{i}$ lie on the same $\beta$-edge then $m>1$ and we can construct a curve $\delta$ consisting of an arc parallel to $a_{i}$ and an arc parallel to the $\operatorname{arc}$ of $\beta$ which connects $P_{i}$ and $Q_{i}$ passing through the point $\gamma \cap \beta$. Then $|\delta \cap \beta|=0,|\delta \cap \alpha|<m$ and $|\delta \cap \gamma|=1$. Recall that if two curves intersect exactly at one point then they are both non-separating on $S$. Therefore $\delta$ satisfies the conditions of the Lemma. If for some $i$ points $P_{i}$ and $Q_{i}$ lie on different $\gamma$-edges of $\partial$ then we can modify the arc $a_{i}$ sliding its end-point $P_{i}$ along the $\gamma$ edge to the point corresponding to $Q_{i}$. We get a closed curve $\delta$ satisfying the conditions of the Lemma. So we may assume that there are no $\operatorname{arcs} a_{i}$ of the above types.

Suppose that for every pair $i, j$ the pairs of end-points $P_{i}, Q_{i}$ and $P_{j}, Q_{j}$ do not separate each other on $\partial$. Then we can connect the corresponding end points by nonintersecting intervals inside a rectangle. In the other words a regular neighbourhood of $\alpha \cup \partial$ in $S_{1}$ is a planar surface homeomorphic to a rectangle with holes. Since $S_{1}$ has positive genus there exists a subsurface of $S_{1}$ of a positive genus attached to one hole or a subsurface of $S_{1}$ which connects two holes of the rectangle. Such a subsurface contains a curve $\delta$ which is nonseparating on $S$ and is disjoint from $\alpha, \beta$ and $\gamma$ and the homology class $[\delta]$ is different from $[\alpha],[\beta]$ and $[\gamma]$. This happens in particular when $m=1$ because then there is at most one point on every edge and the pairs of end points of arcs do not separate each other.

So we may assume that $m>1$ and that there exists a pair of arcs, say $a_{1}$ and $a_{2}$, such that the pair $P_{1}, Q_{1}$ separates the pair $P_{2}, Q_{2}$ in $\partial$. Since an arc $a_{i}$ does not connect different $\gamma$-edges we must have two points, say $P_{1}$ and $P_{2}$, on the same edge. Suppose that they lie on a $\beta$-edge, say the left edge. Choosing an intermediate point, if there is one, we may assume that $P_{1}$ and $P_{2}$ are consecutive points of $\alpha$ along $\beta$. We have different possible configurations 
of pairs of points. For each of them we construct curves $\delta_{i}$, as on Figure 9. Each $\delta_{i}$ is disjoint from $\beta$ and intersects $\gamma$ at most once, and if it is disjoint from $\gamma$ it intersects some other curve once. So $\delta_{i}$ is not-separating. We shall prove that we can always choose a suitable $\delta_{i}$ with $\left|\delta_{i} \cap \alpha\right|<m$. Observe that $\delta_{i}$ may meet $\alpha$ only along the boundary $\partial$, and not along the arc connecting $P_{1}$ to $P_{2}$.

Case 1 Points $Q_{1}$ and $Q_{2}$ lie on the same $\gamma$-edge, say lower edge. If there is no point of $\alpha$ on $\gamma$ to the left of $Q_{1}$ then $\left|\delta_{1} \cap \alpha\right|<m$. If there is a point of $\alpha$ on $\gamma$ to the left of $Q_{1}$ then $\left|\delta_{2} \cap \alpha\right|<m$.

Case 2 Points $Q_{1}$ and $Q_{2}$ lie on different $\gamma$-edges. Then $\left|\delta_{3} \cap \alpha\right|<m$.

Case 3 Points $Q_{1}$ and $Q_{2}$ lie on the right edge. Then $\left|\delta_{4} \cap \alpha\right|<m$.

Case 4 One of the points $Q_{i}$, say $Q_{1}$, lies on a $\gamma$-edge and the other lies on a $\beta$-edge. Let $u_{i}, i=1, \ldots, 6$ denote the number of intersection points of $\alpha$ with the corresponding piece of $\partial$ on Figure 9. Then $u_{3}+u_{4}=u_{5}+u_{6}=|\alpha \cap \beta|=m$ and $u_{1}+u_{2} \leq m$. Also $\left|\delta_{1} \cap \alpha\right|=u_{1}+u_{4},\left|\delta_{5} \cap \alpha\right|=u_{1}+u_{3},\left|\delta_{6} \cap \alpha\right|=u_{2}+u_{5}$ and $\left|\delta_{7} \cap \alpha\right|=u_{2}+u_{6}$. Moreover, since $P_{2}$ and $Q_{2}$ are connected by an arc of $\alpha$, they represent different points on $S$ (otherwise it would be the only arc of $\alpha)$ and $u_{4} \neq u_{6}$. It follows that $\left|\delta_{i} \cap \alpha\right|<m$ for $i=1,5,6$ or 7 .

We may assume now that for every pair of $\operatorname{arcs}(i, j)$ whose end-points separate each other no two end-points lie on the same $\beta$-edge. If $P_{i}$ and $Q_{i}$ lie on a $\gamma$-edge and $P_{j}$ lie in between then $a_{i}$ together with the interval of $\gamma$ between $P_{i}$ and $Q_{i}$ form a nonseparating curve which meets $\alpha$ less than $m$ times. So we may assume that $P_{1}$ and $P_{2}$ lie on different $\beta$-edges, say $P_{1}$ on the left edge and $P_{2}$ on the right edge, and $Q_{1}$ and $Q_{2}$ lie on the lower edge. Replacing $Q_{1}$ or $Q_{2}$ by an intermediate point, if necessary, we may also assume that for every point $Q_{i}$ between $Q_{1}$ and $Q_{2}$ the corresponding point $P_{i}$ also lies between $Q_{1}$ and $Q_{2}$. Now if there is no point of $\alpha$ on the left edge below $P_{1}$ then $\left|\delta_{1} \cap \alpha\right|<m$. If there is such point of $\alpha$ consider the one closest to $P_{1}$ and call it $P_{3}$. Then, by our assumptions, point $Q_{3}$ lies on the lower edge to the left of $Q_{2}$ and $\left|\delta_{8} \cap \alpha\right|<m$.

This concludes the proof of the Lemma.

Proposition 19 A path $\mathbf{p}$ of radius $m$ around $\alpha$ is null-homotopic.

Proof Let $v_{0}$ be a vertex of $\mathbf{p}$ containing $\alpha$. We say that $\mathbf{p}$ begins at $v_{0}$. Let $v_{1}$ be the first vertex of $\mathbf{p}$ which has distance $m$ from $\alpha$. Let $\mathbf{q}$ be the 

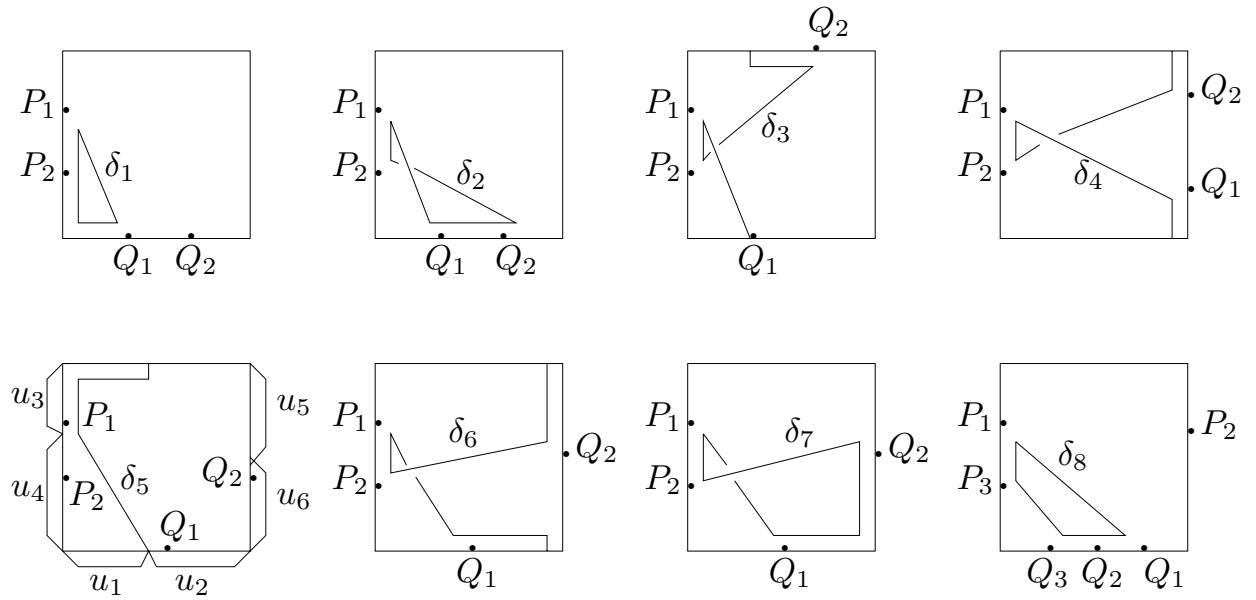

Figure 9: Constructing curve $\delta$

maximal segment of $\mathbf{p}$, which starts at $v_{1}$ and contains some fixed curve $\beta$ satisfying $|\beta \cap \alpha|=m$ and such that no vertex of $\mathbf{q}$ contains a curve $\beta^{\prime}$ satisfying $\left|\beta^{\prime} \cap \alpha\right|<m$. Let $v_{2}$ be the last vertex of $\mathbf{q}$. Let $u_{1}$ be the vertex of $\mathbf{p}$ preceding $v_{1}$ and let $u_{2}$ be the vertex of $\mathbf{p}$ following $v_{2}$. Vertex $u_{1}$ contains a curve $\gamma_{1}$ such that $\left|\gamma_{1} \cap \alpha\right|<m$. Vertex $u_{2}$ is the first vertex of the second segment which has a fixed curve $\gamma_{2}$ such that $\left|\gamma_{2} \cap \alpha\right| \leq m$. If $u_{1}$ contains $\beta$ then $\left|\gamma_{1} \cap \beta\right|=0$. Otherwise, since $v_{1}$ does not contain $\gamma_{1}$, the move from $u_{1}$ to $v_{1}$ involves $\gamma_{1}$ and $\beta$, so $\left|\gamma_{1} \cap \beta\right|=1$. If $v_{2}$ contains $\gamma_{2}$ then $\left|\gamma_{2} \cap \beta\right|=0$. It may also happen that $\left|\gamma_{2} \cap \alpha\right|<m$ and that $\beta \in u_{2}$. Then also $\left|\gamma_{2} \cap \beta\right|=0$. Otherwise $\left|\gamma_{2} \cap \beta\right|=1$. We want to construct vertices $w_{1}, w_{1}^{\prime}, w_{2}$ and $w_{2}^{\prime}$ and edge paths connecting them, as on Figure 10, so that the rectangles are null homotopic. Then we can replace the part of $\mathbf{p}$ between $u_{1}$ and $u_{2}$ by the path connecting consecutively $u_{1}$ to $w_{1}^{\prime}, w_{1}^{\prime}$ to $w_{1}, w_{1}$ to $w_{2}, w_{2}$ to $w_{2}^{\prime}$ and $w_{2}^{\prime}$ to $u_{2}$. We denote the new path by $\mathbf{p}^{\prime}$.

In our construction vertex $w_{i}$ contains a nonseparating curve $\delta_{i}$ disjoint from $\beta$. If $\left|\gamma_{i} \cap \beta\right|=0$ we let $\delta_{i}=\gamma_{i}, w_{i}=w_{i}^{\prime}=u_{i}$ and the corresponding rectangle degenerates to an edge. If $\left|\gamma_{i} \cap \beta\right|=1$ we proceed as follows. By Lemma 18 there exists a nonseparating curve $\delta_{i}$ such that $\left|\delta_{i} \cap \beta\right|=0,\left|\delta_{i} \cap \alpha\right|<m$, and $\left|\delta_{i} \cap \gamma_{i}\right| \leq 1$. If $\left|\delta_{i} \cap \gamma_{i}\right|=0$ (this is always the case if $m=1$ ) then $\left[\delta_{i}\right] \neq\left[\gamma_{i}\right]$ and $\left[\delta_{i}\right] \neq[\beta]$ because they have different intersections. There exists a vertex $w_{i}^{\prime}$ containing $\delta_{i}$ and $\gamma_{i}$ and a vertex $w_{i}$ containing $\delta_{i}$ and $\beta$. We can connect $u_{i}$ to $w_{i}^{\prime}$ by a $\gamma_{i}$-segment, $w_{i}^{\prime}$ to $w_{i}$ by a $\delta_{i}$-segment and $w_{i}$ to $v_{i}$ by a $\beta$-segment. The corresponding rectangle has radius zero around $\delta_{i}$, so it is null-homotopic 
by the Induction Hypothesis 2 .

If $\left|\delta_{i} \cap \gamma_{i}\right|=1$ there exist vertices $w_{i}^{\prime}$ and $w_{i}$ which are connected by an edge and contain $\gamma_{i}$ and $\delta_{i}$ respectively. We connect $w_{i}^{\prime}$ to $u_{i}$ by a $\gamma_{i}$-segment. We now apply Lemma 17 to vertices $w_{i}$ and $v_{i}$ with $\delta_{1}, \delta_{2}, \alpha, \beta$ replaced by $\delta_{i}, \beta, \gamma_{i}, \beta$ respectively and $m>1$. There exists a path connecting $w_{i}$ to $v_{i}$ such that all vertices of the path have distance less than $m$ from $\gamma_{i}$ and $\beta$. The corresponding rectangle has radius less than $m$ around $\gamma_{i}$ so it is nullhomotopic, by the Induction Hypothesis 2.

We now apply Lemma 17 to vertices $w_{1}$ and $w_{2}$. There exists a path $\mathbf{q}=$ $\left(w_{1}=z_{1}, z_{2}, \ldots, z_{k}=w_{2}\right)$ connecting $w_{1}$ and $w_{2}$ such that $d\left(z_{i}, \beta\right)<m$ for all $i, d\left(z_{i}, \alpha\right)<m$ for $1 \leq i \leq j<k$ and $z_{i}$ contains $\delta_{2}$ for $j<i \leq k$. In particular the middle rectangle on Figure 10 has radius less than $m$ around $\beta$ so it is null-homotopic by the Induction Hypothesis 2 . All vertices of the new part of path $\mathbf{p}^{\prime}$ have distance less than $m$ from $\alpha$ except for the final $\gamma_{2}$-segment from $w_{2}^{\prime}$ to $u_{2}$, if $\left|\gamma_{2} \cap \beta\right|=1$, or final $\delta_{2}=\gamma_{2}$-segment of $\mathbf{q}$, if $\left|\gamma_{2} \cap \beta\right|=0$ and the right rectangle degenerates. Thus $\mathbf{p}^{\prime}$ has smaller number of segments at the distance $m$ from $\alpha$, it has no $\beta$-segment, and it is null-homotopic by induction on the number of segments of $\mathbf{p}$ at the distance $m$ from some curve $\alpha$.

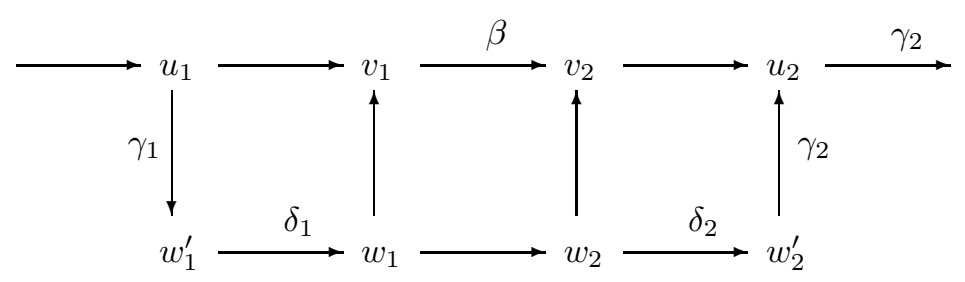

Figure 10: Reducing a path of radius $m$

This concludes the proof of Theorem 4 .

\section{A presentation of $M_{g, 1}$}

In this section we shall consider a surface $S$ of genus $g>1$ with one boundary component $\partial$. Let $\mathcal{M}_{g, 1}$ be the mapping class group of $S$. Let $X$ be the cut-system complex of $S$ described in the previous section. We shall establish a presentation of $\mathcal{M}_{g, 1}$ via its action on $X$. The action of $\mathcal{M}_{g, 1}$ on $X$ is 
defined by its action on vertices of $X$. If $v=\left\langle C_{1}, \ldots, C_{g}\right\rangle$ is a vertex of $X$ and $g \in \mathcal{M}_{g, 1}$ then $g(v)=\left\langle g\left(C_{1}\right), \ldots, g\left(C_{g}\right)\right\rangle$.

We start with some properties of homeomorphisms of a surface. Then we describe stabilizers of vertices and edges of the action of $\mathcal{M}_{g, 1}$ on $X$. Finally we consider the orbits of faces of $X$ and determine a presentation of $X$.

In order to shorten some long formulas we shall adopt the following notation for conjugation: $a * b=a b a^{-1}$. As usually $[a, b]=a b a^{-1} b^{-1}$.

Remark 3 Some proofs of relations between homeomorphisms of a surface are left to the reader. The general idea of a proof is as follows. We split the surface into a union of disks by a finite number of curves (and arcs with the end-points on the boundary if the surface has a boundary). We prove that the given product of homeomorphisms takes each curve (respectively arc) onto an isotopic curve (arc), preserving some fixed orientation of the curve (arc). Then the product is isotopic to a homeomorphism pointwise fixed on each curve and arc. But a homeomorphism of a disk fixed on its boundary is isotopic to the identity homeomorphism, relative to the boundary, by Lemma of Alexander. Thus the given product of homeomorphisms is isotopic to the identity.

Dehn proved in [5] that every homeomorphism of $S$ is isotopic to a product of twists. We start with some properties of twists.

Lemma 20 Let $\alpha$ be a curve on $S$, let $h$ be a homeomorphism and let $\alpha^{\prime}=$ $h(\alpha)$. Then $T_{\alpha^{\prime}}=h T_{\alpha} h^{-1}$.

Proof Since $h$ maps $\alpha$ to $\alpha^{\prime}$ we may assume that (up to isotopy) it also maps a neighborhood $N$ of $\alpha$ to a neighborhood $N^{\prime}$ of $\alpha^{\prime}$. The homeomorphism $h^{-1}$ takes $N^{\prime}$ to $N$, then $T_{\alpha}$ maps $N$ to $N$, twisting along $\alpha$, and $h$ takes $N$ back to $N^{\prime}$. Since $T_{\alpha}$ is supported in $N$, the composite map is supported in $N^{\prime}$ and is a Dehn twist about $\alpha^{\prime}$.

Lemma 21 Let $\gamma_{1}, \gamma_{2}, \ldots, \gamma_{k}$ be a chain of curves, ie, the consecutive curves intersect once and non-consecutive curves are disjoint. Let $N$ denote the regular neighbourhood of the union of these curves. Let $c_{i}$ denote the twist along $\gamma_{i}$. Then the following relations hold:

(i) The "commutativity relation": $c_{i} c_{j}=c_{j} c_{i}$ if $|i-j|>1$.

(ii) The "braid relation": $c_{i} c_{j}\left(\gamma_{i}\right)=\gamma_{j}$, and $c_{i} c_{j} c_{i}=c_{j} c_{i} c_{j}$ if $|i-j|=1$. 
(iii) The "chain relation": If $k$ is odd then $N$ has two boundary components, $\partial_{1}$ and $\partial_{2}$, and $\left(c_{1} c_{2} \ldots c_{k}\right)^{k+1}=T_{\partial_{1}} T_{\partial_{2}}$. If $k$ is even then $N$ has one boundary component $\partial_{1}$ and $\left(c_{1} c_{2} \ldots c_{k}\right)^{2 k+2}=T_{\partial_{1}}$.

(iv) $\left(c_{2} c_{1} c_{3} c_{2}\right)\left(c_{4} c_{3} c_{5} c_{4}\right)\left(c_{2} c_{1} c_{3} c_{2}\right)=\left(c_{4} c_{5} c_{3} c_{4}\right)\left(c_{2} c_{1} c_{3} c_{2}\right)\left(c_{4} c_{3} c_{5} c_{4}\right)$.

(v) $\left(c_{1} c_{2} \ldots c_{k}\right)^{k+1}=\left(c_{1} c_{2} \ldots c_{k-1}\right)^{k}\left(c_{k} c_{k-1} \ldots c_{2} c_{1}^{2} c_{2} \ldots c_{k-1} c_{k}\right)=$ $\left(c_{k} c_{k-1} \ldots c_{2} c_{1}^{2} c_{2} \ldots c_{k-1} c_{k}\right)\left(c_{1} c_{2} \ldots c_{k-1}\right)^{k}$.

Proof Relation (i) is obvious. It follows immediately from the definition of Dehn twist that $c_{2}\left(\gamma_{1}\right)=c_{1}^{-1}\left(\gamma_{2}\right)$. Both statements of (ii) follow from this and from Lemma 20. Relation (iii) is a little more complicated. It can be proven by the method explained in Remark 3. Relations (iv) and (v) follow from the braid relations (i) and (ii) by purely algebraic operations. We shall prove (iv).

$$
\begin{aligned}
& \left(c_{2} c_{1} c_{3} c_{2}\right)\left(c_{4} c_{3} c_{5} c_{4}\right)\left(c_{2} c_{1} c_{3} c_{2}\right)=c_{2} c_{3} c_{1} c_{2} c_{4} c_{5} c_{3} c_{4} c_{2} c_{3} c_{1} c_{2}= \\
& c_{2} c_{3} c_{4} c_{1} c_{5} c_{2} c_{3} c_{2} c_{4} c_{3} c_{1} c_{2}=c_{2} c_{3} c_{4} c_{3} c_{1} c_{5} c_{2} c_{3} c_{4} c_{3} c_{1} c_{2}= \\
& c_{4} c_{2} c_{3} c_{4} c_{1} c_{5} c_{2} c_{4} c_{3} c_{1} c_{2} c_{4}=c_{4} c_{2} c_{3} c_{1} c_{4} c_{5} c_{4} c_{2} c_{3} c_{1} c_{2} c_{4}= \\
& c_{4} c_{5} c_{2} c_{3} c_{1} c_{4} c_{2} c_{3} c_{1} c_{2} c_{5} c_{4}=c_{4} c_{5} c_{2} c_{3} c_{1} c_{2} c_{1} c_{4} c_{3} c_{2} c_{5} c_{4}= \\
& c_{4} c_{5} c_{3} c_{2} c_{3} c_{4} c_{1} c_{2} c_{3} c_{2} c_{5} c_{4}=c_{4} c_{5} c_{3} c_{2} c_{3} c_{4} c_{3} c_{1} c_{2} c_{3} c_{5} c_{4}= \\
& c_{4} c_{5} c_{3} c_{4} c_{2} c_{3} c_{4} c_{1} c_{2} c_{3} c_{5} c_{4}=\left(c_{4} c_{5} c_{3} c_{4}\right)\left(c_{2} c_{1} c_{3} c_{2}\right)\left(c_{4} c_{3} c_{5} c_{4}\right) .
\end{aligned}
$$

We now prove (v). We prove by induction that for $s \leq k$ we have $\left(c_{1} c_{2} \ldots c_{k}\right)^{s}=$ $\left(c_{1} c_{2} \ldots c_{k-1}\right)^{s}\left(c_{k} c_{k-1} \ldots c_{k-s+1}\right)$. Using (i) and (ii) one checks easily that for $i>1$ we have $c_{i}\left(c_{1} c_{2} \ldots c_{k}\right)=\left(c_{1} c_{2} \ldots c_{k}\right) c_{i-1}$. Now

$\left(c_{1} c_{2} \ldots c_{k}\right)^{s+1}=\left(c_{1} c_{2} \ldots c_{k-1}\right)^{s}\left(c_{k} c_{k-1} \ldots c_{k-s+1}\right)\left(c_{1} c_{2} \ldots c_{k}\right)=$ $\left(c_{1} c_{2} \ldots c_{k-1}\right)^{s} c_{1} c_{2} \ldots c_{k} c_{k-1} \ldots c_{k-s}=\left(c_{1} c_{2} \ldots c_{k-1}\right)^{s+1} c_{k} c_{k-1} \ldots c_{k-s}$.

For $s=k+1$ we get $\left(c_{1} c_{2} \ldots c_{k}\right)^{k+1}=\left(c_{1} c_{2} \ldots c_{k-1}\right)^{k}\left(c_{k} c_{k-1} \ldots c_{1} c_{1} c_{2} \ldots c_{k}\right)$.

This proves the first equality in (v). By (i) and (ii) $\left(c_{k} c_{k-1} \ldots c_{2} c_{1}^{2} c_{2} \ldots c_{k-1} c_{k}\right)$ commutes with $c_{i}$ for $i<k$, which implies the second equality.

The next lemma was observed by Dennis Johnson in [12] and was called a lantern relation.

Lemma 22 Let $U$ be a disk with the outer boundary $\partial$ and with 3 inner holes bounded by curves $\partial_{1}, \partial_{2}, \partial_{3}$ which form vertices of a triangle in the clockwise order. For $1 \leq i<j \leq 3$ let $\alpha_{i, j}$ be the simple closed curve in $U$ which bounds a neighbourhood of the "edge" $\left(\partial_{i}, \partial_{j}\right)$ of the triangle (see Figure 11). Let $d$ be the twist along $\partial, d_{i}$ the twist along $\partial_{i}$ and $a_{i, j}$ the twist along $\alpha_{i, j}$.

Then $d d_{1} d_{2} d_{3}=a_{1,2} a_{1,3} a_{2,3}$. 


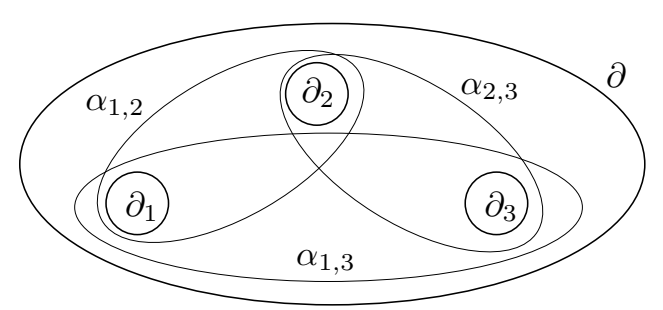

Figure 11: Lantern relation

We now describe a presentation of the mapping class group of a disk with holes.

Lemma 23 Let $U$ be a disk with the outer boundary $\partial$ and with $n$ inner holes bounded by curves $\partial_{1}, \partial_{2}, \ldots, \partial_{n}$. For $1 \leq i<j \leq n$ let $\alpha_{i, j}$ be the simple closed curve in $U$ shown on Figure 12, separating two holes $\partial_{i}$ and $\partial_{j}$ from the other holes. Let $d$ be the twist along $\partial, d_{i}$ the twist along $\partial_{i}$ and $a_{i, j}$ the twist along $\alpha_{i, j}$. Then the mapping class group of $U$ has a presentation with generators $d_{i}$ and $a_{i, j}$ and with relations

(Q1) $\left[d_{i}, d_{j}\right]=1$ and $\left[d_{i}, a_{j, k}\right]=1$ for all $i, j, k$.

(Q2) pure braid relations

(a) $a_{r, s}^{-1} * a_{i, j}=a_{i, j}$ if $r<s<i<j$ or $i<r<s<j$,

(b) $a_{r, s}^{-1} * a_{s, j}=a_{r, j} * a_{s, j}$ if $r<s<j$,

(c) $a_{r, j}^{-1} * a_{r, s}=a_{s, j} * a_{r, s}$ if $r<s<j$,

(d) $\left[a_{i, j}, a_{r, j}^{-1} * a_{r, s}\right]=1$ if $r<i<s<j$.

Proof Relations (Q2) come in place of standard relations in the pure braid group on $n$ strings and we shall first prove the equivalence of (Q2) to the standard presentation of the pure braid group. The standard presentation has generators $a_{i, j}$ and relations (this is a corrected version of the relations in [2]):

(i) $a_{r, s}^{-1} * a_{i, j}=a_{i, j}$ if $r<s<i<j$ or $i<r<s<j$,

(ii) $a_{r, s}^{-1} * a_{s, j}=a_{r, j} * a_{s, j}$ if $r<s<j$,

(iii) $\left[a_{r, j}, a_{s, j}\right]=\left[a_{r, s}^{-1}, a_{r, j}^{-1}\right]$ if $r<s<j$,

(iv) $a_{r, s}^{-1} * a_{i, j}=\left[a_{r, j}, a_{s, j}\right] * a_{i, j}$ if $r<i<s<j$.

So relations (a) and (b) are the same as (i) and (ii) respectively. We can substitute relation (iii) in (iv) and get (d), after cancellation of $a_{r, s}$. When we 


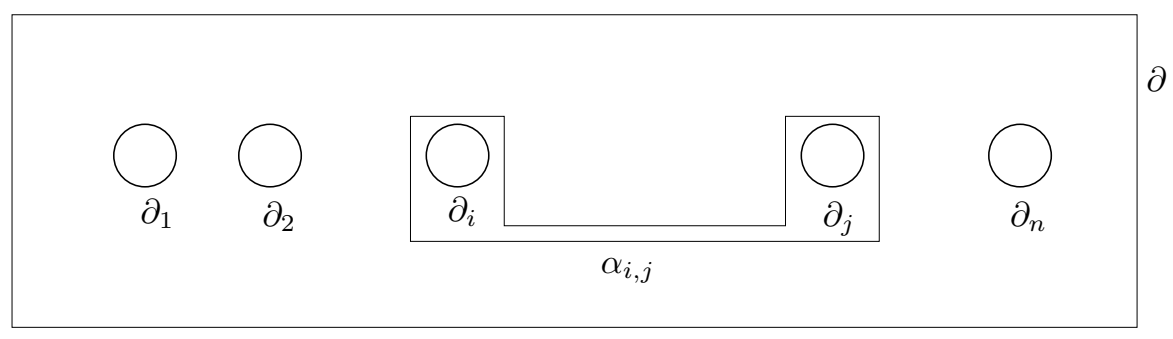

Figure 12: Generators of $\mathcal{M}_{0, n}$

substitute (ii) for the first three terms of (iii) we get (c), after cancellation of $a_{r, s}^{-1}$.

We now consider the disk $U$ with holes. When we glue a disk with a distinguished center to each curve $\partial_{i}$ we get a disk with $n$ distinquished points. Its mapping class group is isomorphic to the pure braid group $P_{n}$ with generators $a_{i, j}$ and with relations (Q2). In the passage from the mapping class group of $U$ to the mapping class group of the punctured disk we kill exactly the twists $d_{i}$, which commute with everything. One can check that the removal of the disks does not affect the relations (Q2) so the mapping class group of $U$ has a presentation with relations (Q1) and (Q2).

We now consider the surface $S$. We shall fix some curves on $S$. The surface $S$ consists of a disk with $g$ handles attached to it. For $i=1, \ldots, g$ and $j=$ $1, \ldots, g-1$ we fix curves $\alpha_{i}, \beta_{i}, \epsilon_{j}$ (see Figure 1). Curve $\alpha_{i}$ is a meridian curve across the $i$-th handle, $\beta_{i}$ is a curve along the $i$-th handle and $\epsilon_{i}$ runs along $i$-th handle and $(1+i)$-th handle. Curves $\alpha_{1}, \alpha_{2}, \ldots, \alpha_{g}$ form a cut-system.

We denote by $I_{0}$ the set of indices $I_{0}=\{-g, 1-g, 2-g, \ldots,-1,1,2, \ldots, g-1, g\}$. When we cut $S$ open along the curves $\alpha_{1}, \ldots, \alpha_{g}$ we get a disk $S_{0}$ with $2 g$ holes bounded by curves $\partial_{i}, i \in I_{0}$, where curves $\partial_{i}$ and $\partial_{-i}$ correspond to the same curve $\alpha_{i}$ on $S$ (see Figure 13). The glueing back map identifies $\partial_{i}$ with $\partial_{-i}$ according to the reflection with respect to the $x$-axis. Curves on $S$ can be represented on $S_{0}$. If a curve on $S$ meets some curves $\alpha_{i}$ then it is represented on $S_{0}$ by a disjoint union of several arcs. In particular $\epsilon_{i}$ is represented by two arcs joining $\partial_{-i}$ to $\partial_{-i-1}$ and $\partial_{i}$ to $\partial_{i+1}$. We denote by $\delta_{i, j}, i<j \in I_{0}$, curves on $S$ represented on Figure 13. Curve $\delta_{i, j}$ separates holes $\partial_{i}$ and $\partial_{j}$ from the other holes on $S_{0}$. We now fix some elements of $\mathcal{M}_{g, 1}$.

Definition 4 We denote by $a_{i}, b_{i}, e_{j}$ the Dehn twists along the curves $\alpha_{i}, \beta_{i}, \epsilon_{j}$ respectively. We fix the following elements of $\mathcal{M}_{g, 1}$ : 
$s=b_{1} a_{1} a_{1} b_{1}$.

$t_{i}=e_{i} a_{i} a_{i+1} e_{i}$ for $i=1, \ldots, g-1$.

$d_{1,2}=\left(b_{1}^{-1} a_{1}^{-1} e_{1}^{-1} a_{2}^{-1}\right) * b_{2}$.

For $i<j \in I_{0}$ we let

$d_{i, j}=\left(t_{i-1} t_{i-2} \ldots t_{1} t_{j-1} t_{j-2} \ldots t_{2}\right) * d_{1,2}$ if $i>0$,

$d_{i, j}=\left(t_{-i-1}^{-1} t_{-i-2}^{-1} \ldots t_{1}^{-1} s^{-1} t_{j-1} t_{j-2} \ldots t_{2}\right) * d_{1,2}$ if $i<0$ and $i+j>0$,

$d_{i, j}=\left(t_{-i-1}^{-1} t_{-i-2}^{-1} \ldots t_{1}^{-1} s^{-1} t_{j} t_{j-1} \ldots t_{2}\right) * d_{1,2}$ if $i<0, j>0$ and $i+j<0$,

$d_{i, j}=\left(t_{-j-1}^{-1} t_{-j-2}^{-1} \ldots t_{1}^{-1} t_{-i-1}^{-1} t_{-i-2}^{-1} \ldots t_{2}^{-1} s^{-1} t_{1}^{-1} s^{-1}\right) * d_{1,2}$ if $j<0$,

$d_{i, j}=\left(t_{j-1}^{-1} d_{j-1, j} t_{j-2}^{-1} d_{j-2, j-1} \ldots t_{1}^{-1} d_{1,2}\right) *\left(s^{2} a_{1}^{4}\right)$ if $i+j=0$.

The products described in the above definition represent very simple elements of $\mathcal{M}_{g, 1}$ and we shall explain their meaning now. We shall first define special homeomorphisms of $S_{0}$.

Definition 5 A half-twist $h_{i, j}$ along a curve $\delta_{i, j}$ is an isotopy class (on $S_{0}$ relative to its boundary) of a homeomorphism of $S_{0}$ which is fixed outside $\delta_{i, j}$ and is equal to a counterclockwise "rotation" by 180 degrees inside $\delta_{i, j}$. In particular $h_{i, j}$ switches the two holes $\partial_{i}$ and $\partial_{j}$ inside $\delta_{i, j}$ so it is not fixed on the boundary of $S_{0}$, but $h_{i, j}^{2}$ is fixed on the boundary of $S_{0}$ and is isotopic to the full Dehn twist along $\delta_{i, j}$.

Lemma 24 The result of the action of $t_{k}$ (respectively $s$ ) on a curve $\delta_{i, j}$ is the same as the result of the action of the product of half-twists $h_{k, k+1} h_{-k-1,-k}$ (respectively the result of the action of $h_{-1,1}$ ) on $\delta_{i, j}$. So $t_{k}$ rotates $\delta_{i, j}$ around $\delta_{k, k+1}$ counterclockwise and around $\delta_{-k-1,-k}$ conterclockwise and switches the corresponding holes. If the pair $(i, j)$ is disjoint from $\{k, k+1,-k,-k-1\}$ than $t_{k}$ leaves $\delta_{i, j}$ fixed. It also leaves curves $\delta_{k, k+1}$ and $\delta_{-k-1,-k}$ fixed. In a similar way $s$ rotates $\delta_{i, j}$ counterclockwise around $\delta_{-1,1}$ and switches the holes. If $(i, j)$ is disjoint from $(-1,1)$ than $s$ leaves $\delta_{i, j}$ fixed. Also $\delta_{-1,1}$ is fixed by $s$.

Proof The result of the action can be checked directly.

Lemma 25 The element $d_{i, j}$ of $\mathcal{M}_{g, 1}$ is equal to the twist along the curve $\delta_{i, j}$ for all $i, j$.

Proof We start with the curve $\beta_{2}$ and apply to it the product of twists $b_{1}^{-1} a_{1}^{-1} e_{1}^{-1} a_{2}^{-1}$. We get the curve $\delta_{1,2}$. Therefore, by Lemma $20, d_{1,2}$ is equal 
to the twist along $\delta_{1,2}$. For $i \neq-j$ we start with $\delta_{1,2}$ and apply consecutive factors $t_{i}$ and $s$, one at a time. We check that the result is $\delta_{i, j}$ so $d_{i, j}$ is the twist along $\delta_{i, j}$, by Lemma 20. For $d_{-1,1}$ we use (iii) of Lemma 21. The curve $\delta_{-1,1}$ is the boundary of a regular neighbourhood of $\alpha_{1} \cup \beta_{1}$ and $\left(a_{1} b_{1}\right)^{6}=s^{2} a_{1}^{4}$ by (i) and (ii) of Lemma 21, therefore $d_{-1,1}=\left(a_{1} b_{1}\right)^{6}$ is the twist along $\delta_{-1,1}$ by (iii) of Lemma 21. Now we apply a suitable product of $t_{i}$ 's and $d_{i, i+1}$ 's to $\delta_{-1,1}$ and get $\delta_{-j, j}$. Therefore $d_{-j, j}$ is equal to the twist along $\delta_{-j, j}$ by Lemma 20 .

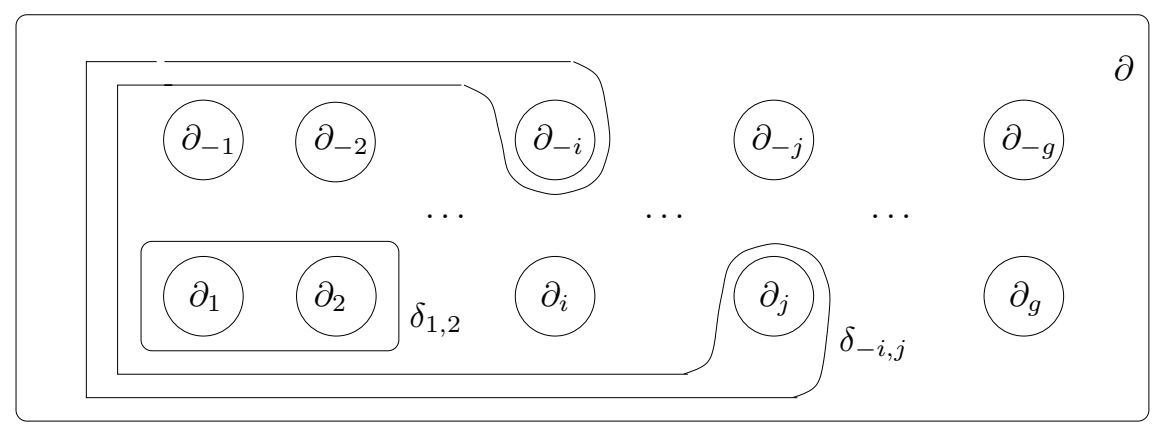

Figure 13: Curves $\delta_{i, j}$ on $S_{0}$

We can explain now the relations in Theorem 1.

Lemma 26 The relations (M1), (M2) and (M3) from Theorem 1 are satisfied in $\mathcal{M}_{g, 1}$.

Proof Relations (M1) follow from Lemma 21 (i) and (ii). Curves $\beta_{1}, \alpha_{1}, \epsilon_{1}$ form a chain. One boundary component of a regular neighbourhood of $\alpha_{1} \cup \beta_{1} \cup$ $\epsilon_{1}$ is equal to $\beta_{2}$. It is easy to check that $a_{2} e_{1} a_{1} b_{1}^{2} a_{1} e_{1} a_{2}\left(\beta_{2}\right)$ is equal to the other boundary component. By Lemma 21 (iii) and by Lemma 20 we have $\left(b_{1} a_{1} e_{1}\right)^{4}=$ $b_{2} a_{2} e_{1} a_{1} b_{1}^{2} a_{1} e_{1} a_{2} b_{2}\left(a_{2} e_{1} a_{1} b_{1}^{2} a_{1} e_{1} a_{2}\right)^{-1}$. This is equivalent to relation (M2) by Lemma $21(\mathrm{v})$. Consider now relation (M3). Applying consecutive twists one can check that $\left(b_{2} a_{2} e_{1} b_{1}^{-1}\right)\left(\delta_{1,3}\right)=\delta_{3}$, where $\delta_{3}$ is the curve on Figure 1 . Thus $d_{3}$ represents the twist along $\delta_{3}$. When we cut $S$ along curves $\alpha_{1}, \alpha_{2}, \alpha_{3}$ and $\delta_{3}$ we split off a sphere with four holes from surface $S$. Since elements $d_{i, j}$ represent twists along curves $\delta_{i, j}$, relation (M3) follows from lantern relation, Lemma 22.

Our first big task is to establish a presentation of a stabilizer of one vertex of $X$. Let $v_{0}$ be a fixed vertex of $X$ corresponding to the cut system $\left\langle\alpha_{1}, \alpha_{2}, \ldots, \alpha_{g}\right\rangle$. Let $H$ be the stabilizer of $v_{0}$ in $\mathcal{M}_{g, 1}$. 
Proposition 27 The stabilizer $H$ of vertex $v_{0}$ admits the following presentation:

The set of generators consists of $a_{1}, a_{2}, \ldots, a_{g}, s, t_{1}, t_{2}, \ldots, t_{g-1}$ and the $d_{i, j}$ 's for $i<j, \quad i, j \in I_{0}$.

The set of defining relations consists of:

(P1) $\left[a_{i}, a_{j}\right]=1$ and $\left[a_{i}, d_{j, k}\right]=1$ for all $i, j, k \in I_{0}$.

(P2) pure braid relations

(a) $d_{r, s}^{-1} * d_{i, j}=d_{i, j}$ if $r<s<i<j$ or $i<r<s<j$,

(b) $d_{r, s}^{-1} * d_{s, j}=d_{r, j} * d_{s, j}$ if $r<s<j$,

(c) $d_{r, j}^{-1} * d_{r, s}=d_{s, j} * d_{r, s}$ if $r<s<j$,

(d) $\left[d_{i, j}, d_{r, j}^{-1} * d_{r, s}\right]=1$ if $r<i<s<j$.

(P3) $t_{i} t_{i+1} t_{i}=t_{i+1} t_{i} t_{i+1}$ for $i=1, \ldots, g-2$ and $\left[t_{i}, t_{j}\right]=1$ if $1 \leq i<$ $j-1<g-1$.

(P4) $s^{2}=d_{-1,1} a_{1}^{-4}$ and $t_{i}^{2}=d_{i, i+1} d_{-i-1,-i} a_{i}^{-2} a_{i+1}^{-2} \quad$ for $i=1, \ldots, g-1$.

(P5) $\left[t_{i}, s\right]=1$ for $i=2, \ldots, g-1$.

(P6) $s t_{1} s t_{1}=t_{1} s t_{1} s$.

(P7) $\left[s, a_{i}\right]=1$ for $1 \leq i \leq g, \quad t_{i} * a_{i}=a_{i+1}$ for $1 \leq i \leq g-1$, $\left[a_{i}, t_{j}\right]=1$ for $1 \leq i \leq g, j \neq i, i-1$.

(P8) $s * d_{i, j}=d_{i, j}$ if $i \neq \pm 1$ and $j \neq \pm 1$ or if $i=-1$ and $j=1$, $s * d_{-1, j}=d_{1, j}$ for $2 \leq j \leq g, \quad s * d_{i,-1}=d_{i, 1} \quad$ for $\quad-g \leq i \leq-2$, $t_{k} * d_{i, j}=d_{i, j}$ if $1 \leq k \leq g-1$ and $(j=i+1=k+1$, or $j=i+1=-k$ or $i, j \notin\{ \pm k, \pm(k+1)\})$,

$t_{k} * d_{k, j}=d_{k+1, j}$ for $1 \leq k \leq g-1$ and $k+2 \leq j \leq g$,

$t_{k} * d_{i,-k-1}=d_{i,-k}$ for $1 \leq k \leq g-1$ and $-g \leq i \leq-k-2$,

$t_{k} * d_{-k-1, k}=d_{-k, k+1}, \quad t_{k} * d_{-k-1, k+1}=d_{k, k+1} * d_{-k, k}$, for $1 \leq k \leq g-1$

$t_{k} * d_{-k-1, j}=d_{-k, j}$ for $1 \leq k \leq g-1$ and $j>-k, j \neq k, k+1$,

$t_{k} * d_{i, k}=d_{i, k+1}$ for $1 \leq k \leq g-1$ and $i<k, \quad i \neq-k,-k-1$.

Proof An element of $H$ leaves the cut-system $v_{0}$ invariant but it may permute the curves $\alpha_{i}$ and may reverse their orientation. Clearly $a_{i}$ belongs to $H$. One can easily check that $t_{i}\left(\alpha_{i}\right)=\alpha_{i+1}, t_{i}\left(\alpha_{i+1}\right)=\alpha_{i}, t_{i}\left(\alpha_{k}\right)=\alpha_{k}$ for $k \neq i, i+1$. 
$s\left(\alpha_{1}\right)$ is equal to $\alpha_{1}$ with the opposite orientation and $s$ is fixed on other $\alpha_{i}$ 's. Thus $t_{i}$ 's and $s$ belong to $H$. By Lemma 25 we know that $d_{i, j}$ is a twist along the curve $\delta_{i, j}$ so it also belongs to $H$. We shall prove in the next section that the relations (P1) - (P8) follow from the relations (M1) - (M3), so they are satisfied in $\mathcal{M}_{g, 1}$ and thus also in $H$. The group $H$ can be defined by two exact sequences.

$$
\begin{aligned}
& 1 \rightarrow \mathbb{Z}_{2}^{g} \rightarrow \pm \Sigma_{g} \rightarrow \Sigma_{g} \rightarrow 1 . \\
& 1 \rightarrow H_{0} \rightarrow H \rightarrow \pm \Sigma_{g} \rightarrow 1 .
\end{aligned}
$$

Before defining the objects and the homomorphisms in these sequences we shall recall the following fact from group theory.

Lemma 28 Let $1 \rightarrow A \rightarrow B \rightarrow C \rightarrow 1$ be an exact sequence of groups with known presentations $A=\left\langle a_{i} \mid Q_{j}\right\rangle$ and $C=\left\langle c_{i} \mid W_{j}\right\rangle$. A presentation of $B$ can be obtained as follows: Let $b_{i}$ be a lifting of $c_{i}$ to $B$. Let $R_{j}$ be a word obtained from $W_{j}$ by substitution of $b_{i}$ for each $c_{i}$. Then $R_{j}$ represents an element $d_{j}$ of $A$ which we write as a product of generators $a_{i}$ of $A$. Finally for every $a_{i}$ and $b_{j}$ the conjugate $b_{j} * a_{i}$ represents an element $a_{i, j}$ of $A$, which we write as a product of the generators $a_{i}$.

Then $B=\left\langle a_{i}, b_{j} \mid Q_{j}, R_{j}=d_{j}, b_{j} * a_{i}=a_{i, j}\right\rangle$.

We now describe the sequence (1) and the group $\pm \Sigma_{g}$. This is the group of permutations of the set $I_{0}=\{-g, 1-g, \ldots,-1,1,2, \ldots, g\}$ such that $\sigma(-i)=$ $-\sigma(i)$. The homomorphism $\pm \Sigma_{g} \rightarrow \Sigma_{g}$ forgets the signs. A generator of the kernel changes the sign of one letter. The sequence splits, $\Sigma_{g}$ can be considered as the subgroup of the permutations which take positive numbers to positive numbers. Let $\tau_{i}=(i, i+1)$ be a transposition in $\Sigma_{g}$ for $i=1,2, \ldots, g-1$. Then

(S1) $\left[\tau_{i}, \tau_{j}\right]=1$ for $|i-j|>1$,

(S2) $\tau_{i} * \tau_{i+1}=\tau_{i+1}^{-1} * \tau_{i}$ for $i=1, \ldots, g-2$,

(S3) $\tau_{i}^{2}=1$ for $i=1, \ldots, g-1$.

This defines a presentation of $\Sigma_{g}$. Further let $\sigma_{i}$ for $i=1, \ldots, g$ denote the change of sign of the $i$-th letter in a signed permutation. Then $\sigma_{i}^{2}=1$ and $\left[\sigma_{i}, \sigma_{j}\right]=1$ for all $i, j$. Finally the conjugation gives $\tau_{i} * \sigma_{i}=\sigma_{i+1}, \tau_{i} * \sigma_{i+1}=\sigma_{i}$ and $\left[\sigma_{j}, \tau_{i}\right]=1$ for $j \neq i$ and $j \neq i+1$. In fact it suffices to use one generator $\sigma=\sigma_{1}$ and the relations $\sigma_{i}=\left(\tau_{i-1} \tau_{i-2} \ldots \tau_{1}\right) * \sigma$. We get the relations

(S4) $\sigma^{2}=1$, 
(S5) $\left[\sigma, \tau_{j}\right]=1$ and $\left[\left(\tau_{i} \tau_{i-1} \ldots \tau_{1}\right) * \sigma, \tau_{j}\right]=1$ for $1 \leq i \leq g-1$ and $j \neq i$ and $j \neq i+1$,

(S6) $\left[\left(\tau_{i} \tau_{i-1} \ldots \tau_{1}\right) * \sigma, \sigma\right]=1$ and $\left[\left(\tau_{i} \tau_{i-1} \ldots \tau_{1}\right) * \sigma,\left(\tau_{j} \tau_{j-1} \ldots \tau_{1}\right) * \sigma\right]=1$ for $1 \leq i, j \leq g-1$.

Group $\pm \Sigma_{g}$ has a presentation with generators $\sigma, \tau_{1}, \ldots, \tau_{g-1}$ and with defining relations $(\mathrm{S} 1)-(\mathrm{S} 6)$.

We shall describe now the sequence (2). A homeomorphism in $H$ may permute the curves $\alpha_{i}$ and may change their orientations. We fix an orientation of each curve $\alpha_{i}$ and define a homomorphism $\phi_{1}: H \rightarrow \pm \Sigma_{g}$ as follows: a homeomorphism $h$ is mapped onto a permutation $i \mapsto \pm j$ if $h\left(\alpha_{i}\right)=\alpha_{j}$ and the sign is "+" if the orientations of $h\left(\alpha_{i}\right)$ and of $\alpha_{j}$ agree, and "-" otherwise. If $h$ preserves the isotopy class of $\alpha_{i}$ and preserves its orientation then it is isotopic to a homeomorphism fixed on $\alpha_{i}$. The kernel of $\phi_{1}$ is the subgroup $H_{0}$ of the elements of $H$ represented by the homeomorphisms which keep the curves $\alpha_{1}, \alpha_{2}, \ldots, \alpha_{g}$ pointwise fixed. We want to find a presentation of $H$ from the sequence (2). We start with a presentation of $H_{0}$. An element of $H_{0}$ induces a homeomorphism of $S_{0}$. When we glue back the corresponding pairs of boundary components of $S_{0}$ we get the surface $S$. This glueing map induces a homomorphism from the mapping class group of $S_{0}$ onto $H_{0}$.

We shall prove that the kernel of this homomorphism is generated by products $d_{i} d_{-i}^{-1}$, where $d_{i}$ is the twist along curve $\partial_{i}$, so both twists are identified with $a_{i}$ in $H_{0}$. It suffices to assume, by induction, that we glue only one pair of boundaries $\partial_{i}$ and $\partial_{-i}$ on $S_{0}$ and get a nonseparating curve $\alpha_{i}$ on $S$. Homeomorphism $d_{i} d_{-i}^{-1}$ induces a spin map $s$ of $S$ along $\alpha_{i}$ (see [2], Theorem 4.3 and Fig. 14). Let $\gamma$ be a curve on $S$ which meets $\alpha_{i}$ at one point $P$. Let $h_{0}$ be a homeomorphism of $S_{0}$ which induces a homeomorphism $h$ of $S$ isotopic to the identity and fixed on $\alpha_{i}$. We shall prove that for a suitable power $k$ the map $s^{k} h$ is fixed on $\gamma$ (after an isotopy of $S$ liftable to $S_{0}$ ). If $h(\gamma)$ forms a 2 -gon with $\gamma$ we can get rid of the 2 -gon by an isotopy liftable to $S_{0}$ (fixed on $\left.\alpha_{i}\right)$. If $h(\gamma)$ and $\gamma$ form no 2-gons then they are tangent at $P$. Let us move $h(\gamma)$ off $\gamma$, near the point $P$, to a curve $\gamma^{\prime}$. If $\gamma^{\prime}$ and $\gamma$ intersect then they form a 2-gon. But then $h(\gamma)$ and $\gamma$ form a self-touching 2-gon (see Figure 14). Clearly the spin map $s$ or $s^{-1}$ removes the 2-gon. If $\gamma^{\prime}$ and $\gamma$ are disjoint then they bound an annulus. If the annulus contains the short arc of $\alpha_{i}$ between $\gamma^{\prime}$ and $\gamma$ then $h(\gamma)$ is isotopic to $\gamma$ by an isotopy liftable to $S_{0}$. If the annulus contains the long arc of $\alpha_{i}$ between $\gamma^{\prime}$ and $\gamma$ then the situation is similar to Figure 14, but $h(\gamma)$ does not meet $\gamma$ outside $P$. The spin map $s$ or $s^{-1}$ takes $h(\gamma)$ onto a curve isotopic to $\gamma$ by an isotopy liftable to $S_{0}$. So we may 
assume, by induction on $|h(\gamma) \cap \gamma|$, that $h(\gamma)=\gamma$. We proceed as in Remark 3. We spilt $S$ into disks by curves $\alpha_{i}, \gamma$, and other curves disjoint from $\alpha_{i}$. Homeomorphism $h$ takes each curve to an isotopic curve and the 2-gons which appear do not contain $\alpha_{i}$. Thus all isotopies are liftable to $S_{0}$.

It follows that the kernel is generated by $d_{i} d_{-i}^{-1}$ 's. By Lemma 23 the map-

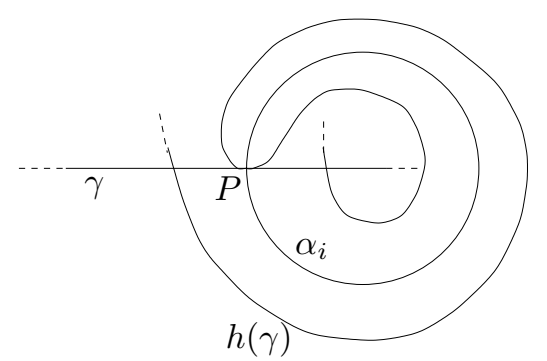

Figure 14: Self-touching 2-gon

ping class group of $S_{0}$ has a presentation with generators $d_{k}$ and $d_{i, j}$ and with relations (Q1) and (Q2), where $a_{i, j}=d_{i, j}, i, j \in I_{0}$. Therefore $H_{0}$ has a presentation with generators $a_{1}, \ldots, a_{g}$ and $d_{i, j}$ for $i<j \in I_{0}$ and with relations (P1), (P2). In these relations $d_{i, j}$ is represented by a Dehn twist along $\delta_{i, j}$.

We come back to sequence (2). We see from the action of $t_{i}$ and $s$ on $\alpha_{j}$ that we can lift $\tau_{i}$ to $t_{i}$ and $\sigma$ to $s$. Relations (S1) and (S2) lift to (P3). Relations (S3) and (S4) lift to (P4). Relation (S5) lifts to $\left[\left(t_{i} t_{i-1} \ldots t_{1}\right) * s, t_{j}\right]=1$ for $j \neq i$ and $j \neq i+1$ and it follows from (P3) and (P5). We shall deal with (S6) a little later. We now pass to the conjugation of the generators of $H_{0}$ by $s$ and $t_{k}$. Since $s^{2} \in H_{0}$ and $t_{k}^{2} \in H_{0}$, by (P4), it suffices to know the result of the conjugation of each generator of $H_{0}$ by either $s$ or by $s^{-1}$, and by either $t_{k}$ or by $t_{k}^{-1}$, the other follows. The result of the conjugation is described in relations (P7) and (P8). Finally we lift the relation (S6). We start with the case $\left[\tau_{1} * \sigma, \sigma\right]$. It lifts to $t_{1} s t_{1}^{-1} s t_{1} s^{-1} t_{1}^{-1} s^{-1}=($ by $(\mathrm{P} 6)) t_{1} s t_{1}^{-1} s s^{-1} t_{1}^{-1} s^{-1} t_{1}=t_{1} s t_{1}^{-2} s^{-1} t_{1}^{-1} t_{1}^{2}$. We have $t_{1}^{2} \in H_{0}$, by (P4), and the conjugation of an element of $H_{0}$ by $s$ and $t_{i}$ is already determined by $(\mathrm{P} 7)$ and $(\mathrm{P} 8)$. So we know how to lift $\left[\tau_{1} * \sigma, \sigma\right]=1$. In the general case we have a commutator $\left[\left(t_{i} t_{i-1} \ldots t_{1}\right) * s,\left(t_{j} t_{j-1} \ldots t_{1}\right) * s\right]$. If $i>j$ then, by (P3) and (P5), this commutator is equal to the conjugate of $\left[t_{1} * s, s\right]$ by $t_{j} t_{j-1} \ldots t_{1} t_{i} t_{i-1} \ldots t_{2}$. This is a conjugation of an element of $H_{0}$ by $t_{k}$ 's, so the result is determined by $(\mathrm{P} 7)$ and $(\mathrm{P} 8)$.

This concludes the proof of Proposition 27.

We shall prove now that $\mathcal{M}_{g, 1}$ acts transitively on vertices of $X$, so there is only one vertex orbit, and that $H$ acts transitively on edges incident to $v_{0}$, so 
there is only one edge orbit. When we cut $S$ open along the curves $\alpha_{1}, \ldots, \alpha_{g}$ we get a disk with $2 g$ holes. It is homeomorphic to any other such disk and we can prescribe the homeomorphism on the boundary components, hence every two cut systems can be transformed into each other by a homeomorphism and $\mathcal{M}_{g, 1}$ acts transitively on $X^{0}$. Let $w$ be a vertex connected to $v_{0}$ by an edge. Then $w$ contains a curve $\beta$ which intersects some curve $\alpha_{i}$ at one point and is disjoint from the other curves of $v_{0}$. We cut $S$ open along $\alpha_{1}, \alpha_{2}, \ldots, \alpha_{g}, \beta$ and get a disk with $2 g-1$ holes, including one "big" hole bounded by arcs of both $\alpha_{i}$ and $\beta$. If we cut $S$ along curves belonging to another edge incident to $v_{0}$ we get a similar situation. There exists a homeomorphism of one disk with $2 g-1$ holes onto the other which preserves the identification of curves of the boundary and takes the set $\alpha_{1}, \alpha_{2}, \ldots, \alpha_{g}$ onto itself. It induces a homeomorphism of $S$ which leaves $v_{0}$ invariant and takes one edge onto the other. Thus $H$ acts transitively on the edges incident to $v_{0}$.

We now fix one such edge and describe its stabilizer. If we replace curve $\alpha_{1}$ of cut-system $v_{0}$ by curve $\beta_{1}$ we get a new cut-system connected to $v_{0}$ by an edge. We denote the cut-system by $v_{0}^{\prime}$ and the edge by $\mathbf{e}_{0}$. Let $H^{\prime}$ be the stabilizer of $\mathbf{e}_{0}$ in $H$.

Lemma 29 The stabilizer $H^{\prime}$ of the edge $\mathbf{e}_{0}$ is generated by $a_{1}^{2} s, t_{1} s t_{1}, a_{2}$, $d_{2,3}, d_{-2,2}, d_{-1,1} d_{-1,2} d_{1,2} a_{1}^{-2} a_{2}^{-1}$, and $t_{2}, \ldots, t_{g-1}$.

Proof An element $h$ of $H^{\prime}$ may permute curves $\alpha_{2}, \ldots, \alpha_{g}$ and may reverse their orientations. It may also reverse simultaneously orientations of both curves $\alpha_{1}$ and $\beta_{1}$ (it preserves the orientation of $S$ so it must preserve algebraic intersection of curves). We check that $a_{1}^{2} s$ reverses orientations of $\beta_{1}$ and $\alpha_{1}$, $t_{1} s t_{1}$ reverses orientation of $\alpha_{2}$ and leaves $\beta_{1}$ and $\alpha_{1}$ invariant. Elements $t_{i}$ permute the curves $\alpha_{2}, \ldots, \alpha_{g}$. Modulo these homeomorphisms we may assume that $h$ is fixed on all curves $\alpha_{i}$ and $\beta_{1}$. It induces a homeomorphism of $S$ cut open along all these curves. We get a disk with holes and, by Lemma 23, its mapping class group is generated by twists around holes and twists along suitable curves surrounding two holes at a time. Element $d_{-1,1}=\left(a_{1}^{2} s\right)^{2}$ represents twist around the big hole (the cut along $\alpha_{1} \cup \beta_{1}$ ). Conjugates of $a_{2}$ by $t_{j}$ 's produce twists around other holes. The conjugate of $d_{2,3}$ by $\left(t_{1} s t_{1}\right)^{-1}$ is equal to $d_{-2,3}$ and the conjugate of $d_{-2,3}$ by $\left(t_{2} t_{1} s t_{1}\right)^{-1}$ is equal to $d_{-3,-2}$. Every $d_{k, k+1}$ can be obtained from these by conjugation by $t_{i}$ 's, $i>1$. It is now clear that every curve $\delta_{i, j}$ with $i, j \neq \pm 1$ can be obtained from $d_{2,3}$ and $d_{-2,2}$ by conjugation by the elements chosen in the Lemma. So the corresponding twists are products of the above generators. We also need twists along curves 
which surround the "big" hole and another hole. Consider such a curve on $S_{0}$. It must contain inside both holes $\partial_{-1}$ and $\partial_{1}$, including the arc between them corresponding to $\beta_{1}$, and one other hole. One such curve, call it $\gamma$, contains $\partial_{-1}, \partial_{1}, \partial_{2}$. By Lemma 22 the twist along $\gamma$ is equal to $d_{-1,1} d_{-1,2} d_{1,2} a_{1}^{-2} a_{2}^{-1}$. Any other curve which contains $\partial_{-1}, \partial_{1}, \partial_{i}$ with $i>1$ is obtained from $\gamma$ by application of $t_{i}$ 's. The curve which contains $\partial_{-1}, \partial_{1}, \partial_{-2}$ is obtained from $\gamma$ by application of $\left(t_{1} s t_{1}\right)^{-1}$ and a curve which contains $\partial_{-1}, \partial_{1}, \partial_{i}$ with $i<-2$ is obtained from the last curve by application of $t_{i}^{-1}$ 's. Therefore all generators of $H^{\prime}$ are products of the generators in the Lemma.

We now distinguish one more element of $\mathcal{M}_{g, 1}$, which does not belong to $H$. Let $r=a_{1} b_{1} a_{1}$. The element $r$ is a "quarter-twist". It switches curves $\alpha_{1}$ and $\beta_{1}, r^{2}=s a_{1}^{2}=h_{-1,1}$ is a half-twist around $\delta_{-1,1}$ and $r^{4}=d_{-1,1}$. Also $r$ leaves the other curves $\alpha_{i}$ fixed, so it switches the vertices of the edge $\mathbf{e}_{0}, r\left(v_{0}\right)=v_{0}^{\prime}$ and $r\left(v_{0}^{\prime}\right)=v_{0}$.

We now describe precisely a construction from [15] and [9] which will let us determine a presentation of $\mathcal{M}_{g, 1}$. This construction was very clearly explained in [10].

Let us consider a free product $(H * \mathbb{Z})$ where the group $\mathbb{Z}$ is generated by $r$. An $h$-product is an element of $(H * \mathbb{Z})$ with positive powers of $r$, so it has a form $h_{1} r h_{2} r \ldots h_{k} r h_{k+1}$. We have an obvious map $\eta:(H * \mathbb{Z}) \rightarrow \mathcal{M}_{g, 1}$ through which the $h$-products act on $X$. We shall prove that $\eta$ is onto and we shall find the $h$ products which normally genarate the kernel of $\eta$.

To every edge-path $\mathbf{p}=\left(v_{0}, v_{1}, \ldots, v_{k}\right)$ which begins at $v_{0}$ we assign an $h-$ product $g=h_{1} r h_{2} r \ldots h_{k} r h_{k+1}$ such that $h_{1} r \ldots h_{m} r\left(v_{0}\right)=v_{m}$ for $m=$ $1, \ldots, k$. We construct it as follows. There exists $h_{1} \in H$ such that $h_{1}\left(v_{0}\right)=v_{0}$ and $h_{1}\left(v_{0}^{\prime}\right)=v_{1}$. Then $h_{1} r\left(v_{0}\right)=v_{1}$. Next we transport the second edge to $v_{0}$. $\left(h_{1} r\right)^{-1}\left(v_{1}\right)=v_{0}$ and $\left(h_{1} r\right)^{-1}\left(v_{2}\right)=v_{1}^{\prime}$. There exists $h_{2} \in H$ such that $h_{2} r\left(v_{0}\right)=v_{1}^{\prime}$ and $h_{1} r h_{2} r\left(v_{0}\right)=v_{2}$. And so on. Observe that the elements $h_{i}$ in the $h$-product corresponding to an edge-path $\mathbf{p}$ are not uniquely determined. In particular $h_{k+1}$ is an arbitrary element of $H$. The construction implies:

Lemma 30 The generators of $H$ together with the element $r$ generate the group $\mathcal{M}_{g, 1}$.

Proof Let $g$ be an element of $\mathcal{M}_{g, 1}$. Then $g\left(v_{0}\right)$ is a vertex of $X$ and can be connected to $v_{0}$ by an edge-path $\mathbf{p}=\left(v_{0}, v_{1}, \ldots, v_{k}=g\left(v_{0}\right)\right)$. Let $g_{1}=$ $h_{1} r h_{2} r \ldots h_{k} r$ be an $h$-product corresponding to $\mathbf{p}$. Then $g_{1}\left(v_{0}\right)=v_{k}=g\left(v_{0}\right)$, therefore $\eta\left(g_{1}^{-1}\right) g=h_{k+1}$ leaves $v_{0}$ fixed and belongs to the stabilizer $H$ of $v_{0}$. It follows that $g=\eta\left(h_{1} r h_{2} r \ldots h_{k} r h_{k+1}\right)$ in $\mathcal{M}_{g, 1}$. 
By the inverse process we define an edge-path induced by the $h$-product $g=$ $h_{1} r h_{2} r \ldots h_{k} r h_{k+1}$. The edge-path starts at $v_{0}$ then $v_{1}=h_{1} r\left(v_{0}\right), v_{2}=$ $h_{1} r h_{2} r\left(v_{0}\right)$ and so on. The last vertex of the path is $v_{k}=g\left(v_{0}\right)$.

Remark 4 An $h$-product $g$ represents an element in $H$ if and only if $v_{k}=v_{0}$. This happens if and only if the corresponding edge-path is closed. We can multiply such $g$ by a suitable element of $H$ on the right and get an $h$-product which represents the identity in $\mathcal{M}_{g, 1}$ and induces the same edge-path as $g$.

We now describe a presentation of $\mathcal{M}_{g, 1}$.

Theorem 31 The mapping class group $\mathcal{M}_{g, 1}$ admits the following presentation:

The set of generators consists of $a_{1}, a_{2}, \ldots, a_{g}, s, t_{1}, t_{2}, \ldots, t_{g-1}, r$ and the $d_{i, j}$ 's for $i<j, \quad i, j \in I_{0}$.

The set of defining relations consists of relations $(\mathrm{P} 1)-(\mathrm{P} 8)$ of Proposition 27 and of the following relations:

(P9) $r$ commutes with $a_{1}^{2} s, t_{1} s t_{1}, a_{2}, d_{2,3}, d_{-2,2}, d_{-1,1} d_{-1,2} d_{1,2} a_{1}^{-2} a_{2}^{-1}$, and $t_{2}, \ldots, t_{g-1}$.

(P10) $r^{2}=s a_{1}^{2}$.

(P11) $\left(k_{i} r\right)^{3}=\left(k_{i} s a_{1}\right)^{2}$ for $i=1,2,3,4$, where

$k_{1}=a_{1}, k_{2}=d_{1,2}, k_{3}=a_{1}^{-1} a_{2}^{-2} d_{1,2} d_{-2,1} d_{-2,2}, k_{4}=a_{1}^{-1} a_{2}^{-1} a_{3}^{-1} d_{1,2} d_{1,3} d_{2,3}$, $\left(r k_{5} r k_{5}^{-1}\right)^{2}=s a_{1}^{2} k_{5} s a_{1}^{2} k_{5}^{-1}$, where $k_{5}=a_{2} t_{1} d_{1,2}^{-1},\left(r a_{1} t_{1}\right)^{5}=\left(s a_{1}^{2} t_{1}\right)^{4}$.

Relations (P9) - (P11) say that some elements of $H * \mathbb{Z}$ belong to $\operatorname{ker}(\eta)$, so Theorem 31 claims that $(H * \mathbb{Z})$ modulo relations $(\mathrm{P} 9)-(\mathrm{P} 11)$ is isomorphic to $\mathcal{M}_{g, 1}$.

Relations (P9) tell us that $r$ commutes with the generators of the stabilizer $H^{\prime}$ of the edge $\mathbf{e}_{0}$. We shall prove this claim now. An element $h$ of $H^{\prime}$ leaves the edge $\mathbf{e}_{0}$ fixed but it may reverse the orientations of $\alpha_{1}$ and $\beta_{1}$. The element $r^{2}=s a_{1}^{2}$ does exactly this and commutes with $r$. Modulo this element we may assume that $h$ leaves $\alpha_{1}$ and $\beta_{1}$ pointwise fixed. But then we may also assume that it leaves some neighbourhood of $\alpha_{1}$ and $\beta_{1}$ pointwise fixed. On the other hand $r$ is equal to the identity outside a neighbourhood of $\alpha_{1}$ and $\beta_{1}$ so it commutes with $h$.

From relations (P9) and (P10) we get information about $h$-products.

Claim 1 If two $h$-products represent the same element in $\mathcal{M}_{g, 1}$ and induce the same edge-path then they are equal in $(H * \mathbb{Z}) /(P 9)$. 
Proof If two $h$-products $g_{1}=h_{1} r \ldots r h_{k+1}$ and $g_{2}=f_{1} r \ldots r f_{k+1}$ induce the same edge-path $\mathbf{p}=\left(v_{0}, v_{1}, \ldots, v_{k}\right)$ then $h_{1} r\left(v_{0}\right)=f_{1} r\left(v_{0}\right)$. Therefore $h_{1}^{-1} f_{1} r\left(v_{0}\right)=r\left(v_{0}\right)$ and $h_{1}^{-1} f_{1} \in H^{\prime}$ commutes with $r$ in $(H * \mathbb{Z}) /(P 9)$. Now $f_{1} r f_{2}=h_{1} h_{1}^{-1} f_{1} r f_{2}=h_{1} r f_{2}^{\prime}$ in $(H * \mathbb{Z}) /(P 9)$. Therefore $g_{2}$ and a new $h_{-}$ product $h_{1} r f_{2}^{\prime} r f_{3} r \ldots r f_{k+1}$ are equal in $(H * \mathbb{Z}) /(P 9)$ and induce the same edge-path $\mathbf{p}$. If we apply $r^{-1} h_{1}^{-1}$ to the vertices $\left(v_{1}, v_{2}, \ldots, v_{k}\right)$ of $\mathbf{p}$ we get a shorter edge-path which starts at $v_{0}$ and is induced by both shorter $h$-products $h_{2} r \ldots r h_{k+1}$ and $f_{2}^{\prime} r \ldots r f_{k+1}$. Claim 1 follows by induction on $k$.

Two different edge-paths may be homotopic in the 1-skeleton $X^{1}$. This means that there is a backtracking $v_{i}, v_{i+1}, v_{i}$ along the edge-path.

Claim 2 If two $h$-products represent the same element in $\mathcal{M}_{g, 1}$ and induce edge-paths which are equal modulo back-tracking then the $h$-products are equal in $(H * \mathbb{Z}) /((P 9),(P 10))$.

Proof Consider an $h$-product $g=g_{i} h_{i+1} r h_{i+2} r$, where $g_{i}$ is an $h$-product inducing a shorter edge-path $\mathbf{p}$ and the edge-path induced by $g$ has a backtracking at the end: $g_{i}\left(v_{0}\right)=v_{i}, g_{i} h_{i+1} r\left(v_{0}\right)=v_{i+1}$, and $g_{i} h_{i+1} r h_{i+2} r\left(v_{0}\right)=$ $v_{i}$. Clearly the $h$-product $g_{i} h_{i+1} r r$ induces the same edge-path. In particular $g_{i} h_{i+1} r^{2}\left(v_{0}\right)=v_{i}$ hence there exists $h^{\prime} \in H$ such that $\eta\left(g_{i} h_{i+1} r^{2} h^{\prime}\right)=$ $\eta\left(g_{i} h_{i+1} r h_{i+2} r\right)$. Now by Claim 1 the $h$-products are equal in $(H * \mathbb{Z}) /(P 9)$. But $g_{i} h_{i+1} r^{2} h^{\prime}$ is equal in $(H * \mathbb{Z}) /(P 10)$ to a shorter $h$-product which induces the edge-path p. Claim 2 follows by induction on the number of backtrackings.

Relations (P11) correspond to edge-paths of type (C3), (C4) and (C5). Six relations correspond to six particular edge-paths.

As in (P11) we let $k_{1}=a_{1}, \quad k_{2}=d_{1,2}, \quad k_{3}=d_{1,2} d_{-2,1} d_{-2,2} a_{2}^{-2} a_{1}^{-1}$, $k_{4}=a_{1}^{-1} a_{2}^{-1} a_{3}^{-1} d_{1,2} d_{1,3} d_{2,3}=d_{3}, \quad k_{5}=a_{2} d_{1,2}^{-1} t_{1}, \quad k_{6}=a_{1} t_{1}$.

We now choose six $h$-products. For $i=1,2,3,4$ let $g_{i}=\left(k_{i} r\right)^{3}, g_{5}=$ $\left(r k_{5} r k_{5}^{-1}\right)^{2}$ and $g_{6}=\left(r k_{6}\right)^{5}$. Product $g_{i}$ appears in the corresponding relation in (P11).

For $i=1, \ldots, 6$ let $\gamma_{i}$ be the corresponding curve on Figure 15, represented on surface $S_{0}\left(\gamma_{5}=\beta_{2}\right)$.

For $i=1,2,3,4$ homeomorphism $k_{i}$ leaves all curves $\alpha_{i}$ invariant. Also $k_{i} r\left(\alpha_{1}\right)=\gamma_{i}, k_{i} r\left(\gamma_{i}\right)=\beta_{1}, k_{i} r\left(\beta_{1}\right)=\alpha_{1}$. It follows that the $h$-product $g_{i}$ represents the edge path $\mathbf{p}_{i}=\left(\left\langle\alpha_{1}\right\rangle \rightarrow\left\langle\gamma_{i}\right\rangle \rightarrow\left\langle\beta_{1}\right\rangle \rightarrow\left\langle\alpha_{1}\right\rangle\right)$. 

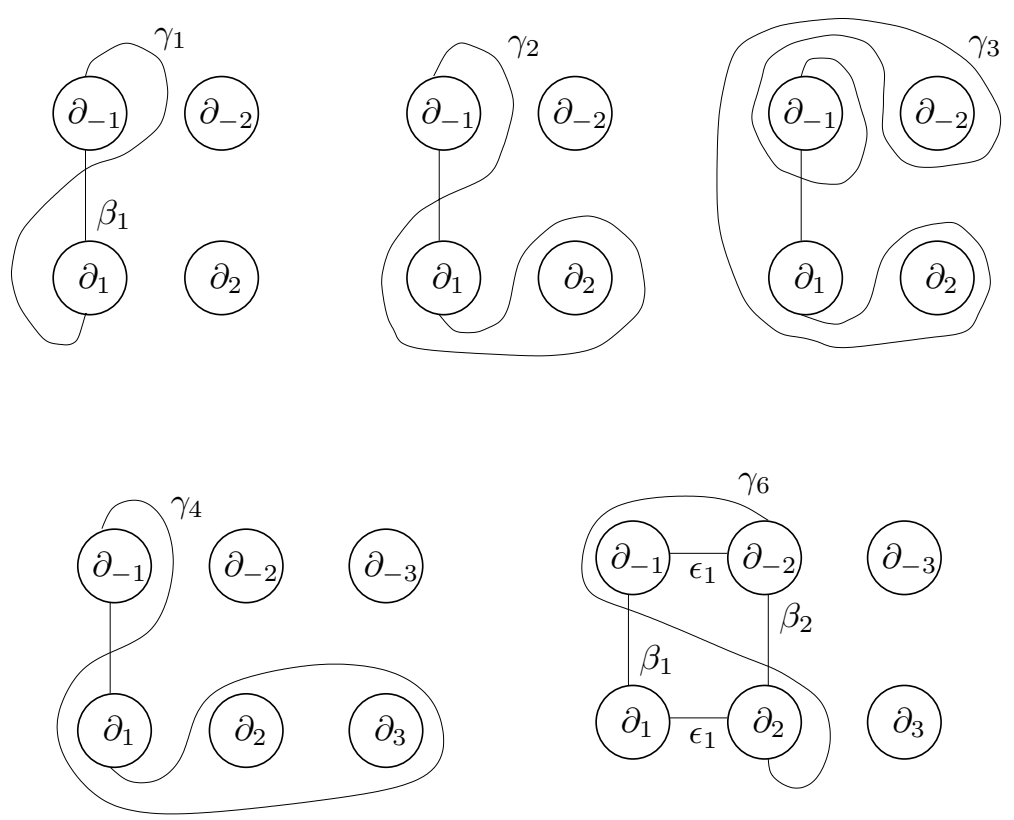

Figure 15: Curves $\gamma_{i}$

It is not hard to check that for $i=5,6$ the $h$-product $g_{i}$ represents the edgepath $\mathbf{p}_{i}$, where

$$
\begin{aligned}
& \mathbf{p}_{5}=\left(\left\langle\alpha_{1}, \alpha_{2}\right\rangle \rightarrow\left\langle\beta_{1}, \alpha_{2}\right\rangle \rightarrow\left\langle\beta_{1}, \gamma_{5}\right\rangle \rightarrow\left\langle\alpha_{1}, \gamma_{5}\right\rangle \rightarrow\left\langle\alpha_{1}, \alpha_{2}\right\rangle\right) . \\
& \mathbf{p}_{6}=\left(\left\langle\alpha_{1}, \alpha_{2}\right\rangle \rightarrow\left\langle\beta_{1}, \alpha_{2}\right\rangle \rightarrow\left\langle\beta_{1}, \epsilon_{1}\right\rangle \rightarrow\left\langle\gamma_{6}, \epsilon_{1}\right\rangle \rightarrow\left\langle\gamma_{6}, \alpha_{1}\right\rangle \rightarrow\left\langle\alpha_{2}, \alpha_{1}\right\rangle\right) .
\end{aligned}
$$

Since $g_{i}$ represents a closed edge-path it is equal in $\mathcal{M}_{g, 1}$ to some element $h_{i} \in H$. Then $V_{i}=g_{i} h_{i}^{-1}$ also represents $\mathbf{p}_{i}$ and is equal to the identity in $\mathcal{M}_{g, 1}$. We shall prove in the next section that $h_{i}$ is equal in $H$ to the right hand side of the corresponding relation in $(\mathrm{P} 11)$ and thus $V_{i}=1$ in $(H * \mathbb{Z}) /((P 9),(P 10),(P 11))$.

We already know, by Theorem 4, that every closed path in $X$ is a sum of paths of type (C3), (C4) and (C5). Some of these paths are represented by the paths $\mathbf{p}_{1}-\mathbf{p}_{6}$. We say that a closed path is conjugate to a path $\mathbf{p}_{i}$ if it has a form $\mathbf{q}_{1} \mathbf{q}_{2} \mathbf{q}_{1}^{-1}$, where $\mathbf{q}_{1}$ starts at $v_{0}$ and $\mathbf{q}_{2}$ is the image of $\mathbf{p}_{i}$ under the action of some element of $\mathcal{M}_{g, 1}$. We shall prove that every closed path is a sum of paths conjugate to one of the paths $\mathbf{p}_{1}-\mathbf{p}_{6}$ or their inverses. This will imply Theorem 31. 
We shall start with Harer's reduction for paths of type (C3) (see [7]). We fix curves $\alpha_{2}, \ldots, \alpha_{g}$ and consider cut-systems containing one additional curve. Consider a triangular path $(\langle\alpha\rangle,\langle\beta\rangle,\langle\gamma\rangle)$. Give orientations to curves $\alpha, \beta, \gamma$ so that the algebraic intersection numbers satisfy $(\alpha, \beta)=(\alpha, \gamma)=1$. Switching $\beta$ and $\gamma$, if necessary, we may also assume $(\beta, \gamma)=-1$. We cut $S$ open along $\alpha_{2}, \ldots, \alpha_{g}$ and get a torus $S_{1,2 g-1}$ with $2 g-1$ holes. Each square on Figure 16 represents a part of the universal cover of a closed torus, punctured above holes of $S_{1,2 g-1}$. We show more than one preimage of some curves in the universal cover. A fundamental region is a square bounded by $\alpha$ and $\beta$ with $2 g-1$ holes, one of them bounded by the boundary $\partial$ of $S$. We may assume that $\gamma$ crosses $\alpha$ and $\beta$ in two distinct points. Then $\gamma$ splits the square into three regions: $F_{1}$ to the right of $\gamma$ after $\gamma$ crosses $\alpha, F_{2}$ to the left of $\gamma$ after $\gamma$ crosses $\beta$, and $F_{0}$ (see Figure $16(\mathrm{a})$ ). Reversing the orientations of all curves we can switch the regions $F_{1}$ and $F_{2}$. Let $l_{i}$ be the number of holes in $F_{i}$ for $i=0,1,2$. We want to prove that every triangular path is a sum of triangular paths with $l_{1} \leq 2, l_{2}=0$, and with the hole $\partial$ not in the region $F_{1}$. We shall prove it by induction on $l_{1}+l_{2}$. A thin circle on Figure 16 denotes a single hole and a thick circle denotes all remaining holes of the region.

Suppose first that $l_{1}>1$. Move curves $\alpha, \beta, \gamma$ off itself (on a closed torus) in such a way that the region bounded by $\alpha$ and $\alpha^{\prime}$ contains only one hole, belonging to $F_{1}$, the region bounded by $\beta$ and $\beta^{\prime}$ contains another hole of $F_{1}$ and the region bounded by $\gamma$ and $\gamma^{\prime}$ contains both of these holes. Up to an isotopy (translating holes and straightening curves) the situation looks like on Figure 16 (c).

Now consider Figure 16 (b) - an octahedron projected onto one of its faces. All faces of the octahedron are "triangles" in $X$. In order to understand regions $F_{0}, F_{1}, F_{2}$ corresponding to each face we translate the fundamental domain to a suitable square in the universal cover. For every face different from $(\alpha, \beta, \gamma)$ the region $F_{1}$ has at least one hole less than $l_{1}$ and the region $F_{0}$ has all of its original holes and possibly some more. Clearly the boundary of the face $(\alpha, \beta, \gamma)$ is a sum of conjugates of the boundaries of the other faces. So by induction we may assume that $l_{1} \leq 1$. By symmetry we may assume that $l_{2} \leq 1$ and $l_{1}>2$. We chose new curves $\alpha^{\prime}, \beta^{\prime}$ and $\gamma^{\prime}$ whose liftings are shown on Figure 16 (d). Consider again the octahedron on Figure 16 (b). Now region $F_{2}$ is fixed for all faces of the octahedron and region $F_{0}$ has all of its original holes and some additional holes for all faces different from $(\alpha, \beta, \gamma)$. So by induction we may assume that $l_{1} \leq 2$ and $l_{2} \leq 1$. Suppose $l_{1}=2$ and $l_{2}=1$ and choose new curves $\alpha^{\prime}, \beta^{\prime}$ and $\gamma^{\prime}$ as on Figure $16(\mathrm{e})$. Again consider the octahedron. Now for each face different from $(\alpha, \beta, \gamma)$ at least one of the 


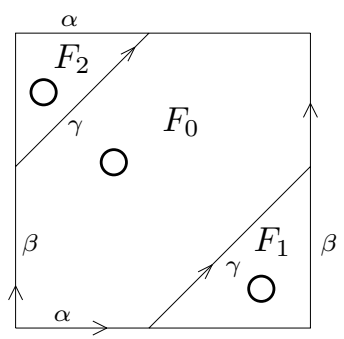

(a)

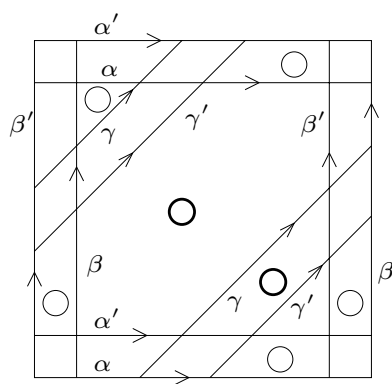

(d)

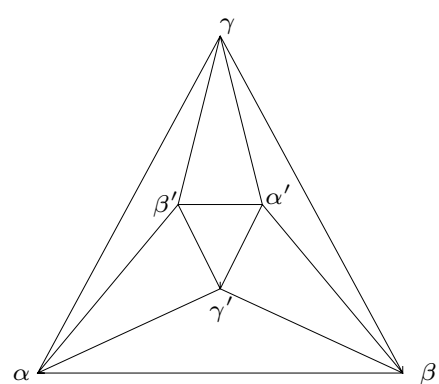

(b)

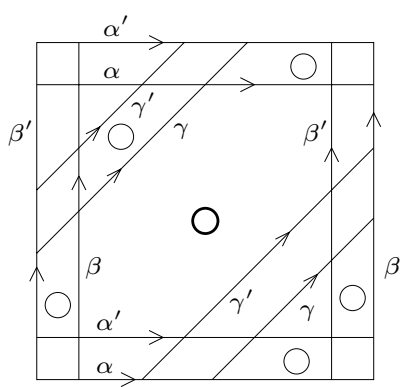

(e)

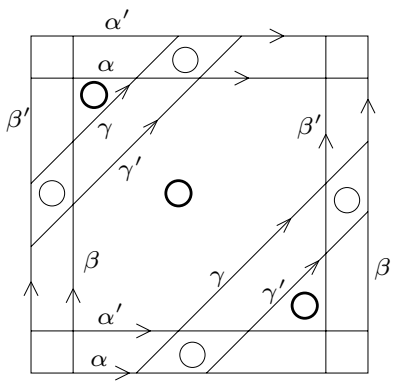

(c)

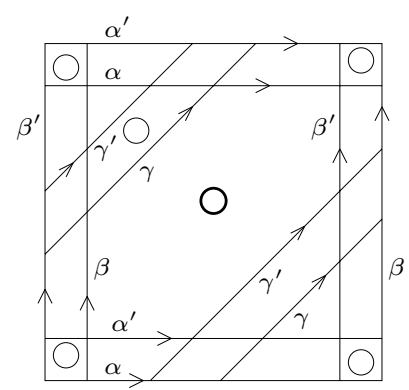

(f)

Figure 16: Reduction for paths of type (C3)

regions $F_{1}$ and $F_{2}$ looses at least one of its holes. Suppose now that each region has exactly one hole. Consider curves $\alpha^{\prime}, \beta^{\prime}$ and $\gamma^{\prime}$ on Figure 16 (f). Again for each face of the corresponding octahedron different from the face $(\alpha, \beta, \gamma)$ at least one of the regions $F_{1}$ and $F_{2}$ looses at least one of its holes. So we may assume that $l_{2}=0$ and $l_{1} \leq 2$. Finally it may happen that a hole in $F_{1}$ is bounded by $\partial$. We can isotop $\gamma$ (on the torus with holes) over $F_{2}$ to the other side of the intersection of $\alpha$ and $\beta$. Clearly $F_{1}$ becomes now $F_{0}$. We can repeat the previous reduction and the hole $\partial$ will remain in $F_{0}$. Thus we are left with four cases:

$l_{1}=l_{2}=0$,

$l_{1}=1, l_{2}=0$,

$l_{1}=2, l_{2}=0$ and the two holes in $F_{1}$ correspond to the same curve $\alpha_{i}$,

$l_{1}=2, l_{2}=0$ and the two holes in $F_{1}$ correspond to different curves $\alpha_{i}$ and $\alpha_{j}$. 
Clearly each case is uniquely determined up to homeomorphism (of one square with holes onto another, preserving $\gamma$ ). Paths $\mathbf{p}_{i}, i=1,2,3,4$ represent triangle paths of these four types with $\alpha$ corresponding to $\alpha_{1}, \beta$ corresponding to $\beta_{1}$ and $\gamma$ corresponding to $\gamma_{i}$. Given any path of type (C3) we can map it onto a path which starts at $v_{0}$. Applying an element of $H$ we may assume that the second vertex is $v_{0}^{\prime}$. Then the path is of the type considered in the above reduction and it is a sum of conjugates of $\mathbf{p}_{1}, \mathbf{p}_{2}, \mathbf{p}_{3}, \mathbf{p}_{4}$ and their inverses (we may need to switch $\beta$ and $\gamma$ in the proof).

Consider now the path $\mathbf{p}_{5}$. When we cut $S$ open along $\alpha_{1}, \alpha_{2}, \ldots, \alpha_{g}, \beta_{1}$ and $\gamma_{5}$ we get a disk with two "big" holes and $2 g-4$ "small" holes. Any other path q of type (C4) produces a similar configuration. There exists $g \in G$ which takes $\mathbf{p}_{5}$ on $\mathbf{q}$. Therefore every path of type $(\mathrm{C} 4)$ is a conjugate of $\mathbf{p}_{5}$.

Consider now $\mathbf{p}_{6}$ and another path $\mathbf{q}$ of type (C5). When we cut $S$ open along the first four changing curves of $\mathbf{p}_{6}: \alpha_{1}, \alpha_{2}, \beta_{1}, \epsilon_{1}$ and along all fixed curves of the cut systems we get a disk with one "big" hole and $2 g-4$ "small" holes. When we do the same for the curves in $\mathbf{q}$ we get a similar configuration. We may map q onto a path

$$
\left(\left\langle\alpha_{1}, \alpha_{2}\right\rangle \rightarrow\left\langle\beta_{1}, \alpha_{2}\right\rangle \rightarrow\left\langle\beta_{1}, \epsilon_{1}\right\rangle \rightarrow\left\langle\gamma, \epsilon_{1}\right\rangle \rightarrow\left\langle\gamma, \alpha_{1}\right\rangle \rightarrow\left\langle\alpha_{2}, \alpha_{1}\right\rangle\right)
$$

in which only the curve $\gamma$ is different from the corresponding curve $\gamma_{6}$ in $\mathbf{p}_{6}$ and all other curves are as in $\mathbf{p}_{6}$. Consider curve $\gamma_{5}=\beta_{2}$ on Figure 15. Curve $\gamma_{5}$ can be homotop onto the union of $\beta_{1}, \epsilon_{1}$ and a part of $\alpha_{1}$. Since $\gamma$ intersects $\beta_{1}$ once and does not intersect $\alpha_{1}$ nor $\epsilon_{1}$ it must intersect $\gamma_{5}$ once. Therefore we may form a subgraph of $X$ as on Figure 17 .

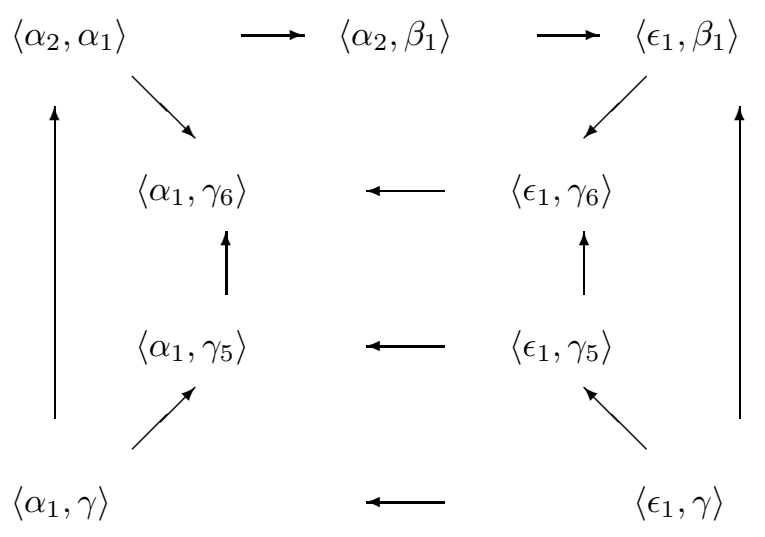

Figure 17: Reduction for paths of type (C5) 
Here bottom and middle square edge-paths are of type (C4). In the square edge-path on the left side (and on the right side) only one curve changes so the path is a sum of triangles by Proposition 7. The top pentagon edge-path is equal to $\mathbf{p}_{6}$. The new edge-path coincides with the outside pentagon. It is a sum of conjugates of the other edge paths.

We can now complete the proof of Theorem 31. Let $W \in H * \mathbb{Z}$ be such that $\eta(W)=1$. We want to prove that $W=1$ in $(H * \mathbb{Z}) /((P 9),(P 10),(P 11))$. Modulo (P10) we can write $W$ as an $h$-product $g$ which represents a closed edge path p. By Theorem 4 the edge-path $\mathbf{p}$ is a sum of conjugates of path of type (C3), (C4) and (C5). By the above discussion it is a sum of conjugates of the special paths $\mathbf{p}_{1}-\mathbf{p}_{6}$ and their inverses, modulo backtracking. So, modulo backtracking, $\mathbf{p}=\Pi \mathbf{q}_{i} f_{i}\left(\mathbf{p}_{j_{i}}^{ \pm 1}\right) \mathbf{q}_{i}^{-1}$ for some $f_{i} \in \mathcal{M}_{g, 1}$. Each path $\mathbf{q}_{i} f_{i}\left(\mathbf{p}_{j_{i}}^{ \pm 1}\right) \mathbf{q}_{i}^{-1}$ can be represented by an $h$-product $g_{i}$. Since the path is closed we have $\eta\left(g_{i}\right) \in H$, so we can correct the $h$-product $g_{i}$ and assume that $\eta\left(g_{i}\right)=1$. The product $\Pi g_{i}$ represents the path $\Pi \mathbf{q}_{i} f_{i}\left(\mathbf{p}_{j_{i}}^{ \pm 1}\right) \mathbf{q}_{i}^{-1}$, so, by Claims 1 and $2, W$ is equal to $\Pi g_{i}$ in $(H * \mathbb{Z}) /((P 9),(P 10))$. It suffices to prove that $g_{i}=1$ in $(H * \mathbb{Z}) /((P 9),(P 10),(P 11))$. Let $k=j_{i}$ and suppose that $g_{i}$ represents a path $\mathbf{q}_{i} f_{i}\left(\mathbf{p}_{k}\right) \mathbf{q}_{i}^{-1}$. Let $u_{i}$ be an $h$-product representing $\mathbf{q}_{i}$. Then $u_{i}\left(v_{0}\right)=f_{i}\left(v_{0}\right)$ is the first vertex of $f_{i}\left(\mathbf{p}_{k}\right)$, hence $\eta\left(u_{i}\right)^{-1} f_{i}=h_{i} \in H$. Recall that $\mathbf{p}_{k}$ can be represented by an $h$-product $V_{k}$ which is equal to the identity in $(H * \mathbb{Z}) /((P 9),(P 10),(P 11))$. The $h$-product $u_{i} h_{i} V_{k}$ represents $\mathbf{q}_{i} f_{i}\left(\mathbf{p}_{k}\right)$ and there exists an $h$-product $u_{i} h_{i} V_{k} w_{i}$ such that $\eta\left(u_{i} h_{i} V_{i} w_{i}\right)=1$ and $u_{i} h_{i} V_{k} w_{i}$ represents the edge-path $\mathbf{q}_{i} f_{i}\left(\mathbf{p}_{k}\right) \mathbf{q}_{i}^{-1}$. Clearly $u_{i} h_{i} w_{i}$ represents the edge-path $\mathbf{q}_{1} \mathbf{q}_{1}^{-1}$ which is null-homotopic by backtracking. By Claims 1 and 2 the $h-$ product $g_{i}$ is equal to $u_{i} h_{i} V_{k} w_{i}$ in $(H * \mathbb{Z}) /((P 9),(P 10)), u_{i} h_{i} V_{k} w_{i}$ is equal to $u_{i} h_{i} w_{i}$ in $(H * \mathbb{Z}) /((P 9),(P 10),(P 11))$ and $u_{i} h_{i} w_{i}=1$ in $(H * \mathbb{Z}) /((P 9),(P 10))$.

The path inverse to $\mathbf{p}_{k}$ is represented by some other $h$-product $V_{k}^{\prime}$ but then $V_{k} V_{k}^{\prime}$ represents a path contractible by back-tracking. Thus $V_{k} V_{k}^{\prime}=1$ in $(H *$ $\mathbb{Z}) /((P 9),(P 10))$ and $V_{k}=1$ in $(H * \mathbb{Z}) /((P 9),(P 10),(P 11))$ hence also $V_{k}^{\prime}=1$

in $(H * \mathbb{Z}) /((P 9),(P 10),(P 11))$.

This concludes the proof of Theorem 31 .

\section{Reduction to a simple presentation}

Let us recall the following obvious direction of Tietze's Theorem. 
Lemma 32 Consider a presentation of $G$ with generators $g_{1}, \ldots, g_{k}$ and relations $R_{1}, \ldots, R_{s}$. If we add another relation $R_{s+1}$, which is valid in $G$, we get another presentation of $G$. If we express some element $g_{k+1}$ of $G$ as a product $P$ of the generators $g_{1}, \ldots, g_{k}$ we get a new presentation of $G$ with generators $g_{1}, \ldots, g_{k+1}$ and with defining relations $R_{1}, \ldots, R_{s}, g_{k+1}^{-1} P$. Conversely suppose that we can express some generator, say $g_{k}$, as a product $P$ of $g_{1}, \ldots, g_{k-1}$. Let us replace every appearence of $g_{k}$ in each relation $R_{i}, i=1, \ldots, s$ by $P$, getting a new relation $P_{i}$. Then $G$ has a presentation with generators $g_{1}, \ldots, g_{k-1}$ and with relations $P_{1}, \ldots, P_{s}$.

We start with the presentation of the mapping class group $\mathcal{M}_{g, 1}$ established in Theorem 31. The generators represent the mapping classes of corresponding homeomorphisms of the surface $S=S_{g, 1}$ represented on Figure 1. We adjoin additional generators

$$
\begin{aligned}
& b_{1}=a_{1}^{-1} r a_{1}^{-1}, \quad b_{2}=\left(t_{1} a_{1} b_{1}\right) * d_{1,2}, \quad e_{1}=\left(r d_{1,2} a_{2}^{-1}\right) * b_{2} \\
& e_{i+1}=\left(t_{i} t_{i+1}\right) * e_{i} \text { for } i=1, \ldots, g-2 .
\end{aligned}
$$

These generators also represent the corresponding twists in $\mathcal{M}_{g, 1}$. We adjoin the relations $(\mathrm{M} 1)-(\mathrm{M} 3)$. Now, by Theorem 31, generators $s, t_{i}, d_{i, j}, r$ can be expressed in $\mathcal{M}_{g, 1}$ by the formulas from Definition 4 . We substitute for each of these generators the corresponding product of $b_{2}, b_{1}, a_{1}, e_{1}, a_{2}, \ldots, a_{g-1}, e_{g-1}, a_{g}$ in all relations $(\mathrm{P} 1)-(\mathrm{P} 11)$ and in (3). We check easily that the relations (3) become trivial modulo the relations (M1) - (M3) (the last one will be proven in (17).) We want to prove that all relations (P1) - (P11) follow from relations (M1) - (M3).

Remark 5 We shall establish many auxiliary relations of increasing complexity which follow from the relations (M1) - (M3). We shall explain some standard technique which one can use (some proofs will be left to the reader). From the braid relation $a b a=b a b$ one can derive several other useful relations, like: $a * b=b^{-1} * a, a *\left(b^{2}\right)=b^{-1} *\left(a^{2}\right),(a b) * a=b$. When we want to prove that $[a, b]=0$ we shall usually try to prove that $a * b=b$. The relation $a b a=b a b$ tells us that $a$ can "jump" over $b a$ to the right becoming $b$. By consecutive jumping to the right we can prove that $a_{1}\left(e_{1} a_{1} a_{2} e_{1} e_{2} a_{2}\right)=\left(e_{1} a_{1} a_{2} e_{1} e_{2} a_{2}\right) e_{2}$. We also get $e_{1} a_{1} a_{2} e_{1} e_{2} a_{2}=e_{1} a_{2} e_{2} a_{1} e_{1} a_{2}$ and $\left(b_{1} a_{1} e_{1} a_{2}\right) * b_{2}=\left(b_{2}^{-1} a_{2}^{-1} e_{1}^{-1} a_{1}^{-1}\right) * b_{1}$. We shall say that some relations follow by $(\mathrm{J})$ - jumping, if they follow easily from (M1) by the above technique.

We start the list of the auxiliary relations.

$$
t_{i} * a_{i}=a_{i+1}, t_{i} * a_{i+1}=a_{i}, t_{i} * a_{k}=a_{k} \text { for } k \neq i, i+1,
$$


$s * a_{i}=a_{i}$ for $i=1, \ldots, g$ by $(\mathrm{J})$.

Let $w_{0}=a_{g} e_{g-1} a_{g-1} e_{g-2} \ldots e_{1} a_{1} b_{1}$.

$$
w_{0}^{-1} * b_{2}=d_{1,2}, w_{0}^{-1} * b_{1}=a_{1}, w_{0}^{-1} * a_{i}=e_{i}, w_{0}^{-1} * e_{i}=a_{i+1},
$$

$d_{1,2} * b_{1}=b_{1}^{-1} * d_{1,2}, d_{1,2} * e_{2}=e_{2}^{-1} * d_{1,2},\left[d_{1,2}, a_{i}\right]=1,\left[d_{1,2}, e_{j}\right]=1$, for $j \neq 2$, $\left[d_{1,2}, t_{j}\right]=1$ for $j \neq 2$.

Proof of (5) We have $w_{0}^{-1} * b_{2}=\left(\right.$ by (M1)) $\left(b_{1}^{-1} a_{1}^{-1} e_{1}^{-1} a_{2}^{-1}\right) * b_{2}=d_{1,2}$. Other results of conjugation by $w_{0}$ follow by $(\mathrm{J})$. Now $d_{1,2} * b_{1}=\left(b_{1}^{-1} a_{1}^{-1} e_{1}^{-1} a_{2}^{-1} b_{2} a_{2} e_{1} a_{1} b_{1}\right) * b_{1}=($ by $\mathrm{J})$ $\left(b_{1}^{-1} a_{1}^{-1} e_{1}^{-1} a_{2}^{-1} b_{1}^{-1} a_{1}^{-1} e_{1}^{-1} a_{2}^{-1}\right) * b_{2}=$ (by jumping from left side to the right) $\left(b_{1}^{-1} b_{1}^{-1} a_{1}^{-1} e_{1}^{-1} a_{2}^{-1}\right) * b_{2}=b_{1}^{-1} * d_{1,2}$.

Other relations follow from (M1) by conjugation by $w_{0}^{-1}$.

$$
\begin{gathered}
a_{k} * d_{i, j}=d_{i, j} \text { for all } i, j, k, \text { by }(\mathrm{J}) \text { and }(4) \text { and }(5) . \\
t_{i} * t_{i+1}=t_{i+1}^{-1} * t_{i} \text { for } i=1,2, \ldots, g-2,
\end{gathered}
$$

(by the calculations similar to the proof of Lemma 21 (iv)), $\left[t_{i}, t_{k}\right]=1$ for $|i-k|>1,\left[t_{i}, s\right]=1$ for $i>1$, by (M1).

Using relations (5) and (7) we can write the elements $d_{i, j}$ in a different way.

$$
d_{i, i+1}=\left(t_{i-1} t_{i} t_{i-2} t_{i-1} \ldots t_{1} t_{2}\right) * d_{1,2} \text { for } i>0,
$$

$d_{-i-1,-i}=\left(t_{i-1}^{-1} t_{i}^{-1} t_{i-2}^{-1} t_{i-1}^{-1} \ldots t_{1}^{-1} t_{2}^{-1}\right) * d_{-2,-1}$ for $i>0$,

$d_{i, i+1}=\left(t_{i-1} t_{i}\right) * d_{i-1, i}, \quad t_{k} * d_{i, i+1}=d_{i, i+1}$ for $|k-i| \neq 1$.

Let $w_{1}=a_{2} e_{1} a_{1} b_{1}^{2} a_{1} e_{1} a_{2}$.

$$
\left(b_{1} a_{1} e_{1}\right)^{4}=b_{2} w_{1} b_{2} w_{1}^{-1}, w_{1} * b_{2}=w_{1}^{-1} * b_{2},\left[w_{1} * b_{2}, b_{2}\right]=1
$$

Proof of (9) We have $b_{2} w_{1} b_{2}=\left(\right.$ by (M2)) $\left(b_{1} a_{1} e_{1} a_{2}\right)^{5}=($ as in the proof of Lemma $21(\mathrm{v}))\left(b_{1} a_{1} e_{1}\right)^{4} w_{1}=($ by $(\mathrm{J})) w_{1}\left(b_{1} a_{1} e_{1}\right)^{4}$.

Also $b_{2}\left(b_{1} a_{1} e_{1}\right)^{4}=\left(b_{1} a_{1} e_{1}\right)^{4} b_{2}$, by (M1).

Therefore $w_{1} b_{2} w_{1}^{-1}=b_{2}^{-1}\left(b_{1} a_{1} e_{1}\right)^{4}=\left(b_{1} a_{1} e_{1}\right)^{4} b_{2}^{-1}=w_{1}^{-1} b_{2} w_{1} \quad$ commutes with $b_{2}$.

(10) $s t_{1} s=b_{1} a_{1} e_{1} a_{2}^{2} e_{1} a_{1} b_{1} t_{1}=t_{1} b_{1} a_{1} e_{1} a_{2}^{2} e_{1} a_{1} b_{1}$ hence $s t_{1} s t_{1}=t_{1} s t_{1} s$ 
Proof of (10) We have a sequence of transformations by $(\mathrm{J})$.

$s t_{1} s=$

$b_{1} a_{1} a_{1} b_{1} e_{1} a_{1} a_{2} e_{1} b_{1} a_{1} a_{1} b_{1}=b_{1} a_{1} a_{1} e_{1} b_{1} a_{1} b_{1} a_{2} e_{1} a_{1} a_{1} b_{1}=$ $b_{1} a_{1} a_{1} e_{1} a_{1} b_{1} a_{2} a_{1} e_{1} a_{1} a_{1} b_{1}=b_{1} a_{1} e_{1} a_{1} e_{1} b_{1} a_{2} e_{1} e_{1} a_{1} e_{1} b_{1}=$ $b_{1} a_{1} e_{1} a_{1} b_{1} a_{2} e_{1} a_{2} e_{1} a_{1} b_{1} e_{1}=b_{1} a_{1} e_{1} a_{2} a_{1} b_{1} a_{2} e_{1} a_{1} b_{1} a_{2} e_{1}=$ $b_{1} a_{1} e_{1} a_{2} a_{2} e_{1} a_{1} b_{1} t_{1}$.

The second equality follows immediately by symmetry.

Let $w_{2}=e_{2} a_{2} e_{1} a_{1}^{2} e_{1} a_{2} e_{2}$.

$$
\left(a_{1} e_{1} a_{2}\right)^{4}=t_{1}^{2} a_{1}^{2} a_{2}^{2}=d_{1,2} d_{-2,-1}, \quad\left[d_{1,2}, d_{-2,-1}\right]=1 .
$$

$d_{-2,-1}=w_{2} * d_{1,2}=w_{2}^{-1} * d_{1,2}=\left(b_{1} a_{1} e_{1} a_{2}\right) * b_{2}$

Proof of (11) We have $d_{-2,-1}=\left(s^{-1} t_{1}^{-1} s^{-1}\right) * d_{1,2}=($ by $(10))$

$\left(\left(b_{1} a_{1} e_{1} a_{2} a_{2} e_{1} a_{1} b_{1}\right)^{-1} t_{1}^{-1} b_{1}^{-1} a_{1}^{-1} e_{1}^{-1} a_{2}^{-1}\right) * b_{2}=($ by $(\mathrm{J}))$

$\left(\left(b_{1} a_{1} e_{1} a_{2} a_{2} e_{1} a_{1} b_{1}\right)^{-1} b_{1}^{-1} a_{1}^{-1} e_{1}^{-1} a_{2}^{-1}\right) * b_{2}=($ by $(9)) \quad\left(b_{1} a_{1} e_{1} a_{2}\right) * b_{2}$.

Conjugating (9) by $\left(b_{1}^{-1} a_{1}^{-1} e_{1}^{-1} a_{2}^{-1}\right)$ we get $\left(a_{1} e_{1} a_{2}\right)^{4}=d_{1,2} d_{-2,-1}$. Also, by (M1),

$t_{1}^{2} a_{1}^{2} a_{2}^{2}=\left(a_{1} e_{1} a_{2}\right)^{4}$. This proves the first relation. The second relation follows from it by (5). Conjugating (9) by $w_{0}^{-1}$ we get, by (5), $\left(a_{1} e_{1} a_{2}\right)^{4}=$ $d_{1,2} w_{2} d_{1,2} w_{2}^{-1}$ and $w_{2} * d_{1,2}=w_{2}^{-1} * d_{1,2}$. Therefore, from the first relation, $d_{-2,-1}=w_{2} * d_{1,2}=w_{2}^{-1} * d_{1,2}=\left(b_{1} a_{1} e_{1} a_{2}\right) * b_{2}$.

Definition 6 If $A$ is a product of the generators we denote by $A^{\prime}$ the element obtained from $A$ by replacing each generator by its inverse. We call $A^{\prime}$ the element symmetric to $A$.

Remark 6 Relations (M1) and (M2) are symmetric. They remain valid when we replace each generator by its inverse. Therefore every relation between some elements of $\mathcal{M}_{g, 1}$ (products of generators) which follows from (M1) and (M2) remains valid if we replace each element by the element symmetric to it.

$$
\text { For } i+j \neq 0 \quad d_{i, j} \text { is symmetric to } d_{-j,-i}^{-1} \text {. }
$$

Proof of (12) Element $d_{1,2}$ is symmetric to $d_{-2,-1}^{-1}$, by (11). Also $t_{1}^{\prime}=t_{1}^{-1}$ and $s^{\prime}=s^{-1}$. We see immediately that $d_{i, j}^{\prime}=d_{-j,-i}^{-1}$ for $i>0$. If $i<0$ and $i+j>0$ then 
$d_{i, j}=\left(t_{-i-1}^{-1} \ldots t_{1}^{-1} s^{-1} t_{j-1} \ldots t_{2}\right) * d_{1,2}$

and $d_{-j,-i}^{-1}=\left(t_{j-1}^{-1} \ldots t_{1}^{-1} s^{-1} t_{-i} \ldots t_{2}\right) * d_{1,2}^{-1}$.

Jumping with the positive powers of $t_{k}$ to the left we get

$d_{-j,-i}^{-1}=\left(t_{-i-1} \ldots t_{1} s t_{j-1}^{-1} \ldots t_{2}^{-1} s^{-1} t_{1}^{-1} s^{-1}\right) * d_{1,2}^{-1}=$

$\left(t_{-i-1} \ldots t_{1} s t_{j-1}^{-1} \ldots t_{2}^{-1}\right) * d_{1,2}^{\prime}=d_{i, j}^{\prime}$.

$$
d_{i+1, i+2}=\left(t_{i}^{-1} t_{i+1}^{-1}\right) * d_{i, i+1}=\left(t_{i} t_{i+1}\right) * d_{i, i+1} \text { for } i=1, \ldots, g-2
$$

Proof of (13) For $i=1$ we have $\left(t_{2} t_{1}^{2} t_{2}\right) * d_{1,2}=($ by 11)

$\left(e_{2} a_{2} a_{3} e_{2} e_{1} a_{1} a_{2} e_{1} e_{1} a_{1} a_{2} e_{1} e_{2} a_{2} a_{3} e_{2} e_{2}^{-1} a_{2}^{-1} e_{1}^{-1} a_{1}^{-2} e_{1}^{-1} a_{2}^{-1} e_{2}^{-1}\right) * d_{-2,-1}$

$=(\operatorname{by}(\mathrm{J}))\left(e_{2} a_{2} a_{3} e_{2} e_{1} a_{1} a_{2} e_{1} e_{1} a_{2} e_{2} a_{3} a_{1}^{-1} e_{1}^{-1} a_{2}^{-1} e_{2}^{-1}\right) * d_{-2,-1}=($ by $(\mathrm{J}))$

$\left(e_{2} a_{2} a_{3} e_{2} e_{1} a_{1} a_{2} e_{1} e_{1} a_{1}^{-1} a_{2} e_{1}^{-1} e_{2} a_{2}^{-1} a_{3} e_{2}^{-1}\right) * d_{-2,-1}=($ by $(\mathrm{J}))$

$\left(e_{2} a_{2} a_{3} e_{2} e_{1} a_{2} e_{1}^{-1} a_{1} a_{1} e_{1} a_{2} e_{1}^{-1} e_{2} a_{2}^{-1} a_{3} e_{2}^{-1}\right) * d_{-2,-1}=($ by $(\mathrm{J}))$

$\left(a_{3}^{-1} e_{2} a_{2} e_{1} a_{1}^{2} e_{1} a_{2} e_{2} a_{3}\right) * d_{-2,-1}=\left(\right.$ by (6) and (11)) $a_{3}^{-1} * d_{1,2}=\left(\right.$ by (6)) $d_{1,2}$.

So (13) is true for $i=1$. We continue by induction. Conjugating relation (13) by $t_{i} t_{i+1} t_{i+2}$ we get, by (8) and (7), relation (13) for index $i+1$.

$$
t_{i}^{2}=d_{i, i+1} d_{-i-1,-i} a_{i}^{-2} a_{i+1}^{-2}
$$

Proof of (14) The relation is true for $i=1$, by (11). We proceed by induction.

$t_{i+1}^{2}=\left(t_{i}^{-1} t_{i+1}^{-1}\right) * t_{i}^{2}=\left(t_{i}^{-1} t_{i+1}^{-1}\right) *\left(d_{i, i+1} d_{-i-1,-i} a_{i}^{-2} a_{i+1}^{-2}\right)$

$=\left(\right.$ by $(7),(13),(8)$ and (4)) $d_{i+1, i+2} d_{-i-2,-i-1} a_{i+1}^{-2} a_{i+2}^{-2}$.

$$
d_{-i, i} \text { is symmetric to } d_{-i, i}^{-1} \text {. }
$$

Proof of (15) The relation is true for $i=1$. The general case follows from (14), (4), (5), (12) and the definitions.

$$
\left[b_{1}, d_{-2,2}\right]=1
$$

Proof of (16) By the definition $d_{-2,2}=\left(t_{1}^{-1} d_{1,2}\right) *\left(s^{2} a_{1}^{4}\right)=($ by $(4))$ $a_{2}^{4}\left(\left(d_{1,2} t_{1}^{-1}\right) * s^{2}\right)$.

Now $t_{1}^{-1} * s=t_{1}^{-1} s t_{1} s s^{-1}=($ by $(10)) b_{1} a_{1} e_{1} a_{2}^{2} e_{1} a_{1}^{-1} b_{1}^{-1}$. 
Taking squares we get $t_{1}^{-1} * s^{2}=b_{1} a_{1} e_{1} a_{2}^{2} e_{1}^{2} a_{2}^{2} e_{1} a_{1}^{-1} b_{1}^{-1}$ and $d_{-2,2}=a_{2}^{4} d_{1,2} b_{1} a_{1} e_{1} a_{2}^{2} e_{1}^{2} a_{2}^{2} e_{1} a_{1}^{-1} b_{1}^{-1} d_{1,2}^{-1}$. Now $b_{1}$ commutes with $d_{-2,2}$, by (4), (5) and (J).

$$
\left(t_{i} t_{i+1}\right) * e_{i}=e_{i+1} \text { for } i=1, \ldots, g-2 .
$$

Proof of (17) We have, by $(\mathrm{J}),\left(t_{i} t_{i+1}\right) * e_{i}=$ $\left(e_{i} a_{i} a_{i+1} e_{i} e_{i+1} a_{i+1} a_{i+2} e_{i+1}\right) * e_{i}=\left(e_{i} a_{i} a_{i+1} e_{i+1} e_{i} a_{i+1}\right) * e_{i}=e_{i+1}$.

$$
\left[b_{1}, d_{i, i+1}\right]=1 \text { if } i>1
$$

$\left[e_{k}, d_{i, i+1}\right]=1$ if $|k-i| \neq 1, i>0, \quad d_{i, i+1} * e_{k}=e_{k}^{-1} * d_{i, i+1} \quad$ if $|k-i|=1$.

Proof of (18) By (13) and (J)

$d_{2,3}=\left(e_{1}^{-1} a_{2}^{-1} e_{2}^{-1} a_{3}^{-1} a_{1}^{-1} e_{1}^{-1} a_{2}^{-1} e_{2}^{-1} b_{1}^{-1} a_{1}^{-1} e_{1}^{-1} a_{2}^{-1}\right) * b_{2}=$ $\left(e_{1}^{-1} a_{2}^{-1} e_{2}^{-1} a_{3}^{-1} a_{1}^{-1} b_{1}^{-1} e_{1}^{-1} a_{1}^{-1} a_{2}^{-1} e_{1}^{-1} e_{2}^{-1} a_{2}^{-1}\right) * b_{2}$.

Now $b_{1}$ commutes with $d_{2,3}$, by $(\mathrm{J})$. For $i>2$ we have $b_{1}$ commutes with $d_{i, i+1}$ by (M1) and (8). Conjugating by $a_{1} b_{1} t_{1}$ we get, by (7) and (J), $\left[e_{1}, d_{i, i+1}\right]=1$, for $i>2$. We also have $d_{1,2} * b_{1}=b_{1}^{-1} * d_{1,2}$, by (5). We conjugate this equality by $u=a_{1} b_{1} t_{1} t_{2}$ and get $d_{2,3} * e_{1}=e_{1}^{-1} * d_{2,3}$, by (8) and the first part of the proof. Conjugating relations (5) and the above relations by suitable products $t_{i} t_{i+1}$ we get all remaining relations, by (17).

$$
\left[b_{1}, d_{1,2} s d_{1,2}\right]=1, \text { hence }\left[s, d_{1,2} s d_{1,2}\right]=1,
$$

$\left[e_{j}, d_{i, i+1} t_{j} d_{i, i+1}\right]=1$, hence $\left[t_{j}, d_{i, i+1} t_{j} d_{i, i+1}\right]=1$, if $|i-j|=1$.

Proof of (19) By (5) we have $\left(d_{1,2} s d_{1,2}\right) * b_{1}=\left(d_{1,2} b_{1} a_{1} a_{1} b_{1} b_{1}^{-1}\right) * d_{1,2}=$ $\left(d_{1,2} b_{1}\right) * d_{1,2}=b_{1}$. The other case is similar, but we use (18) instead of (5).

Remark 7 Relation $u v u v=v u v u$ implies $(u v) * u=v^{-1} * u$ and $\left(u^{-1} v^{-1}\right) *$ $u=v * u$. Relations (19) will be often used in this form.

Observe that relations (4) - (14) imply in particular that relations (P1) and relations $(\mathrm{P} 3)-(\mathrm{P} 7)$ follow from $(\mathrm{M} 1)$ and $(\mathrm{M} 2)$. We shall prove now that relations (P8) follow from (M1) and (M2). 
Definition 7 We say that homeomorphism $t_{k}$ (respectively $t_{k}^{-1} d_{k+1, k+2}$ or $s$ ) moves a curve $\delta_{i, j}$ properly if it takes it to some curve $\delta_{p, q}$.

Lemma 33 If $t_{k}$ (respectively $s$ ) moves curve $\delta_{i, j}$ to some curve $\delta_{p, q}$ then $t_{k} * d_{i, j}=d_{p, q}$ (respectively $s * d_{i, j}=d_{p, q}$ ).

Remark 8 Since the action of $t_{k}$ and $s$ on a curve $\delta_{i, j}$ is described by Lemma 24 and is easily determined, Lemma 33 helps us to understand the result of the conjugation. In fact the action corresponds exactly to relations (P8), so we have to prove that relations (P8) follow from (M1) and (M2).

Proof of Lemma 33 We know that $\left[t_{i}, d_{i, i+1}\right]=1$, by (8), (7), and (5).

If $i<0$ and $i+j=1$ then $t_{j-1}^{-1} * d_{i, j}=\left(t_{j-1}^{-1} t_{j-2}^{-1} \ldots t_{1}^{-1} s^{-1} t_{j-1} \ldots t_{2}\right) * d_{1,2}=$ $d_{i-1, j-1}$.

For $i>0$ or $i<0, i+j>0$ all other cases of conjugation by $t_{k}$ follow from (5), (7) and the definitions. The other cases of $i \neq-j$ follow by symmetry.

Consider conjugation by $s$ for $i \neq-j$. Again it suffices to consider $i>0$ or $i<0, i+j>0$. The other cases follow by symmetry. We have $s^{-1} * d_{1, j}=d_{-1, j}$. If $i>1$ then $d_{i, j}=($ by $(7)) \quad\left(t_{i-1} \ldots t_{2} t_{j-1} \ldots t_{3}\right) * d_{2,3}$ and $s * d_{i, j}=d_{i, j}$, by (7), (6) and (18).

If $i<1$ then $d_{i, j}=($ by 7$) \quad\left(t_{-i-1}^{-1} \ldots t_{2}^{-1} t_{j-1} \ldots t_{3}\right) * d_{-2,3}$. Also $s^{-1} * d_{-2,3}=$ $\left(s^{-1} t_{1}^{-1} s^{-1} t_{2}\right) * d_{1,2}=\left(s^{-1} t_{1}^{-1} s^{-1} t_{1}^{-1}\right) * d_{2,3}=($ by $(10))\left(t_{1}^{-1} s^{-1} t_{1}^{-1} s^{-1}\right) * d_{2,3}=$ $d_{-2,3}$ (by (6) and (18)). So $s^{-1} * d_{i, j}=d_{i, j}$, by $(7)$.

We now consider conjugation of $d_{-j, j}$. Clearly $s * d_{-1,1}=d_{-1,1}$, by (M1). Also $s * d_{-2,2}=d_{-2,2}$, by (6) and (16). For $i>1$ we have $\left[s, t_{i}\right]=1$, by (M1), hence $s * d_{-j, j}=d_{-j, j}$ for all $j$, by the first part of the proof.

Consider conjugation by $t_{k}$.

For $k>j$ we have $t_{k} * d_{-j, j}=d_{-j, j}$, by (7) and the first part.

Curves $t_{j}\left(\delta_{-j, j}\right)$ and $t_{j-1}\left(\delta_{-j, j}\right)$ are not of the form $\delta_{p, q}$.

Consider $k=j-2$ (the other cases follow by conjugation and by the first part of the proof). We have

$d_{-j, j}=\left(d_{j-1, j} t_{j-1}^{-1} t_{j-2}^{-1} d_{j-2, j-1}\right) * d_{2-j, j-2}=($ by (8) and (13))

$\left(t_{j-2}^{-1} t_{j-1}^{-1} d_{j-2, j-1} t_{j-1} t_{j-2} t_{j-1}^{-1} t_{j-2}^{-1} d_{j-2, j-1}\right) * d_{2-j, j-2}=($ by (7) and (8))

$\left(t_{j-2}^{-1} t_{j-1}^{-1} t_{j-2}^{-1} d_{j-2, j-1} t_{j-1} d_{j-2, j-1}\right) * d_{2-j, j-2}$. Now, by (7) and (19),

$t_{j-2}^{-1} * d_{-j, j}=\left(t_{j-2}^{-1} t_{j-1}^{-1} t_{j-2}^{-1} d_{j-2, j-1} t_{j-1} d_{j-2, j-1} t_{j-1}^{-1}\right) * d_{2-j, j-2}=d_{-j, j}$ (by the previous case). 
We now pass to the biggest task of this section: the relations (P2).

$$
d_{i, j} \text { commutes with } d_{-1,1} \text { if } i, j \neq \pm 1 \text {, }
$$

$d_{i, j}$ commutes with $d_{k, k+1}$ if all indices are distinct.

Proof of (20) We know by Lemma 33 that $d_{i, j}$ commutes with $s$, hence also with $d_{-1,1}$. Consider the other cases. We assume first that $k>0$. Consider curves $\delta_{i, j}$ and $\delta_{k, k+1}$. We want to move the curves properly to some standard position by application of products of $s$ and $t_{m}$ 's. This moves holes (see definition 5) and corresponds to conjugation of $d_{i, j}$ and $d_{k, k+1}$, by the previous lemma.

Case $1 \quad i \neq-j$ Observe that for every $k$ either $t_{k+1} t_{k}$ or $t_{k+1}^{-1} t_{k}^{-1}$ moves $\delta_{i, j}$ properly. Both products take $\delta_{k+1, k+2}$ onto $\delta_{k, k+1}$ and conjugation of $d_{k+1, k+2}$ by either product produces $d_{k, k+1}$. If either $|i|$ or $|j|$ is bigger than $k+1$ we may assume, moving $\delta_{i, j}$ properly, and not moving $\delta_{k, k+1}$, that either $|i|$ or $|j|$ is equal to $k+2$. Then either $t_{k} t_{k+1}$ or $t_{k}^{-1} t_{k+1}^{-1}$ moves $\delta_{i, j}$ properly and moves $\delta_{k, k+1}$ to $\delta_{k+1, k+2}$ and leaves at most one index $|i|$ or $|j|$ bigger than $k+1$. Applying this procedure again, if necessary, we may assume $j<k$. If $j>0$ we can move $\delta_{i, j}$ properly, without moving $\delta_{k, k+1}$, and get a curve $\delta_{i, j}$ with $j<0$. Now applying consecutive products $t_{m+1} t_{m}$ or $t_{m+1}^{-1} t_{m}^{-1}$ we reach $k=1$. Further moves will produce one of the following three cases:

Case 1a $d_{1,2}$ commutes with $d_{-2,-1}$. True, by (11).

Case 1b $d_{1,2}$ commutes with $d_{-3,-1}$ or $d_{-3,-2}$. We can conjugate $d_{-3,-2}$ by $t_{1}$ and get $d_{-3,-1}$. Now $d_{-3,-1}=t_{2}^{-1} * d_{-2,-1}=($ by $(11))\left(e_{2}^{-1} a_{3}^{-1} a_{2}^{-1} e_{2}^{-1} w_{2}\right) *$ $d_{1,2}=($ by $(\mathrm{J})$ and $(5))\left(e_{2}^{-1} a_{3}^{-1} e_{1} a_{1} d_{1,2}^{-1} e_{2}^{-1} a_{2}^{-1} e_{1}^{-1}\right) * a_{1}=($ by $(\mathrm{J})$ and $(5))$ $\left(e_{2}^{-1} d_{1,2}^{-1} a_{3}^{-1} e_{2}^{-1} e_{1} a_{1} a_{2}^{-1} e_{1}^{-1}\right) * a_{1}$.

The last expression commutes with $d_{1,2}$, by $(\mathrm{J})$ and (5).

Case 1c $d_{-2,-1}$ commutes with $d_{3,4}$. The proof is rather long. We consider relation (M3): $d_{3}=a_{3}^{-1} a_{2}^{-1} a_{1}^{-1} d_{1,2} d_{1,3} d_{2,3}$, where

$d_{3}=\left(b_{1}^{-1} b_{2} a_{2} e_{1} e_{2} a_{2} a_{3} e_{2} b_{1}^{-1} a_{1}^{-1} e_{1}^{-1} a_{2}^{-1}\right) * b_{2}=$

$\left(b_{1}^{-1} b_{2} a_{2} e_{1} e_{2} a_{2} a_{3} e_{2} b_{2} a_{2} e_{1} a_{1}\right) * b_{1}$. It follows, by $(\mathrm{J})$, that $d_{3}$ commutes with $a_{2} e_{1} e_{2} a_{2} a_{3} e_{2}$, hence $d_{3}=\left(b_{1}^{-1}\left(\left(a_{2} e_{1} e_{2} a_{2} a_{3} e_{2}\right)^{-1} * b_{2}\right) b_{1}^{-1} a_{1}^{-1} e_{1}^{-1} a_{2}^{-1}\right) * b_{2}$. We now conjugate relation (M3) by $u=\left(a_{4} e_{3} a_{3} e_{2} a_{2} e_{1} a_{1} b_{1}\right)^{-1} a_{3} e_{2} a_{2} e_{1} a_{1} b_{1}$. When we write $d_{1,2}=\left(b_{1}^{-1} a_{1}^{-1} e_{1}^{-1} a_{2}^{-1}\right) * b_{2}$ we see that $d_{1,2}$ commutes with $u$, by $(\mathrm{J})$. 
All other factors on the RHS commute with $u$ by $(\mathrm{J})$, so we may replace $d_{3}$ in the relation (M3) by $u * d_{3}=$ $\left(a_{4} e_{3} a_{3} e_{2} a_{2} e_{1} a_{1} b_{1}\right)^{-1} *\left(\left(a_{3} e_{2} a_{2} e_{1} a_{1}\left(\left(a_{2} e_{1} e_{2} a_{2} a_{3} e_{2}\right)^{-1} * b_{2}\right) b_{1}^{-1} a_{1}^{-1} e_{1}^{-1} a_{2}^{-1}\right) * b_{2}\right)$.

We conjugate each term by $\left(a_{4} e_{3} a_{3} e_{2} a_{2} e_{1} a_{1} b_{1}\right)^{-1}$ and get $u * d_{3}=\left(e_{3} a_{3} e_{2} a_{2} e_{1}\left(\left(e_{2} a_{2} a_{3} e_{2} e_{3} a_{3}\right)^{-1} * d_{1,2}\right) a_{1}^{-1} e_{1}^{-1} a_{2}^{-1} e_{2}^{-1}\right) * d_{1,2}$.

We now conjugate each term of relation (M3) by $t_{2}^{-1} t_{3}^{-1}=$ $e_{2}^{-1} a_{3}^{-1} e_{3}^{-1} a_{4}^{-1} a_{2}^{-1} e_{2}^{-1} a_{3}^{-1} e_{3}^{-1}$.

The RHS becomes $a_{4}^{-1} a_{3}^{-1} a_{1}^{-1}\left(t_{2}^{-1} * d_{1,2}\right)\left(\left(t_{2}^{-1} t_{3}^{-1} t_{2}\right) * d_{1,2}\right) d_{3,4}$.

The LHS becomes

$\left(e_{2}^{-1} a_{3}^{-1} e_{3}^{-1} a_{4}^{-1}\right) *\left(\left(e_{1}\left(\left(e_{2} a_{2} a_{3} e_{2} e_{3} a_{3}\right)^{-1} * d_{1,2}\right)\left(a_{1}^{-1} e_{1}^{-1} a_{2}^{-1} e_{2}^{-1}\right)\right) * d_{1,2}\right)$.

We conjugate each bracket.

$\left(e_{2}^{-1} a_{3}^{-1} e_{3}^{-1} a_{4}^{-1}\right) * e_{1}=e_{1}$.

$\left(e_{2}^{-1} a_{3}^{-1} e_{3}^{-1} a_{4}^{-1}\left(e_{2} a_{2} a_{3} e_{2} e_{3} a_{3}\right)^{-1}\right) * d_{1,2}=($ by $(\mathrm{J})$ and $(5))\left(t_{3}^{-1} t_{2}^{-1}\right) * d_{1,2}$.

$\left(e_{2}^{-1} a_{3}^{-1} e_{3}^{-1} a_{4}^{-1} a_{1}^{-1} e_{1}^{-1} a_{2}^{-1} e_{2}^{-1}\right) * d_{1,2}=($ by $(\mathrm{J})$ and $(5)) \quad\left(a_{1}^{-1} e_{1}^{-1} t_{2}^{-1}\right) * d_{1,2}$.

We shall prove that all terms of the obtained equation commute with $d_{-2,-1}$, except possibly for $d_{3,4}$. Therefore $d_{3,4}$ also commute with $d_{-2,-1}$. Clearly $a_{1}, e_{1}, a_{2}, a_{3}, a_{4}, t_{1}, t_{3}$ commute with $d_{-2,-1}$ by (5) and symmetry. Also $d_{1,2}$ commutes with $d_{-2,-1}$ by Case 1 a and $d_{1,3}$ and $d_{2,3}$ commute with $d_{-2,-1}$ by Case $1 \mathrm{~b}$. Therefore $t_{2}^{-1} * d_{1,2}=t_{1} * d_{2,3}$ commute with $d_{-2,-1}$ and $\left(t_{2}^{-1} t_{3}^{-1} t_{2}\right) *$ $d_{1,2}=($ by $(7)$ and $(5)) \quad\left(t_{3} t_{2}^{-1}\right) * d_{1,2}$ commutes with $d_{-2,-1}$. It follows that $d_{3,4}$ commutes with $d_{-2,-1}$

Case $2 i=-j$ We have to prove that $d_{k, k+1}$ commutes with $d_{-j, j}$ if $j \neq$ $k, k+1$. If $j<k$ then the result follows by Lemma 33 and by Case 1. If $j>k+2$ we can properly move $\delta_{k, k+1}$ to $\delta_{j-2, j-1}$, without moving $\delta_{-j, j}$. So we may assume $j=k+2$. We have

$d_{-j, j}=\left(d_{k+1, k+2} t_{k+1}^{-1} t_{k}^{-1} d_{k, k+1}\right) * d_{-k, k}=($ by $(6)$ and (14))

$\left(d_{-k-2,-k-1}^{-1} t_{k+1} t_{k} d_{-k-1,-k}^{-1}\right) * d_{-k, k}$.

We conjugate $d_{k, k+1}$ by $\left(d_{-k-2,-k-1}^{-1} t_{k+1} t_{k} d_{-k-1,-k}^{-1}\right)^{-1}$ and get, by Case 1 and (13), $\left(d_{-1-k,-k} t_{k}^{-1} t_{k+1}^{-1} d_{-k-2,-k-1}\right) * d_{k, k+1}=d_{-1-k,-k} * d_{k+1, k+2}=d_{k+1, k+2}$, which commutes with $d_{-k, k}$ by the first part of Case 2 .

Suppose now that $k<0$. Cases $1 \mathrm{a}$ and $1 \mathrm{~b}$ follow by symmetry. In the Case $1 \mathrm{c}$ we arrive by symmetry at the situation where we have to prove that $d_{1,2}$ commutes with $d_{-4,-3}$. Conjugating by $t_{2} t_{1} t_{3} t_{2}$ we get a pair $d_{3,4}, d_{-2,-1}$, which commutes by Case 1c. Now Case 2 follows by symmetry. 
Lemma 34 If $t_{k}^{-1} d_{k, k+1}$ takes a curve $\delta_{i, j}$ to $\delta_{p, q}$ then $t_{k}^{-1} d_{k, k+1} * d_{i, j}=d_{p, q}$.

Proof We shall list the relevant cases. If $i$ or $j$ is equal to $k$ it becomes $k+1$, if $i$ or $j$ is equal to $-k$ it becomes $-k-1$. In particular $t_{k}^{-1} d_{k, k+1}\left(\delta_{-k, k}\right)=$ $\delta_{-k-1, k+1}$. Indices $k+1$ and $-k-1$ are forbidden, they do not move properly, except for $\delta_{k, k+1}$ and $\delta_{-k-1,-k}$ which are fixed by $t_{k}^{-1} d_{k, k+1}$. Other indices $i$, $j$ do not change.

We now pass to the proof of the Lemma. If $i=-j$ the result follows from the definitions and from (20) and Lemma 33. Suppose $i \neq-j$. If $i$ and $j$ are different from $k$ then $d_{i, j}$ commutes with $d_{k, k+1}$, by (20), and $t_{k}^{-1}$ moves $\delta_{i, j}$ properly, so we are done by Lemma 33. If $i$ and $j$ are different from $-k$ we can replace $t_{k}^{-1} d_{k, k+1}$ by $t_{k} d_{-k-1,-k}^{-1} a_{k}^{2} a_{k+1}^{2}$, using (14), and we are done by a similar argument.

Lemmas 33 and 34 allow us to reduce relations (P2) to relatively small number of cases. We can apply product of half-twists $h_{k, k+1}$ and $h_{-k-1,-k}$ either in the same direction, conjugating by $t_{k}$, or in opposite directions, conjugating by $t_{k}^{-1} d_{k, k+1}$, and move properly curves corresponding to elements $d_{p, q}$ in the relations (P2) into a small number of standard configurations.

$$
d_{i, j} \text { commutes with } d_{r, s} \text { if } r<s<i<j \text { or } i<r<s<j .
$$

Proof of (21) Moving curves $\delta_{i, j}$ and $\delta_{r, s}$ properly we can arrive at a situation $s=r+1$ or $j=i+1$ or $-r=s=1$ or $-i=j=1$. In particular if $i<r<s<j$ and $r=-s$ then conjugating by $t_{2} d_{2,3}^{-1} \ldots t_{s-1} d_{1-s, s-1}^{-1}$ we get a pair $d_{-1,1}, d_{i, j}$. Then (21) follows from (20).

$$
d_{r, i}^{-1} * d_{i, j}=d_{r, j} * d_{i, j} \text { if } r<i<j
$$

Proof of (22) Indices $(i,-i)$ move together (either remain fixed or move to $(i-1,1-i)$ or to $(i+1,-i-1))$ when we conjugate by $t_{k}$ or $s$ or $t_{k}^{-1} d_{k, k+1}$. Moving curves properly we can arrive at one of the following four cases depending on the pairs of opposite indices.

Case 1 There is no pair of opposite numbers among $r, i, j$. We may assume that $(r, i, j)=(1,2,3)$. 
$d_{1,2}^{-1} * d_{2,3}=\left(\right.$ by (13)) $\left(d_{1,2}^{-1} t_{1}^{-1} t_{2}^{-1}\right) * d_{1,2}=\left(\right.$ by (5) and (19)) $\quad\left(t_{1}^{-1} t_{2}\right) * d_{1,2}$.

$d_{1,3} * d_{2,3}=\left(t_{2} d_{1,2} t_{2}^{-1} t_{1} t_{2}\right) * d_{1,2}=($ by $(5)$ and $(7))\left(t_{2} t_{1} d_{1,2} t_{2}\right) * d_{1,2}=($ by (19)) $\left(t_{2} t_{1} t_{2}^{-1}\right) * d_{1,2}=($ by $(5)$ and $(7))\left(t_{1}^{-1} t_{2}\right) * d_{1,2}$.

Case $2 i=-r$ We may assume that $(r, i, j)=(-1,1,2)$.

$d_{-1,1}^{-1} * d_{1,2}=\left(s^{-2} a_{1}^{-4}\right) * d_{1,2}=($ by $(19)) \quad\left(s^{-1} d_{1,2} s\right) * d_{1,2}=d_{-1,2} * d_{1,2}$.

Case $3 r=-j$ We may assume that $(r, i, j)=(-2,1,2)$. We have to prove that $d_{-2,1}^{-1} * d_{1,2}=d_{-2,2} * d_{1,2}$. We conjugte by $d_{1,2}^{-1} t_{1}$. The right hand side becomes $s^{2} * d_{1,2}$ and the left hand side becomes $\left(d_{1,2}^{-1} t_{1} t_{1}^{-1} s^{-1} d_{1,2}^{-1} s t_{1}\right) * d_{1,2}=$ (by (5) and (19)) $\left(s d_{1,2}^{-1} s^{-1} d_{1,2}^{-1}\right) * d_{1,2}=\left(\right.$ by (19)) $s^{2} * d_{1,2}$.

Case $4 i=-j$ We may assume that $(r, i, j)=(-2,-1,1)$.

$d_{-2,-1}^{-1} * d_{-1,1}=a_{1}^{4}\left(d_{-2,-1}^{-1} * s^{2}\right)=($ by $(19)$ and symmetry $) a_{1}^{4}\left(\left(s d_{-2,-1} s^{-1}\right) *\right.$ $\left.s^{2}\right)=d_{-2,1} * d_{-1,1}$.

$$
d_{r, j}^{-1} * d_{r, i}=d_{i, j} * d_{r, i} \text { if } r<i<j .
$$

Proof of (23) Let us apply symmetry to relation (22). We get $d_{-i,-r} * d_{-j,-i}^{-1}=d_{-j,-r}^{-1} * d_{-j,-i}^{-1}$ if $-j<-i<-r$. This is relation (23) after a suitable change of indices.

$$
\left[d_{i, j}, d_{r, j}^{-1} * d_{r, s}\right]=1 \text { if } r<i<s<j
$$

Proof of (24) Again we have to consider different cases depending on pairs of opposite indices. For each of them we move curves properly to some standard position. If we apply symmetry we get $\left[d_{-j,-i}, d_{-j,-r} * d_{-s,-r}\right]=1$. Now conjugate by $d_{-j,-r}^{-1}$ and get $\left[d_{-s,-r}, d_{-j,-r}^{-1} * d_{-j,-i}\right]=1$ if $-j<-s<-i<-r$. This is again relation (24) with different pairs of opposite indices. So $i=-j$ is equivalent to $r=-s$ and $s=-j$ is equivalent to $r=-i$. We are left with the following five cases.

Case 1 There is no pair of opposite numbers among $r, i, s, j$. We may assume that $(r, i, s, j)=(1,2,3,4)$. Conjugate by $t_{3}^{-1}$.

$t_{3}^{-1} * d_{2,4}=d_{2,3}$.

$\left(t_{3}^{-1} d_{1,4}^{-1}\right) * d_{1,3}=\left(t_{2} d_{1,2}^{-1} t_{2}^{-1} t_{3}^{-1} t_{2}\right) * d_{1,2}=($ by $(7))\left(t_{2} d_{1,2}^{-1} t_{3} t_{2}^{-1} t_{3}^{-1}\right) * d_{1,2}=($ by 
(5)) $\left(t_{2} t_{3} d_{1,2}^{-1} t_{2}^{-1}\right) * d_{1,2}=($ by $(19))\left(t_{2} t_{3} t_{2}\right) * d_{1,2}=d_{1,4}$, and it commutes with $d_{2,3}$, by $(21)$.

Case $2 \quad r=-i$ We may assume that $(r, i, s, j)=(-1,1,2,3)$. We conjugate by $t_{2}^{-1}$.

$t_{2}^{-1} * d_{1,3}=d_{1,2}$.

$\left(t_{2}^{-1} d_{-1,3}^{-1}\right) * d_{-1,2}=\left(t_{2}^{-1} s^{-1} t_{2} d_{1,2}^{-1} t_{2}^{-1}\right) * d_{1,2}=($ by $(7)$ and $(19))\left(s^{-1} t_{2}\right) * d_{1,2}=$ $d_{-1,3}$, and it commutes with $d_{1,2}$, by (21).

Case $3 r=-s$ We may assume that $(r, i, s, j)=(-2,1,2,3)$. By (23) we have $d_{-2,3}^{-1} * d_{-2,2}=d_{2,3} * d_{-2,2}$, so we have to prove that $d_{1,3}$ commutes with $d_{2,3} * d_{-2,2}$. We conjugate by $t_{2}^{-1}$ and get $t_{2}^{-1} * d_{1,3}=d_{1,2}$.

$\left(t_{2}^{-1} d_{2,3}\right) * d_{-2,2}=d_{-3,3}$, and it commutes with $d_{1,2}$, by $(21)$.

Case $4 \quad r=-j$ and $i \neq-s$ We may assume that $(r, i, s, j)=(-3,1,2,3)$. By (23) we have $d_{-3,3}^{-1} * d_{-3,2}=d_{2,3} * d_{-3,2}$. After conjugation by $t_{1}^{-1} d_{2,3}^{-1}$ we have to prove that $t_{1}^{-1} * d_{-3,2}=d_{-3,1}$ commutes with $\left(t_{1}^{-1} d_{2,3}^{-1}\right) * d_{1,3}=$ $\left(t_{1}^{-1} d_{2,3}^{-1} t_{1}^{-1}\right) * d_{2,3}=($ by $(19)) d_{2,3}$. This is true by $(21)$.

Case $5 i=-s$ We may assume that $(r, i, s, j)=(-2,-1,1, m)$, where $m=2$ or $m=3$. By $(23)$ we have $d_{-2, m}^{-1} * d_{-2,1}=d_{1, m} * d_{-2,1}$. After conjugation by $s^{-1} d_{1, m}^{-1}$ we have to consider $s^{-1} * d_{-2,1}=d_{-2,-1}$ and $\left(s^{-1} d_{1, m}^{-1}\right) * d_{-1, m}$. For $m=2$ the last expression is equal $\left(s^{-1} d_{1,2}^{-1} s^{-1}\right) * d_{1,2}=d_{1,2}$, by (19). For $m=3$ we get $\left(s^{-1} d_{1,3}^{-1} s^{-1}\right) * d_{1,3}=\left(s^{-1} t_{2} d_{1,2}^{-1} t_{2}^{-1} t_{2} s^{-1}\right) * d_{1,2}=($ by (19) $) d_{1,3}$. Both elements commute with $d_{-2,-1}$, by $(21)$.

This concludes the proof of the fact that relations (P2) follow from (M1) (M3).

We now pass to the relations $(\mathrm{P} 9)-(\mathrm{P} 11)$.

Consider first relations (P9). Clearly $a_{1}$ commutes with all the elements in (P9), by (4) and (6), so it suffices to prove that $b_{1}$ commutes with these elements. It commutes with $a_{1}^{2} s, t_{1} s t_{1}, a_{2}$, and $t_{i}$, for $i>1$, by $(\mathrm{J})$. Also $b_{1}$ commutes with $d_{-2,2}$, by (16), and commutes with $d_{2,3}$ by (18). Finally it commutes with $d_{1,2} s d_{1,2}$, by (19), hence also commutes with $d_{-1,1} d_{-1,2} d_{1,2} a_{1}^{-2} a_{2}^{-1}=$ $a_{1}^{4} s^{2} s^{-1} d_{1,2} s d_{1,2} a_{1}^{-2} a_{2}^{-1}=a_{1}^{2} s d_{1,2} s d_{1,2} a_{2}^{-1}$. This proves relations (P9). Relation (P10) follows from the definitions and (M1).

We now pass to relations (P11). 
For $i=1,2,3,4$ we have $g_{i}=\left(k_{i} r\right)^{3}$. By (6) $k_{i} * a_{1}=a_{1}$ therefore it suffices to prove that $k_{i} * b_{1}=b_{1}^{-1} * k_{i}$. Then

$g_{i}=\left(k_{i} a_{1} b_{1} a_{1}\right)^{3}=\left(\right.$ by (6)) $k_{i} a_{1} b_{1} a_{1}^{2} k_{i} b_{1} k_{i} a_{1}^{2} b_{1} a_{1}=k_{i} a_{1} s k_{i} s a_{1}$ and this is exactly relation (P11) for $i=1,2,3,4$.

$$
k_{1} * b_{1}=b_{1}^{-1} * k_{1}
$$

Proof of $(25)$ Since $k_{1}=a_{1}$ the result follows from (M1).

$$
k_{2} * b_{1}=b_{1}^{-1} * k_{2}
$$

Proof of (26) Since $k_{2}=d_{1,2}$ the result follows from (5).

$$
k_{3} * b_{1}=b_{1}^{-1} * k_{3}
$$

Proof of (27) We have

$k_{3}=a_{1}^{-1} a_{2}^{-2} d_{1,2} d_{-2,1} d_{-2,2}=a_{1}^{-1} a_{2}^{-2} d_{1,2} t_{1}^{-1} s^{-1} d_{1,2} s t_{1} t_{1}^{-1} d_{1,2} s^{2} a_{1}^{4} d_{1,2}^{-1} t_{1}=$ (by (5) and (J)) $a_{1}^{-1} t_{1}^{-1} d_{1,2} s^{-1} d_{1,2} s d_{1,2} s^{2} a_{1}^{2} d_{1,2}^{-1} t_{1}=$ (by (19)) $a_{1}^{-1} t_{1}^{-1} d_{1,2} s d_{1,2} s a_{1}^{2} t_{1}=$ (by (5) and (J)) $a_{1}^{-1} t_{1}^{-1}\left(d_{1,2} b_{1} a_{1}\right)^{4} t_{1}$.

Let $u=t_{1} b_{1} d_{1,2} a_{1} b_{1}$. It follows from (5) and (J) that $u * a_{1}=d_{1,2}, u * e_{1}=b_{1}$, $u * a_{2}=a_{1}, u * d_{1,2}=a_{2}$. Conjugating (11) by $u$ we get $\left(d_{1,2} b_{1} a_{1}\right)^{4}=$ $a_{2}\left(u * d_{-2,-1}\right)$, hence $k_{3}=($ by $(4))\left(t_{1}^{-1} u\right) * d_{-2,-1}=\left(b_{1} d_{1,2} a_{1} b_{1}\right) * d_{-2,-1}$. We want to prove that $b_{1}$ and $k_{3}$ are braided. It suffices to prove it for their common conjugates. We conjugate by $b_{1}^{-1}$ and get $b_{1}$ and $\left(d_{1,2} a_{1} b_{1}\right) * d_{-2,-1}$. Now we conjugate by $a_{1}^{-1} b_{1}^{-1} d_{1,2}^{-1}$ and get, by (5), (6) and symmetry, $d_{1,2}$ and $b_{1} * d_{-2,-1}=d_{-2,-1}^{-1} * b_{1}$. Now we conjugate by $d_{-2,-1}$ and get, by $(20), d_{1,2}$ and $b_{1}$, which are braided.

$$
k_{4} * b_{1}=b_{1}^{-1} * k_{4}
$$

Proof of (28) By the definition and by (M3) we have $k_{4}=d_{3}=\left(b_{2} a_{2} e_{1} b_{1}^{-1}\right) *$ $d_{1,3}$. Conjugating $k_{4}$ and $b_{1}$ by $t_{2}^{-1} b_{1} e_{1}^{-1} a_{2}^{-1} b_{2}^{-1}$ we get $d_{1,2}$ and $b_{1}$, which are braided, by (5).

$$
g_{5}=s a_{1}^{2} k_{5} s a_{1}^{2} k_{5}^{-1}
$$


Proof of (29) By the definition $g_{5}=\left(r k_{5} r k_{5}^{-1}\right)^{2}$ where $k_{5}=a_{2} d_{1,2}^{-1} t_{1}$. We shall prove that $r$ commutes with $k_{5} r k_{5}^{-1}$. Then $g_{5}=r^{2} k_{5} r^{2} k_{5}^{-1}=s a_{1}^{2} k_{5} s a_{1}^{2} k_{5}^{-1}$, as required.

$k_{5} r k_{5}^{-1}=a_{2} d_{1,2}^{-1} e_{1} a_{1} a_{2} e_{1} a_{1} b_{1} a_{1} e_{1}^{-1} a_{1}^{-1} a_{2}^{-1} e_{1}^{-1} d_{1,2} a_{2}^{-1}=($ by $(\mathrm{J})$ and $(5))$

$a_{2}^{2} d_{1,2}^{-1} e_{1} a_{1} b_{1} a_{1}^{-1} e_{1}^{-1} d_{1,2}=($ by $(\mathrm{J}))$

$a_{2}^{2} d_{1,2}^{-1} b_{1}^{-1} a_{1}^{-1} e_{1} a_{1} b_{1} d_{1,2}$.

The last expression commutes with $a_{1}$ and $b_{1}$, by (J) and (5)).

$$
g_{6}=\left(s a_{1}^{2} t_{1}\right)^{4}
$$

Proof of (30) By the definition $g_{6}=\left(r a_{1} t_{1}\right)^{5}$. The required relation is proved by a rather long computation, using $(\mathrm{J})$. Observe first that $s a_{1}^{2}=\left(b_{1} a_{1}\right)^{3}$, and that $r a_{1}=\left(b_{1} a_{1}\right)^{2}$. We also have

$$
\begin{aligned}
& t_{1}\left(b_{1} a_{1}\right)^{2} t_{1}=e_{1} a_{1} a_{2} e_{1} b_{1} a_{1} b_{1} a_{1} e_{1} a_{1} a_{2} e_{1}= \\
& e_{1} a_{1} b_{1} a_{2} e_{1} a_{1} b_{1} a_{1} e_{1} a_{1} a_{2} e_{1}=b_{1} e_{1} a_{1} b_{1} a_{2} e_{1} a_{1} b_{1} a_{1} e_{1} a_{2} e_{1}= \\
& b_{1} a_{1} e_{1} a_{1} b_{1} a_{2} e_{1} a_{1} b_{1} a_{1} e_{1} a_{2}=b_{1} a_{1} e_{1} a_{1} a_{2} e_{1} b_{1} a_{1} b_{1} a_{1} e_{1} a_{2}= \\
& \left(b_{1} a_{1}\right) t_{1}\left(b_{1} a_{1}\right)^{2} e_{1} a_{2} \\
& \text { and } \\
& e_{1} a_{2}\left(b_{1} a_{1}\right)^{2} t_{1}=b_{1} e_{1} a_{1} a_{2} b_{1} a_{1} e_{1} a_{1} a_{2} e_{1}=b_{1} e_{1} a_{1} a_{2} e_{1} b_{1} a_{1} e_{1} a_{2} e_{1}= \\
& b_{1} e_{1} a_{1} a_{2} e_{1} b_{1} a_{2} a_{1} e_{1} a_{2}=b_{1} a_{1} e_{1} a_{1} a_{2} e_{1} b_{1} a_{1} e_{1} a_{2}=\left(b_{1} a_{1}\right) t_{1}\left(b_{1} a_{1}\right) e_{1} a_{2}= \\
& b_{1} a_{1} e_{1} a_{1} b_{1} a_{2} e_{1} a_{1} e_{1} a_{2}=b_{1} a_{1} b_{1} e_{1} a_{1} b_{1} a_{2} e_{1} a_{1} a_{2}=b_{1} a_{1} b_{1} a_{1} e_{1} a_{1} b_{1} a_{2} e_{1} a_{1}= \\
& \left(b_{1} a_{1}\right)^{2} t_{1}\left(b_{1} a_{1}\right) .
\end{aligned}
$$

The required result follows from the above relations.

This concludes the proof of Theorem 1 .

\section{Mapping class group of a closed surface}

We shall consider in this section the mapping class group $\mathcal{M}_{g}$ of a closed surface $S_{g, 0}$ of genus $g>1$. We shall keep the notation from the previous section. In particular $\mathcal{M}_{g, 1}$ is the mapping class group of $S=S_{g, 1}$ and $S_{g, 0}$ is obtained from $S$ by capping the boundary $\partial$ of $S$ by a disk $D$ with a distinguished center $p$, and then forgetting $p$. We have two exact sequences

$$
1 \rightarrow \mathbb{Z} \stackrel{\psi}{\longrightarrow} \mathcal{M}_{g, 1} \stackrel{\phi}{\longrightarrow} \mathcal{M}_{g, 0,1} \rightarrow 1
$$

\section{Geometry and Topology, Volume 3 (1999)}




$$
1 \rightarrow \pi_{1}\left(S_{g, 0,1}, p\right) \stackrel{\sigma}{\longrightarrow} \mathcal{M}_{g, 0,1} \stackrel{e_{*}}{\longrightarrow} \mathcal{M}_{g, 0,0} \rightarrow 1
$$

In the first sequence the Dehn twist $\Delta=T_{\partial}$ belongs to the kernel of $\phi$. We shall prove now that it generates the kernel. When we split the surface $S_{g, 1}$ open along the curves $\beta_{1}, \alpha_{1}, \epsilon_{1}, \alpha_{2}, \ldots, \epsilon_{g-1}, \alpha_{g}$ we get an annulus $N$, and one boundary of $N$ is equal to $\partial$. If $h \in \operatorname{ker}(\phi)$ than $h$ takes each curve $\gamma \in\left\{\beta_{1}, \alpha_{1}, \epsilon_{1}, \alpha_{2}, \ldots, \epsilon_{g-1}, \alpha_{g}\right\}$ onto a curve $h(\gamma)$ which is isotopic to $\gamma$ in $S_{g, 0}$ by an isotopy fixed on $p$. Therefore $\gamma$ and $h(\gamma)$ form 2-gons which are disjoint from $p$ and hence from $D$. It follows that $h$ is isotopic in $S_{g, 1}$ to a homeomorphism equal to the identity on all curves $\beta_{1}, \alpha_{1}, \epsilon_{1}, \alpha_{2}, \ldots, \epsilon_{g-1}, \alpha_{g}$. But then it is a homeomorphism of the annulus $N$ so it is isotopic to a power of $\Delta$.

The second sequence is described in [2], Theorem 4.3. The kernel of $e_{*}$ is generated by spin-maps $T_{\gamma^{\prime}} T_{\gamma}^{-1}$, where $\gamma$ and $\gamma^{\prime}$ are simple, nonseparating curves which bound an annulus on $S_{g, 0,1}$ containing the distinguished point $p$. The composition $e_{*} \phi$ is an epimorphism from the group $\mathcal{M}_{g, 1}$ onto the group $\mathcal{M}_{g}=\mathcal{M}_{g, 0,0}$ and its kernel is generated by $\Delta$ and by the spin maps $T_{\gamma^{\prime}} T_{\gamma}^{-1}$, where $\gamma$ and $\gamma^{\prime}$ are simple, nonseparating curves on $S$ which bound an annulus with a hole bounded by $\partial$. Clearly all such annuli are equivalent by a homeomorphism of $S$, hence all spin maps are conjugate in $\mathcal{M}_{g, 1}$. It suffices to consider one spin map $T_{\delta_{g}^{\prime}} T_{\delta_{g}}^{-1}$, where $\delta_{g}$ and $\delta_{g}^{\prime}$ are curves on Figure 18. $T_{\delta_{g}}$ is equal to the element $d_{g}$ in the relation (M4).

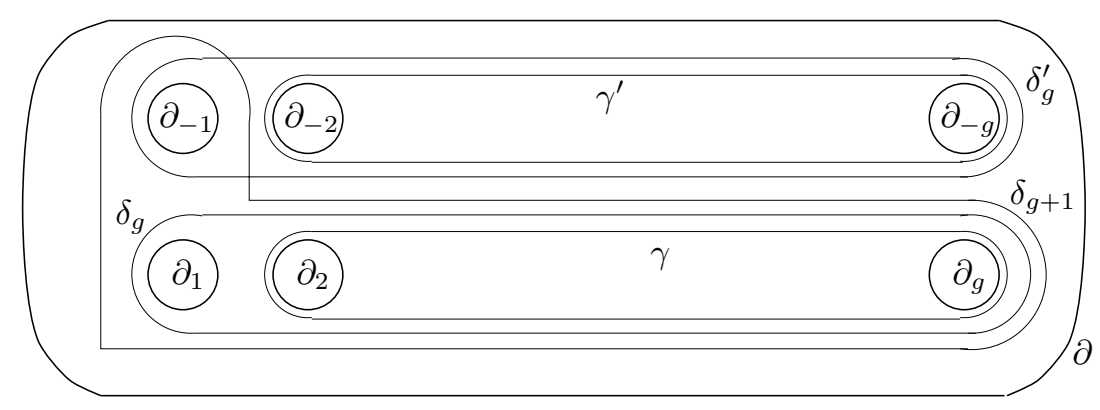

Figure 18: Spin maps in the proof of Theorem 3

Let $w=b_{1} a_{1} e_{1} a_{2} \ldots a_{g-1} e_{g-1} a_{g}^{2} e_{g-1} a_{g-1} \ldots a_{2} e_{1} a_{1} b_{1}$. It is easy to check, drawing pictures, that $w\left(\delta_{g}\right)=\delta_{g}^{\prime}$. Therefore, by Lemma 20, relation (M4) is equivalent, modulo relations in $\mathcal{M}_{g, 1}$, to $T_{\delta_{g}}=T_{\delta_{g}^{\prime}}$. By the above argument $\mathcal{M}_{g}$ has a presentation with relations $(\mathrm{M} 1)-(\mathrm{M} 4)$ and relation $\Delta=1$. We have to prove that the last relation follows from the others.

Let $\mathcal{M}^{\prime}=\left(\mathcal{M}_{g, 1}\right) /(M 4)$. Let $d_{g}, d_{g}^{\prime}, d_{g+1}, c, c^{\prime}$ be twists along curves $\delta_{g}$, 
$\delta_{g}^{\prime}, \delta_{g+1}, \gamma, \gamma^{\prime}$ respectively, depicted on Figure 18. Each element of $\mathcal{M}_{g, 1}$ represents an element in $\mathcal{M}^{\prime}$ which we denote by the same symbol.

From now on, till the end of this section, symbols denote elements in $\mathcal{M}^{\prime}$. We want to prove that $\Delta=1$.

All relations from Lemma 21 are true in $\mathcal{M}^{\prime}$. We have $d_{g} * b_{1}=b_{1}^{-1} * d_{g}$ and $d_{g}$ commutes with every $a_{i}$ and $e_{i}$. By Lemma 21, (iii) and (v) we have:

$\Delta=\left(a_{g} e_{g-1} a_{g-1} \ldots e_{1} a_{1} b_{1} d_{g}\right)^{2 g+2}=$

$\left(a_{g} e_{g-1} a_{g-1} \ldots e_{1} a_{1}\right)^{2 g}\left(b_{1} a_{1} \ldots a_{g} a_{g} \ldots a_{1} b_{1}\right)\left(d_{g} b_{1} a_{1} \ldots a_{g} a_{g} \ldots a_{1} b_{1} d_{g}\right)$,

$d_{g} d_{g}^{\prime}=\left(a_{g} e_{g-1} a_{g-1} \ldots e_{1} a_{1}\right)^{2 g}=$

$\left(a_{g} e_{g-1} a_{g-1} \ldots e_{2} a_{2}\right)^{2 g-2}\left(e_{1} a_{2} \ldots a_{g} a_{g} \ldots a_{2} e_{1}\right)\left(a_{1} e_{1} a_{2} \ldots a_{g} a_{g} \ldots a_{2} e_{1} a_{1}\right)$,

$\left(a_{g} e_{g-1} a_{g-1} \ldots e_{2} a_{2}\right)^{2 g-2}=c c^{\prime}$,

$\left(d_{g} b_{1} a_{1}\right)^{4}=c d_{g+1}$.

We also see that $c^{\prime} d_{g+1}^{-1}$ and $d_{g}^{\prime} d_{g}^{-1}$ are spin maps, hence $c^{\prime}=d_{g+1}$ and $d_{g}=d_{g}^{\prime}$. Therefore

$\left(a_{g} e_{g-1} a_{g-1} \ldots e_{2} a_{2}\right)^{2 g-2}=\left(d_{g} b_{1} a_{1}\right)^{4}$,

$d_{g}^{2}=\left(a_{g} e_{g-1} a_{g-1} \ldots e_{2} a_{2}\right)^{2 g-2}\left(e_{1} a_{2} \ldots a_{g} a_{g} \ldots a_{2} e_{1}\right)\left(a_{1} e_{1} a_{2} \ldots a_{g} a_{g} \ldots a_{2} e_{1} a_{1}\right)$,

hence

$\left(a_{1} e_{1} a_{2} \ldots a_{g} a_{g} \ldots a_{2} e_{1} a_{1}\right)=\left(e_{1} a_{2} \ldots a_{g} a_{g} \ldots a_{2} e_{1}\right)^{-1}\left(d_{g} b_{1} a_{1}\right)^{-4} d_{g}^{2}$.

Further

$\Delta=\left(a_{g} e_{g-1} a_{g-1} \ldots e_{1} a_{1}\right)^{2 g}\left(b_{1} a_{1} \ldots a_{g} a_{g} \ldots a_{1} b_{1}\right)\left(d_{g} b_{1} a_{1} \ldots a_{g} a_{g} \ldots a_{1} b_{1} d_{g}\right)=$ $d_{g}^{2} b_{1}\left(a_{1} e_{1} a_{2} \ldots a_{g} a_{g} \ldots a_{2} e_{1} a_{1}\right) b_{1}\left(d_{g} b_{1} a_{1} \ldots a_{g} a_{g} \ldots a_{1} b_{1} d_{g}\right)=$ $d_{g}^{2} b_{1}\left(e_{1} a_{2} \ldots a_{g} a_{g} \ldots a_{2} e_{1}\right)^{-1}\left(d_{g} b_{1} a_{1}\right)^{-4} d_{g}^{2} b_{1}\left(d_{g} b_{1} a_{1} \ldots a_{g} a_{g} \ldots a_{1} b_{1} d_{g}\right)$.

Now $\left(d_{g} b_{1} a_{1} \ldots a_{g} a_{g} \ldots a_{1} b_{1} d_{g}\right)$ commutes with $d_{g}$, by (M4), and commutes with $b_{1}$ and $a_{1}$, by $(\mathrm{J})$, hence

$\Delta=$

$d_{g}^{2} b_{1}\left(e_{1} a_{2} \ldots a_{g} a_{g} \ldots a_{2} e_{1}\right)^{-1}\left(d_{g} b_{1} a_{1}\right)^{-1}\left(d_{g} b_{1} a_{1} \ldots a_{g} a_{g} \ldots a_{1} b_{1} d_{g}\right)\left(d_{g} b_{1} a_{1}\right)^{-3} d_{g}^{2} b_{1}$

$=d_{g}^{2} b_{1} a_{1} b_{1} d_{g}\left(a_{1}^{-1} b_{1}^{-1} d_{g}^{-1}\right)^{3} d_{g}^{2} b_{1}=1$, by $(\mathrm{J})$.

This concludes the proof of Theorem 3 .

Geometry and Topology, Volume 3 (1999) 


\section{Equivalence of presentations}

In this section we shall prove that the presentations of $\mathcal{M}_{g, 1}$ in Theorems 1 and $1^{\prime}$ are equivalent. The relations (M1) coincide with the relations (A). It follows from relations (A) that $b_{2}$ commutes with the left hand side of the relation (B). Thus (B) is equivalent to

$\left(b_{2} a_{2} e_{1} a_{1} b_{1}^{2} a_{1} e_{1} a_{2} b_{2}\right)\left(a_{2} e_{1} a_{1} b_{1}^{2} a_{1} e_{1} a_{2}\right)^{-1}=\left(b_{1} a_{1} e_{1}\right)^{4}$.

Multiplying by $\left(a_{2} e_{1} a_{1} b_{1}^{2} a_{1} e_{1} a_{2}\right)$ on the right we get

$\left(b_{2} a_{2} e_{1} a_{1} b_{1}^{2} a_{1} e_{1} a_{2} b_{2}\right)=\left(b_{1} a_{1} e_{1}\right)^{4}\left(a_{2} e_{1} a_{1} b_{1}^{2} a_{1} e_{1} a_{2}\right)=\left(b_{1} a_{1} e_{1} a_{2}\right)^{5}$, as in the proof of Lemma $21(\mathrm{v})$, and we get a relation identical with (M2).

We now pass to relation (M3). We shall transform it using relations (M1) and (M2) and then we shall conjugate it by $w=a_{3} e_{2} a_{2} e_{1} a_{1} b_{1}$ to get the relation $(\mathrm{C})$. Since $(\mathrm{M} 1)=(\mathrm{A})$ and $(\mathrm{M} 2)$ is equivalent to $(\mathrm{B})$ in presence of $(\mathrm{M} 1)$, it will prove that (M3) is equivalent to (C) in presence of (M1) and (M2). It follows from (M1) and the definitions that each factor on the right hand side of (M3) commutes with $a_{1} a_{2} a_{3}$, therefore $d_{3}$ also commutes with $a_{1} a_{2} a_{3}$. Recall the relations (5), (7) and (19) from section 4, which follow from the relations (M1) and (M2).

(5) $d_{1,2} t_{1}=t_{1} d_{1,2}$,

(7) $t_{1} t_{2} t_{1}=t_{2} t_{1} t_{2}$

(19) $t_{2} d_{1,2} t_{2} d_{1,2}=d_{1,2} t_{2} d_{1,2} t_{2}$.

We now have

$d_{1,2} d_{1,3} d_{2,3}=d_{1,2} t_{2} d_{1,2} t_{2}^{-1} t_{1} t_{2} d_{1,2} t_{2}^{-1} t_{1}^{-1}=($ by 7$)$

$d_{1,2} t_{2} d_{1,2} t_{1} t_{2} t_{1}^{-1} d_{1,2} t_{2}^{-1} t_{1}^{-1}=($ by 5$) d_{1,2} t_{2} t_{1} d_{1,2} t_{2} d_{1,2} t_{1}^{-1} t_{2}^{-1} t_{1}^{-1}=($ by 7$)$

$d_{1,2} t_{2} t_{1} d_{1,2} t_{2} d_{1,2} t_{2}^{-1} t_{1}^{-1} t_{2}^{-1}=\left(\right.$ by 19) $d_{1,2} t_{2} t_{1} t_{2}^{-1} d_{1,2} t_{2} d_{1,2} t_{1}^{-1} t_{2}^{-1}=($ by 7 and 5$)$

$t_{1}^{-1} d_{1,2} t_{2} t_{1} d_{1,2} t_{2} d_{1,2} t_{1}^{-1} t_{2}^{-1}=($ by 5$) t_{1}^{-1} d_{1,2} t_{2} d_{1,2} t_{1} t_{2} t_{1}^{-1} d_{1,2} t_{2}^{-1}=($ by 7 and 19$)$

$t_{1}^{-1} t_{2}^{-1} d_{1,2} t_{2} d_{1,2} t_{1} t_{2} d_{1,2} t_{2}^{-1}=$ (by 5 and 19) $t_{1}^{-1} t_{2}^{-1} d_{1,2} t_{2} t_{1} t_{2}^{-1} d_{1,2} t_{2} d_{1,2}$.

We now conjugate everything by $w$ and, using (M1), we get

$w * a_{1}=b_{1}, \quad w * e_{1}=a_{1}, \quad w * a_{2}=e_{1}, \quad w * e_{2}=a_{2}, \quad w * a_{3}=e_{2}$,

$w * t_{1}=a_{1} b_{1} e_{1} a_{1}=\tilde{t}_{1}, \quad w * t_{2}=a_{2} e_{2} e_{1} a_{2}=\tilde{t}_{2}, \quad w * d_{1,2}=b_{2}$.

Therefore after conjugation by $w$ the right hand side of (M3) becomes the right hand side of $(\mathrm{C})$. 
We have shown in the proof of relations (20), Case 1c, using only relations (M1), that

$d_{3}=\left(b_{1}^{-1}\left(\left(a_{2} e_{1} e_{2} a_{2} a_{3} e_{2}\right)^{-1} * b_{2}\right) b_{1}^{-1} a_{1}^{-1} e_{1}^{-1} a_{2}^{-1}\right) * b_{2}$.

When we conjugate the last expression by $w$ we get exactly the expression for $\tilde{d}_{3}$ in Theorem $1^{\prime}$.

This proves the equivalence of the presentations in Theorems 1 and $1^{\prime}$.

In order to compare Theorems 3 and $3^{\prime}$ we need another set of generators. Let us call the curves $\beta_{2}, \beta_{1}, \alpha_{1}, \epsilon_{1}, \alpha_{2} \ldots, \epsilon_{g-1}, \alpha_{g}$ - the generating curves. Let $\beta_{g}$ be the curve shown on Figure 1 and let $\beta_{2}^{\prime}$ be a curve which intersects $\epsilon_{g-2}$ once and intersects $\beta_{g}$ once and is disjoint from the other generating curves. Then the curves $\beta_{2}^{\prime}, \alpha_{g}, \epsilon_{g-1}, \alpha_{g-1}, \ldots, \epsilon_{1}, \alpha_{1}, \beta_{1}$ have the same intersection pattern as the generating curves and the curve $\beta_{g}$ plays the same role with respect to these curves as the curve $\delta_{g}$ with respect to the generating curves. Let $b_{g}$ and $b_{2}^{\prime}$ be twists along the curves $\beta_{g}$ and $\beta_{2}^{\prime}$ respectively. Then, by Theorem 1, we have a new presentation of $\mathcal{M}_{g, 1}$ with generators $b_{2}^{\prime}, a_{g}, e_{g-1}, a_{g-1}, \ldots, a_{1}, b_{1}$ and with defining relations (M1'), (M2'), (M3') corresponding to (M1), (M2), (M3). It is a presentation of the same group and therefore, when we express $b_{2}^{\prime}$ in terms of the generators from Theorem 1 , it is equivalent to the presentation ((M1), (M2), (M3)) and to the presentation ((A), (B), (C)). By Theorem 3 the group $\mathcal{M}_{g, 0}$ has a presentation with relations $\left(\mathrm{M}^{\prime}\right),\left(\mathrm{M} 2^{\prime}\right),\left(\mathrm{M}^{\prime}\right)$ and one more relation

$\left(\mathrm{M} 4^{\prime}\right) \quad\left[a_{g} e_{g-1} a_{g-1} \ldots e_{1} a_{1} b_{1}^{2} a_{1} e_{1} \ldots a_{g-1} e_{g-1} a_{g}, b_{g}\right]=1$.

Here $b_{g}$ is some product of generators which represents the Dehn twist of $S_{g, 1}$ along the curve $\beta_{g}$. All such products are equivalent modulo relations (M1'), $\left(\mathrm{M} 2^{\prime}\right),\left(\mathrm{M} 3^{\prime}\right)$. Relation (D) of Theorem $3^{\prime}$ has the same form with $b_{g}$ replaced by $\tilde{d}_{g}$. Therefore in order to check that the presentations in Theorems 3 and $3^{\prime}$ are equivalent it suffices to prove that the expression for $\tilde{d}_{g}$ in (D) also represents the Dehn twist with respect to the curve $\beta_{g}$. This task (of drawing very many pictures) is left to the reader.

\section{References}

[1] J S Birman, H Hilden, On mapping class groups of closed surfaces as covering spaces, from: "Advances in the theory of Riemann surfaces", Ann. Math. Stud. 66 (1971) 81-115

[2] J S Birman, Braids, links and mapping class groups, Annals of Math. Studies $82(1974)$ 
[3] J S Birman, B Wajnryb, Errata: presentations of the mapping class group, Israel J. Math. 88 (1994) 425-427

[4] J Cerf, La stratification naturelle ..., Publ. Math. I.H.E.S. 39 (1970) 5-173

[5] M Dehn, Die Gruppe der Abbildungsklassen, Acta Math. 69 (1938) 135-206

[6] S Gervais, A finite presentation of the mapping class group of an oriented surface, preprint

[7] J Harer, The second homology group of the mapping class group of an orientable surface, Invent. Math. 72 (1983) 221-239

[8] J Hass, P Scott, Intersections of curves on surfaces, Israel J. Math. 51 (1985),90-120.

[9] A Hatcher, W Thurston, A presentation for the mapping class group of a closed orientable surface, Topology, 19 (1980) 221-237

[10] M Heusner, Eine Präsentation der Abbildungsklassengruppe einer geschlossenen, orientierbaren Fläche, Diplom-Arbeit, University of Frankfurt

[11] S Humphries, Generators for the mapping class group, from: "Topology of Low-dimensional Manifolds", LNM 722 (1979) 44-47

[12] D Johnson, Homeomorphisms of a surface which act trivially on homology, Proc. Amer. Math. Soc. 75 (1979) 119-125

[13] R C Kirby, A calculus of framed links in $S^{3}$, Invent. Math. 45 (1978) 35-56

[14] T Kohno, Topological invariants for 3-manifolds using representations of mapping class groups I, Topology, 31 (1992) 203-230

[15] F Laudenbach, Presentation du groupe de diffeotopies d'une surface compacte orientable, Travaux de Thurston sur les surfaces, Asterisque 66-67 (1979) 267282

[16] Ning Lu, A simple proof of the fundamental theorem of Kirby calculus on links, Trans. Amer. Math. Soc. 331 (1992) 143-156

[17] M Matsumoto, A presentation of mapping class groups in terms of Artin groups and geometric monodromy of singularities, preprint

[18] S Matveev, M Polyak, On a tangle presentation of the mapping class groups of surfaces, Contemporary Mathematics, 164 (1994) 219-229

[19] J McCool, Some finitely presented subgroups of the automorphism group of a free group, J. Algebra, 35 (1975) 205-213

[20] B Wajnryb, A simple presentation for the mapping class group of an orientable surface, Israel J. Math. 45 (1983) 157-174 